\title{
WestVirginiaUniversity
}

THE RESEARCH REPOSITORY @ WVU

Graduate Theses, Dissertations, and Problem Reports

2016

\section{Safe Semi-Supervised Learning with Sparse Graphs}

Prithish Banerjee

Follow this and additional works at: https://researchrepository.wvu.edu/etd

\section{Recommended Citation}

Banerjee, Prithish, "Safe Semi-Supervised Learning with Sparse Graphs" (2016). Graduate Theses,

Dissertations, and Problem Reports. 5154.

https://researchrepository.wvu.edu/etd/5154

This Dissertation is protected by copyright and/or related rights. It has been brought to you by the The Research Repository @ WVU with permission from the rights-holder(s). You are free to use this Dissertation in any way that is permitted by the copyright and related rights legislation that applies to your use. For other uses you must obtain permission from the rights-holder(s) directly, unless additional rights are indicated by a Creative Commons license in the record and/ or on the work itself. This Dissertation has been accepted for inclusion in WVU Graduate Theses, Dissertations, and Problem Reports collection by an authorized administrator of The Research Repository @ WVU.

For more information, please contact researchrepository@mail.wvu.edu. 


\title{
Safe Semi-Supervised Learning with Sparse Graphs
}

\author{
Prithish Banerjee \\ Dissertation submitted to the \\ Eberly College of Arts and Sciences \\ at West Virginia University \\ in partial fulfillment of the requirements \\ for the degree of \\ Doctor of Philosophy \\ in \\ Computational Statistics \\ Mark V. Culp, Ph.D., Chair \\ Erdogan Gunel, Ph.D. \\ Yanqing Hu, Ph.D. \\ Michael Mays, Ph.D. \\ Kenneth J. Ryan, Ph.D. \\ George A. Spirou, Ph.D. \\ Department of Statistics \\ Morgantown, WV \\ 2016
}

Keywords: Semi-Supervised Learning; Graph-Based Learning

Copyright 2016 Prithish Banerjee 


\section{Abstract \\ Safe Semi-supervised Learning with Sparse Graphs}

\section{Prithish Banerjee}

There has been substantial interest from both computer science and statistics in developing methods for graph-based semi-supervised learning. The attraction to the area involves several challenging applications brought forth from academia and industry where little data are available with training responses while lots of data are available overall. Ample evidence has demonstrated the value of several of these methods on real data applications, but it should be kept in mind that they heavily rely on some smoothness assumptions. The general framework for graph-based semi-supervised learning is to optimize a smooth function over the nodes of the proximity graph constructed from the feature data which is extremely time consuming as the conventional methods for graph construction in general create a dense graph. Lately the interest has shifted to developing faster and more efficient graph-based techniques on larger data, but it comes with a cost of reduced prediction accuracies and small areas of application. The focus of this research is to generate a graph-based semi-supervised model that attains fast convergence without losing its performance and with a larger applicability. The key feature of the semi-supervised model is that it does not fully rely on the smoothness assumptions and performs adequately on real data. Another model is proposed for the case with availability of multiple views. Empirical analysis with real and simulated data showed the competitive performance of the methods against other machine learning algorithms. 


\section{Acknowledgement}

Firstly, I would like to express my sincere gratitude to my advisor Dr. Mark V. Culp for his continuous support throughout my Ph.D. studies and research and for his patience, motivation, and immense knowledge. His guidance helped me during the time of research and writing of this dissertation. I can not imagine having a better advisor and mentor for my Ph.D. studies.

Besides my advisor, I would like to thank the rest of my dissertation committee: Dr. Erdogan Gunel, Dr. Yanqing Hu, Dr. Michael Mays, Dr. Kenneth J. Ryan, and Dr. George A. Spirou, for their insightful suggestions, comments, encouragement, and also the motivations which stimulated me to widen the scope of my research from various perspectives.

My sincere thanks goes to Dr. E. James Harner, for giving me the opportunity to start the journey towards my Ph.D., and also to Dr. Arun Ross, who provided me with an assistantship at a time of my distress. Without their precious support it would not be possible for me to be here today.

I extend some special words of gratitude to my friends, Dibyadyuti, for the fun we have had in the last four years and Michael, for helping me out in so much with my research during the last couple of years.

I thank my wife, Broti, for always being a major source of support even when things would got a bit discouraging. Without her constant care, support, and understanding, this dissertation would not be possible.

Last but not the least, I would like to thank my family for their moral support throughout the dissertation and my life in general.

The material is based upon the work supported by National Science Foundation under Grant no. 1255045. Any opinions, findings, and conclusions or recommendations expressed in this material are those of the author and do not necessarily reflect the view of National Science Foundation. 


\section{Summary}

$\begin{array}{lll}1 \text { Introduction } & 1\end{array}$

1.1 Organization of the Dissertation $\ldots \ldots \ldots \ldots$

\begin{tabular}{|lll|}
\hline 2 & Graph-Based Semi-Supervised Learning with BIG Data & 7
\end{tabular}

2.1 Statistical Machine Learning Problem Setup . . . . . . . . . . . . . 7

2.2 An Overview of Semi-Supervised Learning . . . . . . . . . . . . . . 12

$2.2 .1 \quad$ Proximity Graph Construction $\ldots \ldots \ldots \ldots \ldots$

2.2 .2 Semi-Supervised Optimization . . . . . . . . . . . . . . . 17

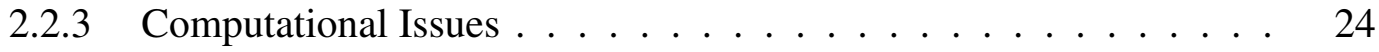

2.3 Anchor Graphs in Semi-Supervised Learning $\ldots \ldots \ldots \ldots$

2.3 .1 Anchor Points . . . . . . . . . . . . . . . . 27

2.3 .2 Local Anchor Embedding $\ldots \ldots \ldots$. . . . . . . . . 30

$2.3 .3 \quad$ Graph Design $\ldots \ldots \ldots \ldots \ldots \ldots \ldots$

$2.3 .4 \quad$ Regression and Classification with Anchor Graphs $\ldots . . . . . .37$

2.4 Empirical Demonstration . . . . . . . . . . . . . . . . . . . . . . . . . . . 39

2.5 Discussion . . . . . . . . . . . . . . . . . . . . . . . . . 42

3 On Safe Semi-Supervised Learning 45

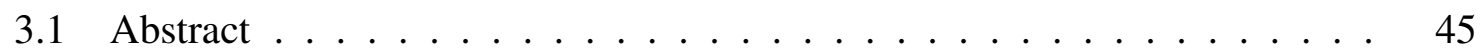

3.2 Introduction $\ldots \ldots \ldots \ldots \ldots \ldots$ 
3.3 Safe Semi-Supervised Prediction . . . . . . . . . . . . . . . . . . 48

3.3.1 The Cluster Assumption Applied to Residuals . . . . . . . . . . . 52

3.3 .2 Existence and Uniqueness $\ldots \ldots \ldots \ldots \ldots$

3.4 Joint Semi-Parametric Prediction . . . . . . . . . . . . . . . . . . . . . 56

$3.4 .1 \quad$ Joint Laplacian and Joint Spreading Derivatives . . . . . . . . . 58

3.5 Results . . . . . . . . . . . . . . . . . . . . . . . . . . . . 61

3.5 .1 Semi-Supervised Smoothness in Regression . . . . . . . . . . . . 62

3.5 .2 Real Comparisons $\ldots \ldots \ldots \ldots \ldots$

3.6 Discussion $\ldots \ldots \ldots \ldots \ldots \ldots$

\begin{tabular}{|lll}
\hline 4 & On Multi-View Learning with Sparse Graphs & 68
\end{tabular}

$4.1 \quad$ Abstract $\ldots \ldots \ldots \ldots \ldots$

4.2 Introduction $\ldots \ldots \ldots$

4.3 Semi-Supervised Shrinking $\ldots \ldots \ldots$. . . . . . . . . . . . . . . . 72

4.4 Joint Multi-View Learning Framework $\ldots \ldots \ldots$

$4.5 \quad$ Joint Training with Sparse Graphs $\ldots \ldots \ldots \ldots \ldots \ldots$

$4.5 .1 \quad$ Multi-View Learning Bound $\ldots \ldots \ldots \ldots$

4.6 Empirical Performance $\ldots \ldots \ldots \ldots$

4.6 .1 Simulation $\ldots \ldots \ldots \ldots \ldots \ldots \ldots \ldots \ldots \ldots$

4.6 .2 Real Data . . . . . . . . . . . . . . . . . . . . 82

4.7 Discussion $\ldots \ldots \ldots \ldots \ldots \ldots \ldots$

5 Discussion and Future Directions $\quad 88$

$5.1 \quad$ General Discussion $\ldots \ldots \ldots \ldots \ldots$. . . . . . . . . . . 88

5.2 Future Research Directions . . . . . . . . . . . . . . . . . . . . . . . . . . . 89

\begin{tabular}{|ll|}
\hline A Investigation of Tuning Parameters & 91
\end{tabular} 
A.1 Investigation on the Anchor Points . . . . . . . . . . . . . . . . 91

A.2 Investigation on the Number of Anchors 's $\ldots \ldots \ldots 2$

A.3 Investigation on the LAE Threshold ${ }^{\prime} c_{n}{ }^{\prime} \ldots \ldots \ldots \ldots$ 


\section{List of Figures}

2.1 (a) Swiss Roll Data Set in Feature Space with Response Regions in a 3D Lattice. (b) Sparse Proximity Graph Approximation of the Swiss Roll. . . . 16

2.2 A 2D Rat Maze Data Set with $n=487,|L|=2$, and Binary Gray or Black Responses Satisfying the Cluster Assumption. . . . . . . . . . . . . . . . . 17

$2.3 \quad$ A Two Moons Example with $|L|=6$ Labeled Cases and $|U|=994$ Unlabeled Cases. The Moon Determines the True Binary Classification (Black or Gray). 27

\begin{tabular}{|ll|l|l|l}
\hline 2.4 & Two Moons from Figure & 2.3 & Revisited. (a) Labeled Cases as Anchors $m=$ \\
\hline
\end{tabular} $|L|=6$. (b) Randomly Selected Rows of $\boldsymbol{X}$ as Anchors $m=150$. . . . . . . 28

\begin{tabular}{|ll|l|l|l|l|l|l|l|}
\hline 2.5 & Two Moons from Figures & 2.3 & and & 2.4 & Revisited. Anchors are the $m=150$
\end{tabular} Centroids from (a) $k$-Means and (b) Hierarchical Clustering. . . . . . . . . 29

\begin{tabular}{|ll|l|l|l|l|}
\hline 2.6 & Two Moons from Figures & 2.3 & 2.5 & Revisited. (a) Anchor Graph Edges Be-
\end{tabular} tween Anchors $m=150$ and Observations $n=1,000$. (b) Graph $W$ Edges Between Observations. . . . . . . . . . . . . . . . . 36

\begin{tabular}{|ll||l|l|}
\hline 2.7 & Two Moons from Figures & 2.3 & Revisited. Prediction Borders Under Lo-
\end{tabular} gistic Loss with (a) the Anchor Graph Optimization (2.25) and (b) Labeled Loss Optimization (2.15). . . . . . . . . . . . . . . . . . . . . . 39

2.8 Performance (Left) and Time (Right) for Real Data Set Benchmarks. (1) $k$ NN with Laplacian, (2) $k$-NN with Normalized Laplacian, and (3) Anchor Graph. . . . . . . . . . . . . . . . . 
3.1 Simulation Results for the "Two Moons" Regression Example. (a) A 3D

Plot of the True Response Surface with its 2D Shadow on the Feature Space. Unlabeled RMSE: (b) $\mu=0$ and (c) $\mu=-5$. . . . . . . . . . . . . . . . 60

3.2 Unlabeled Performance on Publicly Available Data Sets: Regression (top two rows) and Classification (bottom row). The labeled sets were selected at random with labeled percentages $100|L| / n=10,15,20,25,30,50 \%$. Performance measures are unlabeled RMSE in the regression examples and unlabeled error rate in the classification examples. . . . . . . . . . . . . 63

4.1 Semi-Supervised Shrinking Examples. . . . . . . . . . . . . 71

4.2 Simulated Two Moons Example. . . . . . . . . . . . . . . . 81

4.3 Unlabeled Performance on Real Data Sets. . . . . . . . . . . . . . . . . . 83 


\section{Chapter 1}

\section{Introduction}

Automation and learning in the era of "Big Data" are the cornerstone of modern machine learning methods. The main idea is to predict new data points given a sequence of 'training' points. In many cases, these approaches are viewed as adapting to the prediction problem at hand, often emphasizing certain features within the training points while down weighting other less meaningful features of these points. This is all done on-the-fly in real time. This ability is often viewed as a learning paradigm and has deep roots within statistics and computer science (Hastie et al. 2009). In order to do this task, one must have methods that (i) are computationally efficient, (ii) can learn all the parameters quickly in real time on the training points, and (iii) are well grounded in theory. Machine learning is the field attributed to providing data driven algorithms and models for exploring the data to make these predictions on real applications. Machine learning approaches tend to show promise in several practical applications including those listed below.

Cybernetics and System Science: Artificial intelligence (AI) and machine learning are some of modern research methods used in the field of cybernetics and system science. Automated biometrics recognition systems provide a clear example of how machine learning methods paired with AI help advance this important field. The goal 
is to uniquely identify a person in a fully automated fashion based on their biometric traits such as fingerprint, iris, and facial image match scores or other biometric modalities (Jain et al., 2004). In movies, such identification of the suspect is usually shown instantaneously, but this task in reality is daunting primarily due to the quality and sheer volume of the biometric data that must be processed in order to form a match. Calibrating uncertainty of matches and providing probabilistic feedback in real time on big data are a direct application of machine learning and are already having a profound practical impact on this field (Kung et al., 2005; Palaniappan and Mandic, 2007).

Speech recognition: This problem involves identification of certain dialects and languages for communication. The data typically consists of different speech recordings that are quantified into a matrix with aid from linguistics experts (Deng et al., 2013).

Text categorization: Filtering out spam emails, categorizing user messages, and recommending internet articles are some of the tasks that one hopes computationally efficient algorithm can solve (Sebastiani, 2002). Another pertinent and seemingly simpler problem is that of determining whether or not a text message is 'interesting.' Individuals cannot manually perform this relevant task in real time given the volume of information available at a given time point, so the machine learning has gained traction in this content area.

Neuroscience: Mapping out the network of dendrons, exons, and cell bodies is a nontrivial and time consuming process (Lao et al., 2004, Richiardi et al., 2013), but is necessary to better understand the functioning of the brain. Machine learning approaches have had a significant impact on this challenging and practical problem.

The focus of this dissertation is on semi-supervised learning from a machine learning point-of-view with graphs. Semi-supervised learning in general is widely regarded as a 
compromise between unsupervised and supervised learning. Elements of these two extreme learning paradigms are summarized below.

Unsupervised Learning: Suppose a $n \times p$ data set, $\boldsymbol{X}$, is generated in some application. Each row of $\boldsymbol{X}$ is an observation while each column is a variable. For example, the rows often represent different text documents in text categorization, and a column represents some common numerical summary, e.g., the number of times a keyword appears in a document. The goal in unsupervised learning is to hunt for patterns within the data that are informative about the application domain. In the text example, the documents could be papers about climate change that are published in a well-known journal, and the researcher's goal may be to determine what word frequencies scientists use most often to describe the current-state of climate change. Different methods tend to dig for patterns within the data and usually involve some form of clustering (Tryon, 1939; Everitt and Hothorn, 2011), ranking (Page et al., 1999), or dimensionality reduction (Pudil and Novovičová, 1998).

Supervised Learning: An $n \times p$ data matrix $\boldsymbol{X}$ may still be collected, but unlike unsupervised learning, an $n \times 1$ response vector $y$ is also observed. In this case, the data come in pairs $\left(\boldsymbol{x}_{1}, y_{1}\right), \cdots,\left(\boldsymbol{x}_{n}, y_{n}\right)$, where vector $\boldsymbol{x}_{i} \in \mathbb{R}^{p}$ is stored as the $i$ th row of $\boldsymbol{X}$ for $i=1, \ldots, n$. The goal is to determine how $\boldsymbol{X}$ can be used to predict, describe, or make inferences about the response vector $y$. Performance metrics are introduced to measure how well one can assess the prediction of the response $y_{0}$ for a new data point $\boldsymbol{x}_{0}$. An easy-to-understand example comes from text categorization. Suppose one wishes to identify whether or not an email is 'spam.' The response is the email type, i.e., 'spam' and 'not spam,' and the goal would be to train and apply a learner to categorize emails as they arrive in real time. This spam application is an example of classification, where the response $y_{0}$ takes values from a finite discrete set. On the 
other hand, if $y_{0} \in \mathbb{R}$ or $\boldsymbol{y}_{0} \in \mathbb{R}^{d}$, then the problem is a regression application.

Similar to supervised learning, in semi-supervised learning, we assume that a $n \times p$ fea-

ture data set $\boldsymbol{X}$ and response vector $\boldsymbol{y}$ are observed, but unlike in supervised learning the responses are observed for only a proper subset of the observations $L \subseteq\{1, \cdots, n\}$ (labeled set) and missing for the remaining observations $U=\{1, \cdots, n\}-L$ (unlabeled set). This problem is partially unsupervised in that many rows of $\boldsymbol{X}$ will have no available response labeling. The focus of this research is to define a computationally efficient semi-supervised paradigm applicable to large data problems that is also grounded in a comprehensive theory.

\subsection{Organization of the Dissertation}

Chapter 2: This chapter provides the general problem setup, literature reviews, and background necessary for this work. The general machine learning problem is first defined with particular focus on the optimization leading to the enhancements necessary for the semisupervised part of the problem under examination. Proximity graph-based methods are discussed along with computational issues, followed by the anchor graph construction methodology (Liu et al., 2010). This dissertation builds substantially on this prior work. The downside to this prior work is that the resulting classifiers tend to perform poorly (as verified in this work), are not very general, and are typically presented as heuristic methods. The approaches proposed herein dramatically improve the performance on real and simulated data, are much more general, and are grounded in a comprehensive learning theory when compared to the prior work.

Chapter 3: A novel state-of-the-art fast method known as the safe semi-supervised semiparametric model (S4PM) is proposed. The S4PM promotes a compromise between 'pure' semi-supervised and 'pure' supervised estimation in a fairly innovative and novel way. In doing this, we demonstrate that our approach routinely is among one of the top competi- 
tors for supervised and nearly always for semi-supervised. Concrete explanations for why performance improvements are expected are theoretically demonstrated in this work. The dominance of the S4PM approach is empirical validated especially in the regression setting. This is intuitive since regression is generally a harder problem than classification and knowing where the predictions are in terms of the $\boldsymbol{X}$-space gives the semi-supervised method a clear advantage over supervised. The technique also has some clear robustness to noise in the responses, the feature data, or both. This type of presentation for the value of unlabeled data in training is contrary to explanations in the existing literature, since (i) nearly all existing empirical demonstrations offered in the literature are in the classification setting and the regression setting is rarely considered; (ii) nearly all accepted theoretical explanations are provided with either the cluster assumption, manifold assumption, or various smoothness assumptions which are almost always based on classification problems with no noise; and (iii) computational efficiencies with existing semi-supervised work are rarely considered.

Chapter 4: In this chapter an innovative multi-view learning approach that builds on our foundation in Chapter 2 and our safe work in Chapter 3 is proposed. Mathematically, the observations are assumed to have a manifold embedded within particular subsets of the variables. It is not clear whether capturing such manifolds yields performance improvements, so several mechanisms are carefully introduced to trade-off situations where identification of the manifold are useful versus circumstances where knowing the manifolds is of little value. Essentially, the framework is a double partitioning. The variables have a class membership defined by known views as part of the data generation process, and within each view the observations have a class membership corresponding to certain unknown manifold structures. The ultimate result of this work is an empirical multi-view semi-supervised learning technique that is both computationally efficient and performs strongly on real data.

Chapter 5: Some important concluding remarks are drawn along with some interesting future directions of this research in the final chapter. 
Appendix 1: Not much is known about the effect of the tuning parameters of the anchor graph approach on practical problems. This appendix provides some interesting tests to help understand the effects of tuning parameters on challenging real datasets. 


\section{Chapter 2}

\section{Graph-Based Semi-Supervised Learning with BIG Data}

Prithish BanerJee, Mark Vere Culp, Kenneth Joseph Ryan and George

MiCHAILIDIS

Submitted as Chapter in the Handbook of Research on Applied Cybernetics and Systems Science

\subsection{Statistical Machine Learning Problem Setup}

Statistical machine learning addresses the issue of predicting new data points given a training set of data points. Let $y_{i} \in \mathbb{R}$ and $x_{i} \in \mathbb{R}^{p}$ be the response and feature vector for observation $i=1, \ldots, n$. The response vector is $\boldsymbol{y}=\left(y_{1}, \cdots, y_{n}\right)^{T}$, and the $n \times p$ matrix of feature data $\boldsymbol{X}$ simply stacks the $\boldsymbol{x}_{1}, \ldots, \boldsymbol{x}_{n}$ as row vectors. The goal in machine learning under this setup is to construct a function $f: \mathbb{R}^{p} \rightarrow \mathbb{R}$ for the prediction of a new $y_{0}$ given its feature 
information $x_{0}$. In order to make this endpoint well-defined, we might first assume that the data $\left(y_{0}, \boldsymbol{x}_{0}\right),\left(y_{1}, \boldsymbol{x}_{1}\right), \ldots,\left(y_{n}, \boldsymbol{x}_{n}\right)$ are independent and identically distributed from some measurable joint distribution $P$, and this assumption is made throughout this chapter for ease of exposition.

The function $f$ can be chosen as the minimizer of some prediction error metric, typically motivated by some justifiable loss function. In a regression context, the squared error loss function $L\left(y_{0}, f\left(\boldsymbol{x}_{0}\right)\right)=\left(y_{0}-f\left(\boldsymbol{x}_{0}\right)\right)^{2}$, which measures the square of the deviation between a response and the function $f$, is widely considered to be the default choice and results in the expected squared error prediction metric

$$
\mathbf{E}\left[\left(y_{0}-f\left(\boldsymbol{x}_{0}\right)\right)^{2}\right]=\int\left(y_{0}-f\left(\boldsymbol{x}_{0}\right)\right)^{2} d P
$$

The squared error loss function $L\left(y_{0}, f\left(\boldsymbol{x}_{0}\right)\right)=\left(y_{0}-f\left(\boldsymbol{x}_{0}\right)\right)^{2}$ measures the deviation between a response and the function. Other loss functions generalize (2.1) and will be considered later in this work. The integral in 2.1 is taken over the unknown response $y_{0}$ and its feature data $\boldsymbol{x}_{0}$. As such, it is common to consider the conditional point-wise minimization problem for constructing a function $f$ from training data $(\boldsymbol{y}, \boldsymbol{X})$, i.e.,

$$
f\left(\boldsymbol{x}_{0}\right)=\underset{a \in \mathbb{R}}{\arg \min } \mathbf{E}\left[\left(y_{0}-a\right)^{2} \mid \boldsymbol{x}_{0}\right]
$$

having solution

$$
f\left(\boldsymbol{x}_{0}\right)=\mathbf{E}\left[\boldsymbol{y} \mid \boldsymbol{x}_{0}\right]
$$

Many approaches produce an estimated function $\widehat{f}$ that approximates 2.2 . The global 
fit of an ordinary least squares (OLS) linear regression

$$
\mathbf{E}\left[\boldsymbol{y} \mid \boldsymbol{x}_{0}\right] \approx \boldsymbol{x}_{0}^{\prime}\left(\boldsymbol{X}^{T} \boldsymbol{X}\right)^{-1} \boldsymbol{X}^{T} \boldsymbol{y}
$$

provides one such approximation, and the local averaging of $k$-Nearest Neighbors $(k-\mathrm{NN})$

$$
\mathbf{E}\left[\boldsymbol{y} \mid \boldsymbol{x}_{0}\right] \approx \operatorname{Ave}\left(y_{i} \mid \boldsymbol{X}_{i} \in \boldsymbol{N}_{k}\left(\boldsymbol{x}_{0}\right)\right)
$$

is another well-known yet different approximation approach, where $\boldsymbol{N}_{k}\left(\boldsymbol{x}_{0}\right) \subset\left\{\boldsymbol{x}_{1}, \ldots, \boldsymbol{x}_{n}\right\}$ such that $\left|\boldsymbol{N}_{k}\left(\boldsymbol{x}_{0}\right)\right|=k$ is the neighborhood of $\boldsymbol{x}_{0}$. Both approaches have a tuning parameter that trades-off the bias and variance of prediction errors. For example, $k$-NN exhibits low bias and high variance with $k=1$ and high bias and low variance with $k=N$, so its tuning parameter $k$ spans the trade-off. In the case of linear regression $(2.3)$, the trade-off is more subtle, but the choice $p$ (the number of columns in $\boldsymbol{X}$ ) does indeed span the bias and variance trade-off. In general, it is sensible to use cross-validation to estimate the values of tuning parameters in order to enhance performance, possibly on an empirical version of the underlying theoretical performance metric. This section lines-up with this use of cross-validation, although rationales for other approaches are found in the literature (Efron, 1983; Bowman, 1984; Browne et al., 1993; Hastie et al., 2009).

For ease of presentation throughout this Chapter, an aggregate loss function

$$
\mathscr{L}(\boldsymbol{y}, \boldsymbol{f})=\sum_{i=1}^{n} L\left(y_{i}, f_{i}\right)
$$

is often assumed, where $L: \mathbb{R} \times \mathbb{R} \rightarrow \mathbb{R}$ is the observation level loss function for predicting response $y_{i}$ with $\boldsymbol{f}_{i}$ for $i=1, \ldots, n$. Many techniques can also be motivated as the solution 
to a penalized optimization problem of the form

$$
\min _{f} \mathscr{L}(\boldsymbol{y}, \boldsymbol{f})+\lambda \mathbb{J}(\boldsymbol{f})
$$

where $\mathscr{L}: \mathbb{R}^{n} \times \mathbb{R}^{n} \rightarrow \mathbb{R}$ is an aggregate loss function $(2.5), \lambda$ is the Lagrangian multiplier referred as smoothing parameter, and $\mathbb{J}(\boldsymbol{f})$ is the penalization function that varies rapidly over small regions. Clearly, OLS linear regression 2.3 follows by assuming $\boldsymbol{f}=\boldsymbol{X} \beta$ with $\beta \in \mathbb{R}^{p}$ and $\lambda=0$. The connection between an optimization 2.6 and $k$-NN is more subtle. but to see it, use the indicator function $\mathscr{I}_{\{\cdot\}}$ to define a two-sided local kernel

$$
K_{k}\left(\boldsymbol{x}_{i}, \boldsymbol{x}_{j}\right)=\mathscr{I}_{\left\{i \in \boldsymbol{N}_{k}(\boldsymbol{x})_{j} \text { or } j \in \boldsymbol{N}_{k}(\boldsymbol{x})_{i}\right\}} \cdot
$$

A one-sided non-symmetric kernel could also be used depending on implementation of $k$ NN. The prediction function at $x_{0}$ then solves

$$
\widehat{f}\left(\boldsymbol{x}_{0}\right)=\underset{a \in \mathbb{R}}{\arg \min } \sum_{i=1}^{n} K_{k}\left(\boldsymbol{x}_{i}, \boldsymbol{x}_{0}\right)\left(y_{i}-a\right)^{2} .
$$

The generic prediction 2.7) can be applied to each training data point $\boldsymbol{x}_{i}$ to get $\widehat{f}\left(\boldsymbol{x}_{i}\right)$ for $i=1, \ldots, n$, so the corresponding $n \times 1$ vector $\widehat{\boldsymbol{f}}=\left(\widehat{f}\left(\boldsymbol{x}_{1}\right), \ldots, \widehat{f}\left(\boldsymbol{x}_{n}\right)\right)^{T}$ solves

$$
\underset{\boldsymbol{f}}{\arg \min }(\boldsymbol{y}-\boldsymbol{f})^{T} \boldsymbol{W}(\boldsymbol{y}-\boldsymbol{f})+\boldsymbol{f}^{T} \Delta \boldsymbol{f}
$$

where $\boldsymbol{W}$ with components $\boldsymbol{W}_{i j}=K_{k}\left(\boldsymbol{x}_{i}, \boldsymbol{x}_{j}\right)$ is the Gram matrix for the kernel and the Laplacian matrix $\boldsymbol{\Delta}=\operatorname{diag}(\boldsymbol{W} \overrightarrow{1})-\boldsymbol{W}=\boldsymbol{D}-\boldsymbol{W}$ defines the penalty term. Thus, $k$-NN is indeed a special case of (2.6). Generalizations of (2.6) are of particular note and elaborated on next.

In the case of the linear regression, it is common to consider more general penalty functions for $\lambda>0$, e.g., Ridge Regression $\mathbb{J}(\boldsymbol{f})=\boldsymbol{\beta}^{\prime} \boldsymbol{\beta}$, Lasso $\mathbb{J}(\boldsymbol{f})=\sum_{j=1}^{p}\left|\beta_{j}\right|$, and Elastic 
Net, a convex combination of ridge and lasso regression penalty terms. OLS linear regression clearly optimizes over the column space $C(\boldsymbol{X})$ of $\boldsymbol{X}$ since $\widehat{\boldsymbol{f}} \in C(\boldsymbol{X})$.

Generalizing away from the column space of $\boldsymbol{X}$ brings another interesting idea. Suppose $\widehat{\boldsymbol{f}}$ has expansion $\widehat{\boldsymbol{f}}(\boldsymbol{x})=\sum_{j=1}^{p} \hat{\beta}_{m} \boldsymbol{h}_{m}(\boldsymbol{x})=\boldsymbol{h}(\boldsymbol{x})^{T} \hat{\boldsymbol{\beta}}$ where $\boldsymbol{h}(\boldsymbol{x})^{T}=\left(\boldsymbol{h}_{1}(\boldsymbol{x}), \cdots, \boldsymbol{h}_{p}(\boldsymbol{x})\right)$ is the set of basis functions. Construction of the basis functions requires the specification of knot points, which can be a tedious task. Thus motivates the idea of smoothing spline,

$$
\widehat{\boldsymbol{f}}_{\lambda}=\underset{\boldsymbol{f}: \boldsymbol{f}^{\prime \prime} \text { exist }}{\arg \min }(\boldsymbol{y}-\boldsymbol{f})^{T}(\boldsymbol{y}-\boldsymbol{f})+\lambda \int\left(\boldsymbol{f}^{\prime \prime}(\boldsymbol{x})\right)^{2} d x
$$

It is evident that $\lambda=0$ leads to OLS i.e. in the $p=1$ case of low bias, high variance and $\lambda=\infty$ produces the opposite extreme of high bias, low variance. The Reinsch form of the smoothing spline

$$
\min _{\boldsymbol{f}}(\boldsymbol{y}-\boldsymbol{f})^{T}(\boldsymbol{y}-\boldsymbol{f})+\lambda \boldsymbol{f}^{T} \boldsymbol{P} \boldsymbol{f}
$$

with some positive semi-definite penalty matrix $\boldsymbol{P}$ is also of note. It is well-known that a smoothing spline can be generalized in terms of a Hilbert space optimization problem where one specifies a Hilbert space consisting of twice differentiable functions $f^{\prime \prime}$ whose $f$ and $f^{\prime}$ are absolutely continuous (Heckman, 1995). This line of thought leads to a more general approach for large $p$ that begins with the representer inner product kernel $\widetilde{K}\left(\boldsymbol{x}_{i}, \boldsymbol{x}_{j}\right)$, the endowed Hilbert space $\mathscr{H}_{\widetilde{K}}$, and the appropriate inner product. This inner product is chosen by construction, so the kernel function is the representer for $\mathscr{H}_{\widetilde{K}}$. The optimization of $f$ is

$$
\min _{\boldsymbol{f} \in \mathscr{H}_{\widetilde{K}}} \mathscr{L}(\boldsymbol{y}, \boldsymbol{f})+\lambda\|\boldsymbol{f}\|_{\mathscr{H}_{\widetilde{K}}}^{2}
$$


and is achieved on finite data with $\widehat{\boldsymbol{f}}=\widetilde{K} \widehat{\alpha}$, where

$$
\widehat{\alpha}=\underset{\alpha \in C(\widetilde{K})}{\arg \min } \mathscr{L}(\boldsymbol{y}, \widetilde{K} \alpha)+\lambda \alpha^{T} \widetilde{K} \alpha
$$

The finite representation $(2.12)$ of $(2.11)$ is a special case of 2.6 and is made possible because of a mathematical theorem that is often referred to in non-technical terms as the 'kernel trick' in the literature. A further generalization of $(2.8)$ is observed when the Gram matrix $\boldsymbol{W}$ is taken from an arbitrary Gaussian kernel

$$
K_{\sigma}\left(\boldsymbol{x}_{i}, \boldsymbol{x}_{j}\right)=\frac{\exp \left(-\left\|\boldsymbol{x}_{i}-\boldsymbol{x}_{j}\right\|^{2}\right)}{2 \sigma^{2}}
$$

Optimizations (2.6), 2.8), 2.10), and (2.11) motivate many popular supervised techniques and are extended to semi-supervised learning next in Subsection 2.2

\subsection{An Overview of Semi-Supervised Learning}

Semi-supervised learning is a class of machine learning approaches with commonalities to supervised and unsupervised learning. It is the extension of supervised learning that involves training with feature data observations where a proper subset of some (or possibly many or pretty much all) of these observations have a missing response. A practical motivation for semi-supervised learning is that acquiring responses may be relatively costly or time consuming, so it may be infeasible to obtain a large number of responses in certain applications. On the other hand, it may be possible to obtain the feature data $\boldsymbol{X}$ in a relatively inexpensive and easy manner. The ultimate goal of this practical machine learning area is to routinely outperform supervised (and sometimes unsupervised) learning approaches in terms of common performance metrics. The probability a response was observed is assumed to be independent of the response and feature data throughout this dissertation. This so-called MCAR or 
Missing Completely at Random assumption is often assumed in semi-supervised learning although it is rarely acknowledged (Lafferty and Wasserman, 2008).

The available data set partitions into two subsets based on whether or not the response was observed for a given observation. The random subset of observations with responses is called the labeled set (i.e., $L=\left\{i: y_{i}\right.$ is observed $\}$ ), whereas that without responses is called the unlabeled set (i.e., $U=\{1, \cdots, n\}-L$ ). It is often tacitly assumed that the data were subsequently sorted so that the first $|L|$ observations are labeled. If $\boldsymbol{Y}_{L} \in \mathbb{R}^{|L|}$ is the (known) labeled response vector and $\boldsymbol{Y}_{U} \in \mathbb{R}^{|U|}$ is the (unknown) unlabeled response vector, then the data have the partition

$$
\boldsymbol{Y}\left(\boldsymbol{Y}_{U}\right)=\left(\begin{array}{c}
\boldsymbol{Y}_{L} \\
\boldsymbol{Y}_{U}
\end{array}\right), \boldsymbol{X}=\left(\begin{array}{l}
\boldsymbol{X}_{L} \\
\boldsymbol{X}_{U}
\end{array}\right) .
$$

There has been a whole spectrum of interesting ideas on how to learn from both labeled and unlabeled data (Chapelle et al., 2006c). The heuristic method of self-learning (also known as self-training, self-labeling or decision-directed learning) is probably the earliest idea of incorporating unlabeled data during training. The idea is to repeatedly train on the full data using a wrapper algorithm with the unlabeled responses set to their predicted values from the prior iteration, i.e., set $\widehat{\boldsymbol{f}}_{U}^{(0)}=\overrightarrow{0}$ and repeat $\widehat{\boldsymbol{f}}^{(i+1)}=\phi\left(\boldsymbol{Y}\left(\widehat{\boldsymbol{f}}_{U}^{(i)}\right), \boldsymbol{X}\right)$ where $\phi(\boldsymbol{y}, \boldsymbol{X})$ is an arbitrary supervised learner trained from data $(\boldsymbol{y}, \boldsymbol{X})$. Earlier heuristic versions of this approach were introduced in late 1960s (Scudder, 1965; Agarwala, 1970). Although this method seems heuristic, recent work has related these approaches to fixed point optimization and Lipchitz continuity. This form of continuity can provide rigorous explanations for why self-training is expected to work (Culp and Michailidis, 2008, Culp, 2011a).

Some other prior work in semi-supervised learning was cast as a transductive inference problem (Vapnik and Chervonenkis, 1974). This idea is based on an intuitive belief that predicting the unlabeled observations at hand (i.e., available during training) should be 
easier than predicting arbitrary data, which motivates some semi-supervised approaches. Other earlier attempts include semi-supervised extensions of Fisher's linear discriminate rule (Hosmer Jr, 1973; McLachlan and Ganesalingam, 1982), EM algorithm based (Dempster et al., 1977), multinomial mixture models (Cooper and Freeman, 1970), and Gaussian mixture model based probability approximately correct (PAC) learning ideas (Ratsaby and Venkatesh, 1995). With the rising popularity of support vector machines (SVMs), transductive SVMs emerged as an extension to standard SVMs for semi-supervised learning (Joachims, 1999). Transductive SVMs and semi-supervised SVMs (S3VMS) find an imputed labeling for all the unlabeled data, and a separating hyperplane, such that maximum margin, is achieved on the labeled data and (imputed) unlabeled data (Chapelle et al., 2006b; Ji et al., 2008; Li et al., 2010). Graph-based approaches, which are of the focus of this dissertation, are another common class of semi-supervised learning methods (Chapelle et al. 2006c) and are introduced next by first describing the issue of graph construction.

\subsubsection{Proximity Graph Construction}

Semi-supervised graph-based methods have attracted significant interest in recent years (Liu et al., 2010; Chapelle et al., 2006c; Culp and Ryan, 2013). Most notably, they can capture non-linear structures within the feature space often referred to as manifolds and use manifolds if they help predict the response. Proximity graphs can be observed directly, e.g., the data are represented via a connection or a network between close neighboring points such as a social network, a webpage hit set, a citation network, or a protein interaction network. In other cases, graphs can be constructed from a feature data matrix $\boldsymbol{X}$. In either case, existing graph-based semi-supervised methods are designed to bring out the heterogeneous nature of a network.

Proximity graphs can be constructed from a feature data matrix $\boldsymbol{X}$ as follows. First, a distance metric such as Euclidean distance is selected, and a $k$-NN metric is then often 
applied to induce sparsity by setting all the distances beyond the $k$ nearest neighbors to $\infty$. Dissimilarities are then converted to a similarity graph usually by way of some local kernel function. Let $\boldsymbol{G}=(\boldsymbol{V}, \mathbb{E})$ be the resulting proximity graph, where the nodes $\boldsymbol{V}=$ $\{1, \ldots, n\}$ represent the observations and the similarity between pairs of nodes are directly given by a collection of weighted edges $\mathbb{E}$. Methods to come use the corresponding $n \times n$ adjacency matrix $W=\left[w_{i j}\right]$ or graph for short, where $w_{i j}$ is the weight on the edge between observations $i$ and $j$ in graph $\boldsymbol{G}$. The Gram matrix in the $k$-NN method using kernel (2.7) is an example of a proximity graph used by supervised techniques. Semi-supervised learning has an advantage here since the unlabeled data can provide important gap-filling structural connectivity. Whether a graph is observed directly or constructed from a model matrix $\boldsymbol{X}$, it emits the partitioning

$$
\boldsymbol{W}=\left(\begin{array}{ll}
\boldsymbol{W}_{L L} & \boldsymbol{W}_{L U} \\
W_{U L} & \boldsymbol{W}_{U U}
\end{array}\right)
$$

where $\boldsymbol{W}_{L L}$ directly contains labeled-to-labeled adjacencies, $\boldsymbol{W}_{L U}=\boldsymbol{W}_{U L}^{T}$ labeled-to-unlabeled adjacencies, and $\boldsymbol{W}_{U U}$ unlabeled-to-unlabeled adjacencies.

A natural question is "Why should we use a proximity graph?" As motivation, consider the simulated Swiss roll data from Culp (2011b) and its feature data plotted here in Figure 2.1(a). The data having the appearance of a Swiss roll manifold are embedded in a 3D space. In order for semi-supervised learning to work in this setting, we must relate the conditional density of $y_{0} \mid x_{0}$ to the marginal density of $x_{0}$. Take for example the binary gray and black responses in Figure 2.1(a); these were generated deterministically along chunks of the Swiss roll.

It is evident from Figure 2.1(a) that the observed feature data has a non-Euclidean structure. For example, some observations in an inner layer of the roll have a smaller Euclidean distance to observations in an outer layer than to observations in the same chunk of constant 

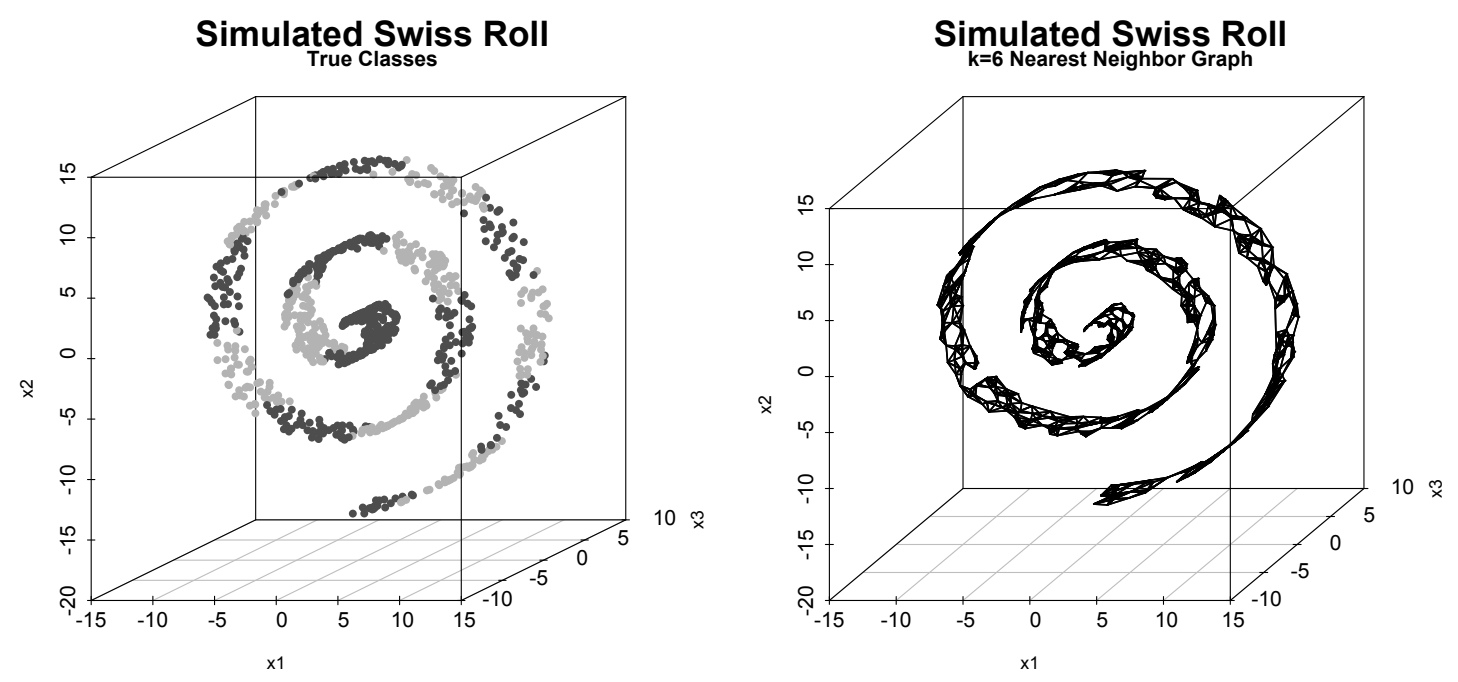

Figure 2.1: (a) Swiss Roll Data Set in Feature Space with Response Regions in a 3D Lattice. (b) Sparse Proximity Graph Approximation of the Swiss Roll.

responses along the roll. In order to capture this structure, we need to effectively walk along the spiral path, and proximity graphs are useful for this endpoint. A $k$-NN graph method with $k=6$ was applied in Figure 2.1(b). Adjacency matrix $\boldsymbol{W}$ was computed from this 6-NN graph with a local kernel.

We make the following observation from the Swiss roll example. If manifolds exist within the feature data, then the graph-based approach does indeed make sense. The Swiss roll is a situation where knowing the manifold can aid in classification, so constructing a proximity graph from $\boldsymbol{X}$ is useful for such problems. However, an even more glaring example can occur when the feature data cluster into unconnected manifolds such that the majority category (in classification) or the expected response (in regression) differs dramatically from manifold to manifold. Take for example the unlabeled maze in Figure 2.2. The response differs on the two sides of the maze. In this type of case in mind, some have invoked the so-called cluster assumption, i.e., two points lie on the same manifold if a network-based distance between them is very low, but such distances between points on separate manifolds 


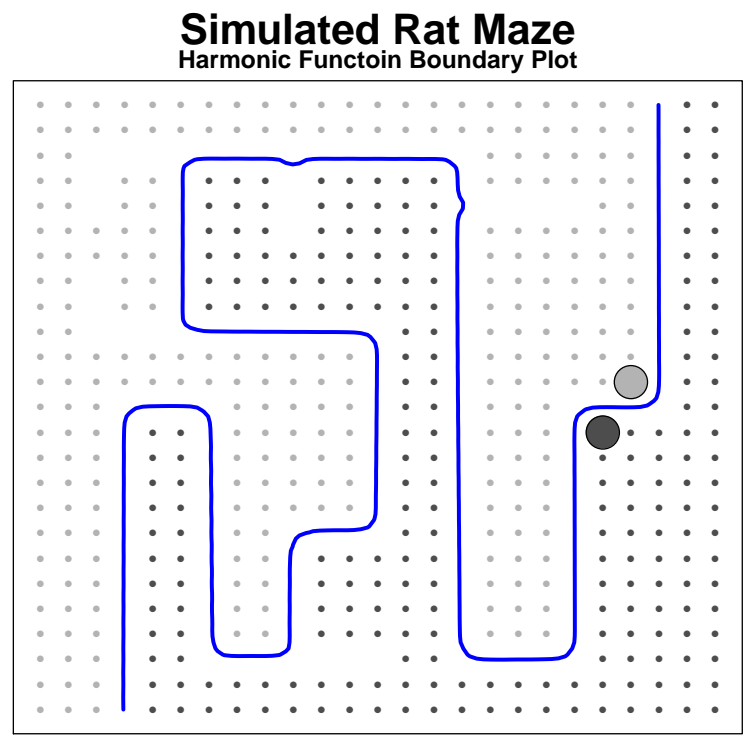

Figure 2.2: A 2D Rat Maze Data Set with $n=487,|L|=2$, and Binary Gray or Black Responses Satisfying the Cluster Assumption.

are usually large. The two sides of the maze are the manifolds, and in terms of language from graph theory, they also correspond to the two connected components in the graph $\boldsymbol{W}$ constructed from this $487 \times 2$ data matrix $\boldsymbol{X}$. In general, a graph-based semi-supervised method relies on the unlabeled data being useful to implicitly discover feature data manifolds that are predictive of the response. These types of graph-based functions certainly do not exhaust all possible semi-supervised functions of $\boldsymbol{X}_{L}, \boldsymbol{Y}_{L}, \boldsymbol{X}_{U}$.

\subsubsection{Semi-Supervised Optimization}

Semi-supervised optimization typically involves generalizing (2.6) to account for the unlabeled data during training. The techniques of how to generalize this optimization problem 
vary. One such example, of primary use in this work, is labeled loss

$$
\min _{\boldsymbol{f}} \mathscr{L}\left(\boldsymbol{Y}_{L}, \boldsymbol{f}_{L}\right)+\lambda \mathbb{J}(\boldsymbol{f}),
$$

which generalizes (2.6) by incorporating unlabeled data through the penalty function (Culp and Ryan, 2013). Another example is joint training

$$
\min _{\boldsymbol{f}, \boldsymbol{Y}_{U}} \mathscr{L}\left(\boldsymbol{Y}\left(\boldsymbol{Y}_{U}\right), \boldsymbol{f}\right)+\boldsymbol{\lambda} \mathbb{J}(\boldsymbol{f})+\boldsymbol{\gamma} \mathbb{P}\left(\boldsymbol{Y}_{U}\right) .
$$

where the (unknown) unlabeled responses are treated as additional decision variables during the optimization, $\mathbb{J}(\boldsymbol{f})$ is a penalty term independent of $\boldsymbol{Y}_{U}, \mathbb{P}\left(\boldsymbol{Y}_{U}\right)$ is penalty term independent on $\boldsymbol{f}$, and $\lambda>0, \gamma>0$ are smoothing parameters.

For labeled loss 2.15$), \mathbb{J}(\boldsymbol{f})$ could be chosen as any penalty function. The most common way to incorporate optimization (2.15) to account for the unlabeled data during training is through general penalty matrices, $\mathbb{J}(\boldsymbol{f})=f^{T} \boldsymbol{B} \boldsymbol{f}$ for some $n \times n$ symmetric positive semidefinite matrix $\boldsymbol{B}$, which is pursued in this work. Similar to the graph $\boldsymbol{W}$, the matrix $\boldsymbol{B}$ emits similar partitions to 2.14,

$$
\boldsymbol{B}=\left(\begin{array}{ll}
\boldsymbol{B}_{L L} & \boldsymbol{B}_{L U} \\
\boldsymbol{B}_{U L} & \boldsymbol{B}_{U U}
\end{array}\right)
$$

and its submatrix $\boldsymbol{B}_{U L}=\boldsymbol{B}_{L U}^{T}$ must be nonzero in order for the unlabeled data to have an effect. Theorem 1 is a general result regarding labeled loss and an arbitrary positive semidefinite matrix $\boldsymbol{B}$.

Theorem 1. Let $\boldsymbol{B}$ be an arbitrary positive semi-definite $n \times n$ penalty matrix such that $\boldsymbol{B}_{U U}$ 
is positive definite. The solution to

$$
\min _{f} \mathscr{L}\left(\boldsymbol{Y}_{L}, \boldsymbol{f}_{L}\right)+\lambda \boldsymbol{f}^{T} \boldsymbol{B} \boldsymbol{f}
$$

is given by

$$
\widehat{f}_{U}=-\boldsymbol{B}_{U U}^{-1} \boldsymbol{B}_{U L} \widehat{\boldsymbol{f}}_{L}
$$

where $\widehat{\boldsymbol{f}}_{L}$ solves

$$
\min _{f_{L}} \mathscr{L}\left(\boldsymbol{Y}_{L}, \boldsymbol{f}_{L}\right)+\lambda \boldsymbol{f}_{L} \boldsymbol{B}_{L L}^{\star} \boldsymbol{f}_{L}
$$

with $\boldsymbol{B}_{L L}^{\star}=\boldsymbol{B}_{L L}-\boldsymbol{B}_{L U} \boldsymbol{B}_{U U}^{-1} \boldsymbol{B}_{U L}$.

Proof. The labeled loss optimization (2.17) can be written as

$$
\min _{\boldsymbol{f}} \mathscr{L}\left(\boldsymbol{Y}_{L}, \boldsymbol{f}_{L}\right)+\lambda\left(\boldsymbol{f}_{L}^{T} \boldsymbol{B}_{L L} \boldsymbol{f}_{L}+2 \boldsymbol{f}_{U}^{T} \boldsymbol{B}_{U L} \boldsymbol{f}_{L}+\boldsymbol{f}_{U}^{T} \boldsymbol{B}_{U U} \boldsymbol{f}_{U}\right)
$$

Differentiating with respect to $f_{U}$ and for any $\boldsymbol{f}_{L}$ yields,

$$
\begin{aligned}
& \Longrightarrow \boldsymbol{B}_{U L} \widehat{\boldsymbol{f}}_{L}+\boldsymbol{B}_{U U} \widehat{\boldsymbol{f}}_{U}=0 \\
& \Longrightarrow \widehat{\boldsymbol{f}}_{U}=-\boldsymbol{B}_{U U}^{-1} \boldsymbol{B}_{U L} \widehat{\boldsymbol{f}}_{L} .
\end{aligned}
$$

For this specific $\widehat{\boldsymbol{f}}_{U}$, we have that

$$
\begin{aligned}
\boldsymbol{f}^{T} \boldsymbol{B} \boldsymbol{f} & =\boldsymbol{f}_{L}^{T} \boldsymbol{B}_{L L} \boldsymbol{f}_{L}+2 \boldsymbol{f}_{U}^{T} \boldsymbol{B}_{U L} \boldsymbol{f}_{L}+\boldsymbol{f}_{U}^{T} \boldsymbol{B}_{U U} \boldsymbol{f}_{U} \\
& =\boldsymbol{f}_{L}^{T} \boldsymbol{B}_{L L} \boldsymbol{f}_{L}-2 \boldsymbol{f}_{L}^{T} \boldsymbol{B}_{L U} \boldsymbol{B}_{U U}^{-1} \boldsymbol{B}_{U L} \boldsymbol{f}_{L}+\boldsymbol{f}_{L}^{T} \boldsymbol{B}_{L U} \boldsymbol{B}_{U U}^{-1} \boldsymbol{B}_{U L} \boldsymbol{f}_{L}
\end{aligned}
$$




$$
=\boldsymbol{f}_{L}^{T} \boldsymbol{B}_{L L}^{\star} \boldsymbol{f}_{L} .
$$

Theorem 1 establishes that the loss function only influences the labeled estimates and the unlabeled estimates are a linear combination of those labeled cases irrespective of loss function. Specific choices of $\boldsymbol{B}$ often depend on the graph $\boldsymbol{W}$ in practice. A simplification of Theorem 1 can be found in Culp and Ryan (2013) and a more general result is given in Culp et al. (2015).

In practice, the general idea for choosing $\boldsymbol{B}$ involves constructing penalty matrices designed from the graph $\boldsymbol{W}$, where $\boldsymbol{W}$ as discussed in Section 2.2.1 is usually either observed directly or is constructed from $\boldsymbol{X}$. Section 2.2.1 also gave examples on how to construct feature data graphs which are useful when the feature data contains manifold(s). Proximity graphs provide the connected networks among the nodes (feature data points) that share some possibly non-Euclidean geometrical structure. Two common choices of $\boldsymbol{B}$ include the normalized and unnormalized Laplacian matrices

$$
\boldsymbol{\Delta}= \begin{cases}\boldsymbol{I}-\boldsymbol{D}^{-1 / 2} \boldsymbol{W} \boldsymbol{D}^{-1 / 2} & \text { (Normalized) } \\ \boldsymbol{D}-\boldsymbol{W} & \text { (Unnormalized) }\end{cases}
$$

where $\boldsymbol{D}=\operatorname{diag}(\boldsymbol{W} \overrightarrow{1})$ is the diagonal degree matrix. Either Laplacian matrix is a sensible choice for characterizing the graph $\boldsymbol{W}$ in labeled loss optimization (2.15), and it is unclear which is better. Naturally, if the cluster assumption is satisfied the means on the manifolds will be propagated through the Laplacian in the expected way (Culp and Ryan, 2013). A similar results holds for the normalized Laplacian as well. 
If the penalty $\mathbb{J}(\boldsymbol{f})=\boldsymbol{f}^{T} \boldsymbol{\Delta} \boldsymbol{f}$, then optimization 2.15 becomes

$$
\min _{f} \mathscr{L}\left(\boldsymbol{Y}_{L}, \boldsymbol{f}_{L}\right)+\lambda f^{T} \boldsymbol{\Delta} f
$$

and by Theorem 1 , the solution for $\boldsymbol{f}_{U}$ when $\boldsymbol{B}=\boldsymbol{\Delta}$ is given in 2.18) as $\widehat{\boldsymbol{f}}_{U}=-\boldsymbol{\Delta}_{U U}^{-1} \boldsymbol{\Delta}_{U L} \widehat{\boldsymbol{f}}_{L}$ where $\widehat{\boldsymbol{f}}_{L}$ solves 2.19). The estimator is an example of a harmonic function in graph-based semi-supervised learning (Culp and Ryan, 2013). Its $\widehat{\boldsymbol{f}}_{U}=\boldsymbol{S} \boldsymbol{f}_{L}$, where $\boldsymbol{S}=-\boldsymbol{\Delta}_{U U}^{-1} \boldsymbol{\Delta}_{U L}$ is a stochastic matrix. In other words, the components of $\widehat{\boldsymbol{f}}_{U}$ are probability weighted averages of predicted values $\widehat{\boldsymbol{f}}_{L}$ on the labeled set and have a Markov Chain interpretation Culp and Ryan, 2013).

A Laplacian-based penalty (normalized or unnormalized) will propagate a class majority (in classification) or an average response (in regression) by manifold in a logical manner (Culp and Ryan, 2013). This is advantageous from a performance standpoint if the cluster assumption is satisfied. For example, the harmonic classification border in Figure 2.2 correctly navigates the maze and separates the two manifolds in this simple example where the response is constant on each manifold. Furthermore, this prediction border is independent of the choice of the observation level loss function $L(\cdot, \cdot)$ in the rat maze example as long as $L(\cdot, \cdot)$ satisfies $L\left(c_{1}, c_{1}\right)<L\left(c_{2}, c_{3}\right)$ for any scalars $c_{1}, c_{2}, c_{3}$ such that $c_{2} \neq c_{3}$.

Unlike $\widehat{f}_{U}$, the choice of a loss function is required in order to determine $\widehat{\boldsymbol{f}}_{L}$ in situations that are not as trivial as the rat maze. The squared error loss function is commonly used in a regression context with $y_{i} \in \mathbb{R}$. In this case, $\min _{f_{L}}\left\|\boldsymbol{Y}_{L}-\boldsymbol{f}_{L}\right\|_{2}^{2}+\lambda \boldsymbol{f}_{L}^{\prime} \boldsymbol{\Delta}_{L L}^{*} \boldsymbol{f}_{L}$, where $\boldsymbol{\Delta}_{L L}^{*}=\boldsymbol{\Delta}_{L L}-\boldsymbol{\Delta}_{L U} \boldsymbol{\Delta}_{U U}^{-1} \boldsymbol{\Delta}_{U L}$, and differentiating this functional with respect to $\boldsymbol{f}_{L}$ and equating to $\overrightarrow{0}$ yields

$$
\begin{aligned}
& \Longrightarrow-\left(\boldsymbol{Y}_{L}-\boldsymbol{f}_{L}\right)+\lambda \boldsymbol{\Delta}_{L L}^{*} \boldsymbol{f}_{L}=0 \\
& \Longrightarrow \widehat{\boldsymbol{f}}_{L}=\left(\boldsymbol{I}+\lambda \boldsymbol{\Delta}_{L L}^{*}\right)^{-1} \boldsymbol{Y}_{L} .
\end{aligned}
$$


Therefore the solution for the equation with squared error loss is,

$$
\left[\begin{array}{c}
\widehat{\boldsymbol{f}}_{L} \\
\widehat{\boldsymbol{f}}_{U}
\end{array}\right]=\left[\begin{array}{r}
\left(\boldsymbol{I}+\lambda \boldsymbol{\Delta}_{L L}^{*}\right)^{-1} \\
-\boldsymbol{\Delta}_{U U}^{-1} \boldsymbol{\Delta}_{U L}\left(\boldsymbol{I}+\lambda \boldsymbol{\Delta}_{L L}^{*}\right)^{-1}
\end{array}\right] \times \boldsymbol{Y}_{L}
$$

Another common example is the logistic loss in classification, i.e., responses are restricted to $y_{i} \in\{0,1\}$. In this case, optimization 2.20 reduces to

$$
\min _{f_{L}}\left\{\sum_{i} \log \left(1+\exp \left(-2\left(2 \boldsymbol{y}_{i}-1\right) \boldsymbol{f}_{i L}\right)\right)+\lambda \boldsymbol{f}_{L}^{\prime} \boldsymbol{\Delta}_{L L}^{*} \boldsymbol{f}_{L}\right\} .
$$

Algorithm 1 summarizes a method for iteratively solving penalized logistic loss optimization (2.21) and is a based on the derivation given next. First, differentiating (2.21) with respect to $f_{i}$ for $i \in L$ yields

$$
\sum_{i \in A_{0}} \frac{2 \exp \left(2 \boldsymbol{f}_{i}\right)}{1+\exp \left(2 \boldsymbol{f}_{i}\right)}+\sum_{i \in A_{1}} \frac{-2 \exp \left(-2 \boldsymbol{f}_{i}\right)}{1+\exp \left(-2 \boldsymbol{f}_{i}\right)}+2 \lambda\left(\Delta_{L L}^{*} f_{L}\right)_{i}=0
$$

where sets $\boldsymbol{A}_{0}=\left\{i \in L: y_{i}=0\right\}$ and $\boldsymbol{A}_{1}=\left\{i \in L: y_{i}=1\right\}$. Then the rearranged score equation

$$
\sum_{i \in L}\left\{\left(1-y_{i}\right) \boldsymbol{g}\left(\boldsymbol{f}_{i}\right)-y_{i}\left(1-\boldsymbol{g}\left(\boldsymbol{f}_{i}\right)\right)+\lambda\left(\Delta_{L L}^{*} \boldsymbol{f}_{L}\right)_{i}\right\}=0
$$

with $\boldsymbol{g}\left(\boldsymbol{f}_{i}\right)=\exp \left(2 \boldsymbol{f}_{i}\right) /\left(1+\exp \left(2 \boldsymbol{f}_{i}\right)\right)$ simplifies to

$$
\boldsymbol{s}\left(\boldsymbol{f}_{L}\right)=-\left(\boldsymbol{Y}_{L}-\boldsymbol{g}\left(f_{L}\right)\right)+\lambda \boldsymbol{\Delta}_{L L}^{*} \boldsymbol{f}_{L}=0
$$

so Newton's method can be used to to solve the gradient score 2.22 with $\ell$ th iteration

$$
\boldsymbol{f}_{L}^{(\ell)}=\boldsymbol{f}_{L}^{(\ell-1)}-\left(\left.\nabla \boldsymbol{s}\left(\boldsymbol{f}_{L}\right)\right|_{\boldsymbol{f}_{L}=\boldsymbol{f}_{L}^{(\ell-1)}}\right)^{-1} \boldsymbol{s}\left(\boldsymbol{f}_{L}^{(\ell-1)}\right)
$$


given some initialization $\boldsymbol{f}_{L}^{(0)}$. It follows that $\nabla \boldsymbol{s}\left(\boldsymbol{f}_{L}^{(\ell)}\right)=\boldsymbol{\omega}^{(\ell)}+\lambda \boldsymbol{\Delta}_{L L}^{*}$, where

$$
\boldsymbol{\omega}^{(\ell)}=\operatorname{diag}\left(\boldsymbol{g}\left(\boldsymbol{f}_{L}^{(\ell)}\right)\left(1-\boldsymbol{g}\left(\boldsymbol{f}_{L}^{(\ell)}\right)\right)\right)
$$

For this final part of the derivation, define $\boldsymbol{S}^{(\ell-1)}=\left(\boldsymbol{\omega}^{(\ell-1)}+\lambda \boldsymbol{\Delta}_{L L}^{*}\right)^{-1} \boldsymbol{\omega}^{(\ell-1)}$. Putting this gradient equation together with 2.22$)$ and 2.23 yields

$$
\begin{aligned}
& \boldsymbol{f}_{L}^{(\ell)}=\boldsymbol{f}_{L}^{(\ell-1)}-\boldsymbol{S}^{(\ell-1)}\left(\boldsymbol{\omega}^{(\ell-1)}\right)^{-1}\left(\boldsymbol{Y}_{L}-\boldsymbol{g}\left(\boldsymbol{f}_{L}^{(\ell-1)}\right)-\lambda \boldsymbol{\Delta}_{L L}^{*} \boldsymbol{f}_{L}^{(\ell-1)}\right) \\
& \boldsymbol{f}_{L}^{(\ell)}=\boldsymbol{S}^{(\ell-1)} \boldsymbol{f}_{L}^{(\ell-1)}-\boldsymbol{S}^{(\ell-1)}\left(\boldsymbol{\omega}^{(\ell-1)}\right)^{-1}\left(\boldsymbol{Y}_{L}-\boldsymbol{g}\left(\boldsymbol{f}_{L}^{(\ell-1)}\right)\right) \\
& \boldsymbol{f}_{L}^{(\ell)}=\boldsymbol{S}^{(\ell-1)} \boldsymbol{Z}^{(\ell-1)} \text { with } \boldsymbol{Z}^{(\ell-1)}=\boldsymbol{f}_{L}^{(\ell-1)}-\left(\boldsymbol{\omega}^{(\ell-1)}\right)^{-1}\left(\boldsymbol{Y}_{L}-\boldsymbol{g}\left(\boldsymbol{f}_{L}^{(\ell-1)}\right)\right),
\end{aligned}
$$

and so Algorithm 1 follows as a method for iteratively solving (2.21).

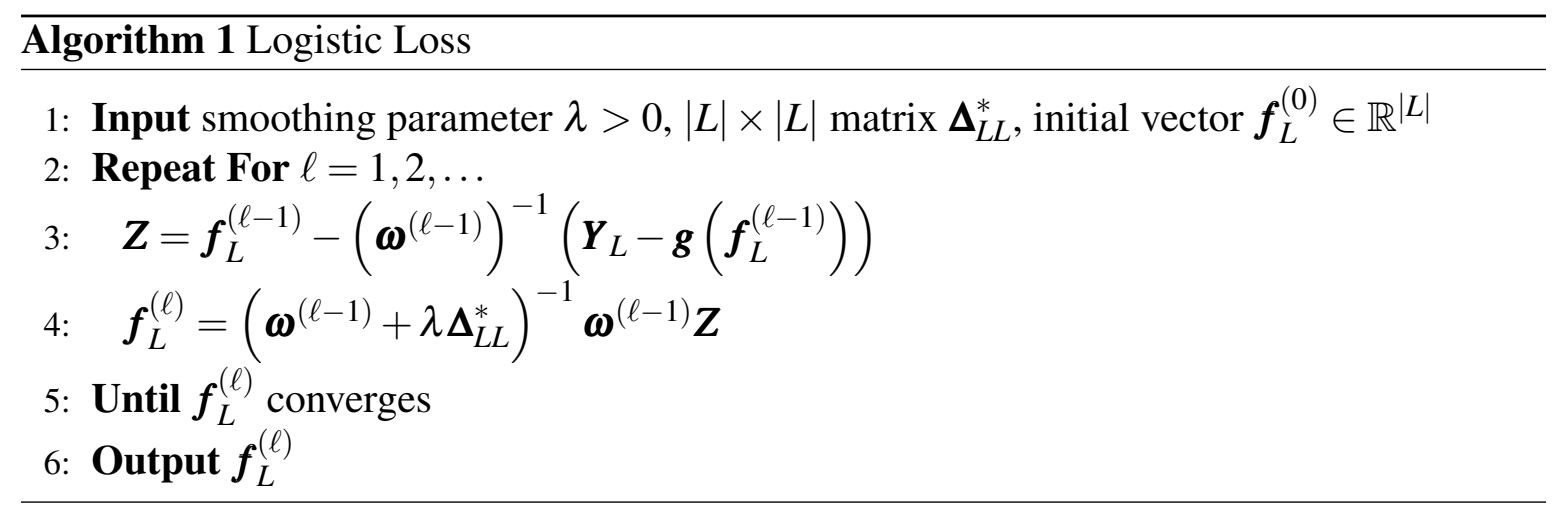

It is observed that the loss function is optimized only on the labeled observations $L$, and we typically expect $|L|$ to be small. So, the choice or complexity of the loss function is not expected to have a significant impact on the computational burden of any resulting semisupervised approaches. Next, Subsection 2.2.3 elaborates on the computational complexities involving graph construction algorithms and will in turn motivate the anchor based graph construction mechanism of Section 2.3 . 


\subsubsection{Computational Issues}

We have thus far reviewed literature on two important aspects of the graph-based semisupervised learning framework: (i) proximity graph construction in Subsection 2.2.1 and (ii) optimization of an objective function in Subsection 2.2.2. A concern about this framework is the issue of scaling with large $n$. As for (i), the time complexity for computing the graph $\boldsymbol{W}$ is $\mathscr{O}\left(n^{2}\right)$ with many graph construction algorithms, and the choice of graph kernel is also important. Commonly used graph kernels include (2.7) and (2.13) which involve the optimization of parameters $k, \lambda, \sigma$. It is noted that $k<7$ is usually adequate, and many implementations default to $k=5$ since $\sigma$ tends to offset the $k$ parameter. As for (ii), there are $\lceil|L| / t\rceil t \approx|L|$ calls to the graph fitting function at each point in a $(\sigma, \lambda)$-grid used during $t$-fold cross-validation, and graph fitting optmization is $\mathscr{O}\left(|L||U|+|U|^{3}\right)$ (Zhu et al., 2003; Zhou et al., 2004b; Belkin et al., 2006). Simplifying the computational burden of (i) graph construction and (ii) graph fitting is important, but care is required in order to not sacrifice the performance.

Graph-based semi-supervised learning approaches often require several matrix operations which becomes tedious with increased $n,|L|$, or $|U|$. The reference BLAS (Basic Linear Algebra Subprograms) typically handles the matrix operations in $\mathrm{R}$ or $\mathrm{C}++$ and speeds up the real computations, but is more tedious to program. Some open source BLAS routines, including OpenBLAS and Apple's optimized BLAS routine vecLib, are also readily available. Consider simulated examples where the components of the $n \times 5$ dimensional feature data $\boldsymbol{X}$ with $n \in\{10,000,60,000\}$ are a random sample from the standard normal distribution, and the labels for the first $|L|=300$ observations are a random sample from the discrete uniform distribution on the set $\{-1,1\}$. In the case of $n=60,000$, the graph construction and optimization were outside of our computational reach. The results in Table 2.1 give some justification as for why this dissertation turns its attention to the graph construction problem in Section 2.3 . 
Table 2.1: Comparison of BLAS Routines in $\mathrm{R}$ when $p=5$ and $|L|=300$.

\begin{tabular}{||l||l||c||c||}
\hline \hline$n$ & R Framework & Graph Construction (in sec.) & Optimization (in sec.) \\
\hline \hline \hline 10,000 & R with reference BLAS & 45.78 & 25.49 \\
10,000 & R + OpenBLAS & 23.08 & 10.08 \\
10,000 & R + vecLib & 21.55 & 08.35 \\
60,000 & R with reference BLAS & NA & NA \\
60,000 & R + OpenBLAS & NA & NA \\
60,000 & R + vecLib & NA & NA \\
\hline \hline
\end{tabular}

\subsection{Anchor Graphs in Semi-Supervised Learning}

Classification and regression problems involving the prediction of the response $y_{0}$ for a new observation $\boldsymbol{x}_{0}$ are challenging yet relevant from a practical standpoint. The graph-based semi-supervised methods of Subsection 2.2.2 used manifolds in the feature data $\boldsymbol{X}$ to predict $y_{0}$ from $x_{0}$ as follows. First, the proximity graphs $\boldsymbol{W}$ of Subsection 2.2.1 incorporated the unlabeled latent network to adequately bring out the heterogeneous nature within the full (labeled and unlabeled) feature data matrix $\boldsymbol{X}$, and this proximity graph $\boldsymbol{W}$ was a fundamental component of the semi-supervised optimization frameworks in Subsection 2.2.2. Subsection 2.2.3 examined the issue of scalability and computational complexity that arises for these graph-based semi-supervised techniques as say $n$ increases. This leads into focus of this Section, i.e., a familiar performance versus speed trade-off: (a) get the best performance by optimizing a computationally intense problem versus (b) get (hopefully) comparable performance results faster by optimizing a problem requiring substantially less computation.

Anchor graphs can be made to strike a good compromise within this trade-off for graphbased semi-supervised learning. This dissertation extends the work of Liu et al. (2010) by presenting a clear description of their anchor graph construction and pairing it with a labeled loss optimization framework that applies to and performs well in large $n$ regression and classification settings. The anchor graph concept uses $m<n$ anchor points spread throughout the feature space to approximate the empirical distribution of the rows of $\boldsymbol{X}$. The fundamental 
matter of selecting the anchor points is the topic of Subsection 2.3.1. Given $m$ anchor points in $\mathbb{R}^{p}$, the Local Anchor Embedding or LAE Algorithm of Subsection 2.3.2 outputs an $n \times m$ matrix $\boldsymbol{Z}$ with non-negative components such that $\boldsymbol{Z} \overrightarrow{1}=\overrightarrow{1}$ that is used to construct an anchor graph.

As long as the selection of the anchors points is handled judiciously, the anchor graph parameter $m$ will be seen to span the performance versus speed trade-off with small (large) $m$ erring toward computational speed (performance). In terms of graph construction for semisupervised learning, the matrix $\boldsymbol{Z}$ will be transformed into a $m \times m$ reduced combinatorial Laplacian matrix in Subsection 2.3.3. This in-turn will also reduce the computational burden of the optimization phase through a reformulated problem to come in Subsection 2.3.4 that optimizes a smaller $m \times 1$ vector $\boldsymbol{\beta}$ instead of the $n \times 1$ vector $\boldsymbol{f}$. The concept of this reformulated problem is the use of the linear approximation $f \approx \boldsymbol{Z} \boldsymbol{\beta}$ in labeled loss optimization (2.15). A complexity analysis in Subsection 2.3.4 will show that small $m$ results in a huge computational saving in both the graph construction and optimization phases of graph-based semi-supervised learning. There is yet another subtle, but substantial computational savings due to anchor graphs during cross-validation. Recall the earlier discussion from Subsection 2.2.1 of constructing the $n \times n$ graph $W$ and the need to pick a kernel function and its bandwidth parameter $\sigma$, e.g., see Gaussian kernel (2.13). It turns out that the anchor graph of Liu et al. (2010) eliminates the $\sigma$ parameter and the need to select a kernel.

The two moons example in Figure 2.3 is used throughout this Section to illustrate the concept of anchor graphs. In this example, there are $|L|=6$ labeled and $|U|=994$ unlabeled cases of feature data in $\mathbb{R}^{p}$ with $p=2$. The labeled cases are all black on the upper moon and gray on the lower moon, so we might prefer the entire upper (lower) moon to be predicted as black (gray) in this simple example of binary classification. It is worth noting that any of the graph-based semi-supervised techniques from Section 2.2 will likely result in this preferred classification out-of-the-box, depending on the implementation of cross-validation 


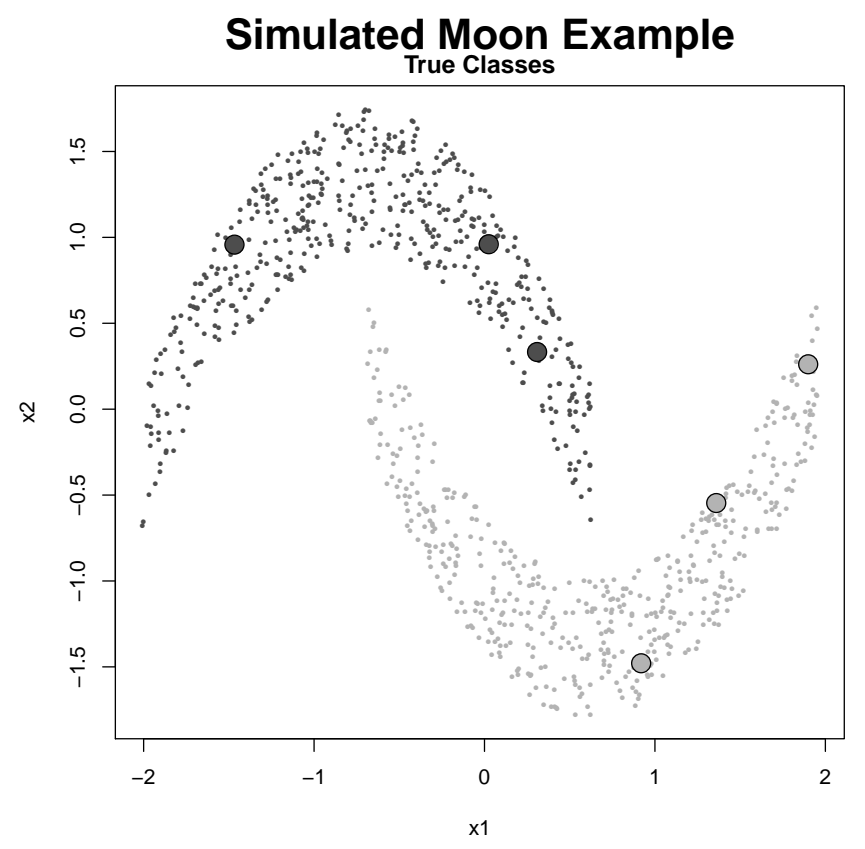

Figure 2.3: A Two Moons Example with $|L|=6$ Labeled Cases and $|U|=994$ Unlabeled Cases. The Moon Determines the True Binary Classification (Black or Gray).

and its ability to accurately estimate tuning parameters. See Culp and Ryan (2013) for a description of using unlabeled data during the necessarily cross-validation phase. The two moons example will also illustrate a key shortcoming of an anchor graph in that it does not necessarily produce this perfect classification without sufficiently large $m$ (and hence additional computational time). In practice, real data sets rarely (if ever) conform to such perfect manifold assumptions like the two moons, but the performance versus computational speed trade-off will be seen to favor the use of anchor graphs on some real data set benchmarks later in Section 2.4

\subsubsection{Anchor Points}

The anchor graph first simplifies graph construction by restricting attention to $m<n$ anchor points or anchors. The idea is to first choose a small set (relative to $n$ ) of $m$ representative 

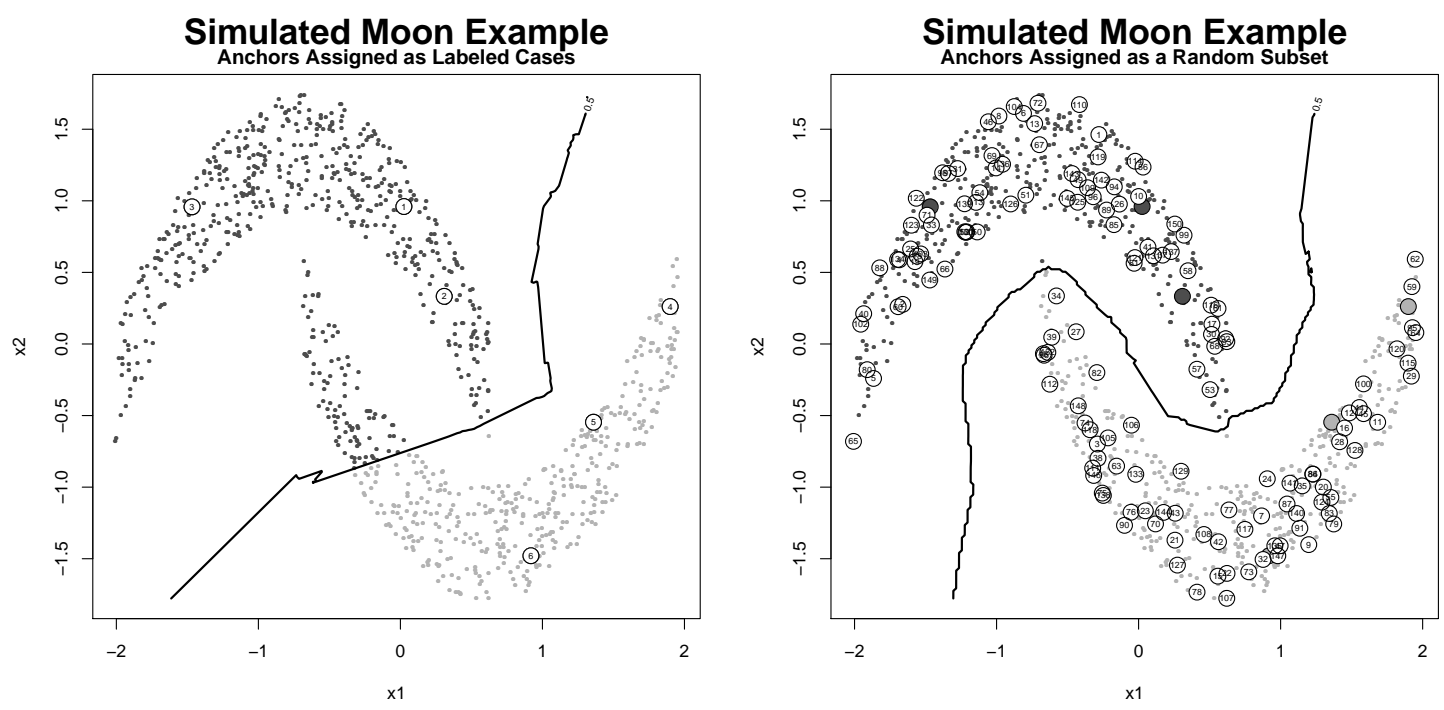

Figure 2.4: Two Moons from Figure 2.3 Revisited. (a) Labeled Cases as Anchors $m=|L|=$ 6. (b) Randomly Selected Rows of $\boldsymbol{X}$ as Anchors $m=150$.

anchors $\boldsymbol{v}_{1}, \ldots, \boldsymbol{v}_{m}$ such that $\boldsymbol{v}_{j} \in \mathbb{R}^{p}$ for all $j=1, \cdots, m$. Let $\boldsymbol{V}$ be the $m \times p$ matrix that stacks the anchors as row vectors. Each anchor represents a neighborhood about a possibly large set of observations in $\boldsymbol{X}$ and are then used for graph construction. Distance calculations are restricted to distances between rows in $\boldsymbol{X}$ and $\boldsymbol{V}$ and results in an $n \times m$ distance matrix. Recall from Subsection 2.2.1 that proximity graph construction involved computation of a distance matrix for all $n$ observations, so this can greatly simplify graph construction. In order to use this, the labeled loss functional (2.15) with the combinatorial Laplacian regularizer must be adapted for processing a non-square distance matrix; this is the topic of Subsection 2.3 .2

The number of anchors and their locations are critical, and a poor choice can lead to inaccurate graphs as empirically demonstrated by revisiting the two moons example in Figure 2.4. In the Figure 2.4(a), the $m=6$ anchors (labeled 1-6 in the figure) are the $|L|=6$ labeled observations. The left horn of the lower moon is misclassified as black because the labeled cases are all away from the horns. This matter is nearly resolved with a much better 

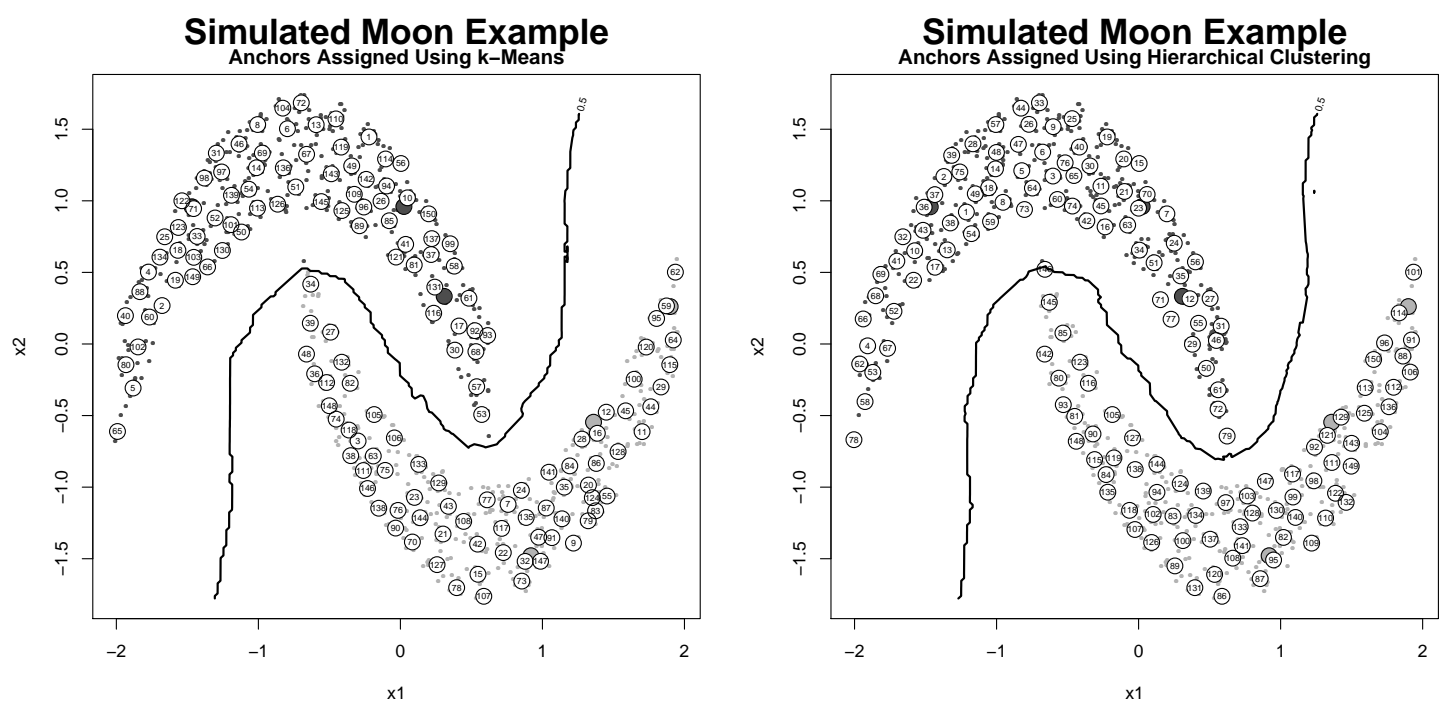

Figure 2.5: Two Moons from Figures 2.3 and 2.4 Revisited. Anchors are the $m=150$ Centroids from (a) $k$-Means and (b) Hierarchical Clustering.

classification in Figure 2.4(b). There are two misclassified cases when $m=150$ anchors are a random sample of the rows from $\boldsymbol{X}$. Notice the jagged nature of both classification borders. This is a direct property of the underlying anchor graph construction and is expected to be more pronounced as $p$ increases.

Clearly, the anchor points should be chosen as a set of points that adequately represent the empirical marginal distribution of the rows of $\boldsymbol{X}$. Typically, the centroids from a cluster algorithm are a good choice for anchor points. Unlike the traditional use of clustering methods where the number of clusters is usually rather small, many clusters may be required in this anchor point application in order to adequately describe any manifolds in the feature data $X$. For example, return to the two moons in Figure 2.5 for demonstrations of anchor point selection by way of $k$-means cluster centers in Figure 2.5 (a) and hierarchical cluster centers in Figure 2.5.(b). Each attempt with $m / n=30 \%$ misclassifies one observation. Notice again the jaggedness of the classification boundaries.

While an adequately large number of anchor points is necessary for representative graph 
construction, a larger number of anchors may be computationally inefficient. In this dissertation, we used $m=\lceil 0.3 n\rceil \times \mathscr{I}_{\{n<1667\}}+500 \times \mathscr{I}_{\{n \geq 1667\}}$ because it was a good compromise of speed versus performance.

\subsubsection{Local Anchor Embedding}

This Subsection concerns the construction of an $n \times m$ matrix $\boldsymbol{Z}$ from the $n \times p$ feature data matrix $\boldsymbol{X}$, such that row $i$ of $\boldsymbol{Z}$ is comprised of the weights for a optimized probability weighted average of the $s$ closest anchor points to feature data $x_{i}$ for $i=1, \ldots, n$. No direct edges/connections exist between the feature data points $\boldsymbol{x}_{1}, \ldots, \boldsymbol{x}_{n}$. Instead, the connectivity among the feature data points are through their common anchors, e.g., if $\boldsymbol{Z}_{i j} \approx \boldsymbol{Z}_{i^{\prime} j} \approx 1$ then $\boldsymbol{x}_{i}$ and $\boldsymbol{x}_{i^{\prime}}$ are both close to anchor point $\boldsymbol{v}_{j}$. The construction of such a $\boldsymbol{Z}$ given by Liu et al. (2010) eliminates the need for graph kernels like (2.7) and (2.13), but introduces the need for a Local Anchor Embedding (LAE) algorithm. Before introducing the actual steps of this algorithm, their LAE algorithm is first introduced conceptually, so its purpose is clear to the reader. Given inputs of an arbitrary $\boldsymbol{x} \in \mathbb{R}^{p}$ and a set of $m$ anchors from Subsection 2.3.1, the purpose of the LAE algorithm is to output a $m$-length vector $z$ like the one outputted from the following simple two-step procedure.

Step 1: Determine a subset of the anchor points $\left\{\boldsymbol{v}_{1}, \ldots, \boldsymbol{v}_{m}\right\}$ of size $s$ that are closest to $\boldsymbol{x} \in \mathbb{R}^{p}$ and denote their indices by $\langle\boldsymbol{x}\rangle \subset \pi_{m}=\{1, \cdots, m\}$. Thus, $|\langle\boldsymbol{x}\rangle|=s$, and if $i \in\langle\boldsymbol{x}\rangle$ and $i^{\prime} \in \pi_{m}$ such that $i^{\prime} \notin\langle\boldsymbol{x}\rangle$, then $\left\|\boldsymbol{x}-\boldsymbol{v}_{i}\right\|_{2}^{2} \leq\left\|\boldsymbol{x}-\boldsymbol{v}_{i^{\prime}}\right\|_{2}^{2}$.

Step 2: Let $\boldsymbol{V}_{\langle\boldsymbol{x}\rangle}$ be the $p \times s$ submatrix of $\boldsymbol{V}^{T}$ consisting of the $s$ anchors closest to $\boldsymbol{x}$ appended as column vectors and define the simplex $\mathbb{S}=\left\{\boldsymbol{z} \in \mathbb{R}^{s}: \overrightarrow{1}^{T} \boldsymbol{z}=1, \boldsymbol{z}_{i} \geq 0\right\}$. The optimization

$$
\min _{z_{\langle x\rangle} \in \mathbb{S}} g\left(z_{\langle x\rangle}\right) \text {, where } g\left(z_{\langle\boldsymbol{x}\rangle}\right)=g\left(\boldsymbol{x}, \boldsymbol{z}_{\langle\boldsymbol{x}\rangle}\right)=\frac{1}{2}\left\|\boldsymbol{x}-\boldsymbol{V}_{\langle\boldsymbol{x}\rangle} z_{\langle\boldsymbol{x}\rangle}\right\|_{2}^{2}
$$


solves for an $\boldsymbol{s}$-length vector $\boldsymbol{z}_{\langle\boldsymbol{x}\rangle}$. The outputted $m$-length vector $\boldsymbol{z}$ takes its entry $\boldsymbol{z}_{i}$ from the corresponding entry in $z_{\langle x\rangle}$ if $i \in\langle x\rangle$ and $z_{i}=0$ otherwise for $i=1, \ldots, m$.

If Steps 1 and 2 above are applied sequentially to the rows of $\boldsymbol{X}$ and the outputted $\boldsymbol{z}$ 's are stacked as row vectors into a matrix, then the matrix $\boldsymbol{Z}$ mentioned earlier results. The LAE algorithm, which uses an iterative numerical approach to carry out Step 2 given above, is given below in Algorithm 4 . The inner workings of Algorithm 4 are complex, but a conceptual understanding of Algorithm 4 is intuitive from geometric and optimization perspectives. Both perspectives are given next to provide deeper insight.

\subsubsection{Geometric Example of the LAE Algorithm}

The geometric perspective of the LAE algorithm involves the convex polytope of all possible probability weighted averages of the $s$ closest anchors to an arbitrary data vector $\boldsymbol{x}$. This convex polytope may include the data vector $x$ in which case $x=\sum_{i=1}^{m} z_{i} v_{i}=\sum_{i \in\langle x\rangle} z_{i} v_{i}$. This happens in the example on right with $p=2$ and $s=4$. The data point $\boldsymbol{x}=(2,3)$ denoted by an empty circle is contained in the convex polygon with edges defined by the anchors labeled 1,2,4 having Cartesian coordinates $(1,2),(2,5),(7,2)$. Anchor 3 with a coordinate of $(3,4)$ does not define an edge of this polygon since it is contained within the convex hull.

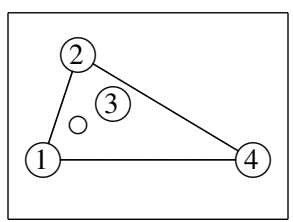
The set $\langle\boldsymbol{x}\rangle=\{1,3,2,4\}$, and matrix

$$
\boldsymbol{V}_{\langle\boldsymbol{x}\rangle}=\left(\begin{array}{llll}
1 & 3 & 2 & 7 \\
2 & 4 & 5 & 2
\end{array}\right)
$$

The vector of probability weights $\boldsymbol{z}_{\langle\boldsymbol{x}\rangle}=(0.53,0.2,0.21,0.06)^{T} \in \mathbb{S}$ was obtained manually 
by brute force and can be used to approximate $x$, i.e.,

$$
\begin{aligned}
\boldsymbol{V}_{\langle\boldsymbol{x}\rangle} \boldsymbol{z}_{\langle\boldsymbol{x}\rangle} & =0.53\left(\begin{array}{l}
1 \\
2
\end{array}\right)+0.21\left(\begin{array}{l}
3 \\
4
\end{array}\right)+0.20\left(\begin{array}{l}
2 \\
5
\end{array}\right)+0.06\left(\begin{array}{l}
7 \\
2
\end{array}\right)=\left(\begin{array}{l}
1.98 \\
3.02
\end{array}\right) \\
& \approx\left(\begin{array}{l}
2 \\
3
\end{array}\right)=x
\end{aligned}
$$

with corresponding criterion error $g\left(\boldsymbol{z}_{\langle\boldsymbol{x}\rangle}\right)=8 \times 10^{-4}$. The LAE algorithm iteratively approximates $\boldsymbol{z}_{\langle\boldsymbol{x}\rangle}=(0.532781,0.1967257,0.2049202,0.06557314)^{T}$ with a better error criterion value of $g\left(\boldsymbol{z}_{\langle\boldsymbol{x}\rangle}\right)=3.25 \times 10^{-10}$.

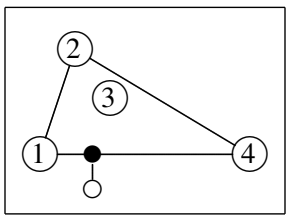

The first example above is a circumstance where the LAE method works in an ideal fashion. Suppose instead that the point $\boldsymbol{x}$ is outside of the convex polytope of its $s$ nearest anchors. Now, $\boldsymbol{x}$ can no longer be recovered exactly as a probability weighted average of its $s$ closest anchors. In this case, the LAE algorithm uses a simplex projection to enforce the constraint that solution $\boldsymbol{z}=\boldsymbol{z}(\boldsymbol{x})$ from optimization 2.24 is in simplex $\mathbb{S}$, and the LAE algorithm effectively finds a vector on the boundary of the convex polygon as close as possible to $x$ in the example on left. The setup of this second example is the same as the first, except $\boldsymbol{x}=(2.5,1)$. The corresponding linear combination that approximates $\boldsymbol{x}$ is

$$
\boldsymbol{V}_{\langle\boldsymbol{x}\rangle} \boldsymbol{z}_{\langle\boldsymbol{x}\rangle}=0.75\left(\begin{array}{l}
1 \\
2
\end{array}\right)+0.00\left(\begin{array}{l}
2 \\
5
\end{array}\right)+0.00\left(\begin{array}{l}
3 \\
4
\end{array}\right)+0.25\left(\begin{array}{l}
7 \\
2
\end{array}\right)=\left(\begin{array}{l}
2.5 \\
2.0
\end{array}\right)
$$

with an error criterion value of $g\left(z_{\langle x\rangle}\right)=1.0$.

Logically, the second example provides a natural solution to the problem of dealing with observations outside of the convex polygon of $s=4$ local anchor points in $\mathbb{R}^{p}$ when $p=$ 2. From a machine learning perspective, this idea still has practical shortcomings. Most 
importantly, vector $\boldsymbol{V}_{\langle\boldsymbol{x}\rangle} \boldsymbol{z}_{\langle\boldsymbol{x}\rangle}$ in the second example is a linear combination of just anchors 1 and 4 even though anchor 4 is the farthest from $\boldsymbol{x}$. In some circumstances, anchor point 4 may not necessarily be on the same manifold as $\boldsymbol{x}$ and anchors $1-3$, so this might put weight on responses from the wrong manifold when constructing predictions. As a result, classification borders (and regression fits) are jagged (e.g., recall discussions of Figures 2.3 . 2.5), and misclassifications may occur on the boundaries of manifolds. The problem is only further compounded by the curse of dimensionality since larger $p$ only increases the odds that points are on the edge and thus fall outside of a convex polytope of $s$ anchors in $\mathbb{R}^{p}$ when $p>2$.

\subsubsection{Optimization Overview of the LAE Algorithm}

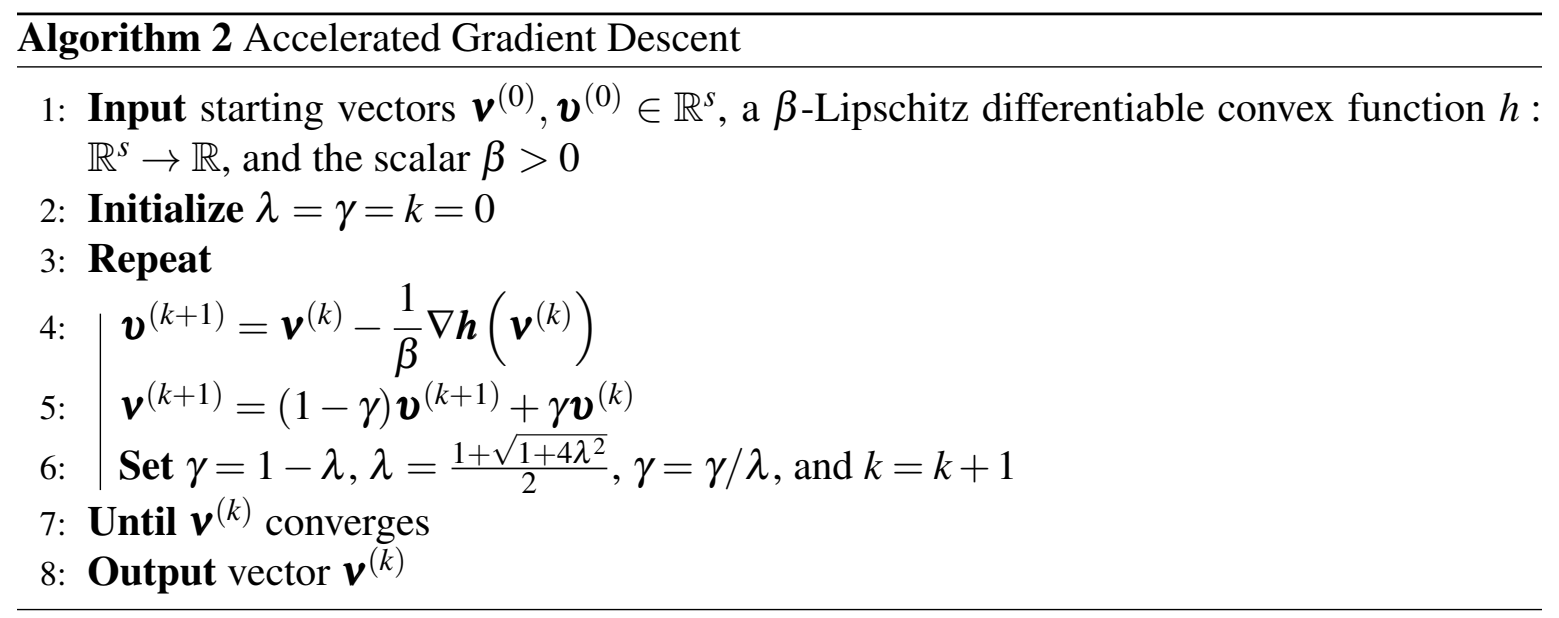

The LAE algorithm is now presented as a hybridized accelerated gradient descent approach that iteratively enforces that its solutions are on simplex $\mathbb{S}$. Direct or approximate calculation of a Hessian matrix is typically required when quadratic programming to solve (2.24), but is computationally expensive. An iterative first-order optimization method based on a simplex projection with an accelerated gradient descent can reduce the computational burden. Interest lies in optimization function $g: \mathbb{R}^{s} \rightarrow \mathbb{R}^{+}$from $(2.24)$ at a given $x \in \mathbb{R}^{p}$, and an accelerated gradient descent method can imply a faster convergence than regular gra- 
dient descent methods (Nesterov, 2005) if this function satisfies the following two sufficient conditions.

1. Function $g(\boldsymbol{z})$ at any fixed $\boldsymbol{x} \in \mathbb{R}^{p}$ is convex and continuously differentiable with respect to $z$.

2. Function $g(\boldsymbol{z})$ at any fixed $\boldsymbol{x} \in \mathbb{R}^{p}$ is $\beta$-Lipschitz, i.e., the gradient

$$
\nabla g\left(z_{\langle x\rangle}\right)=\boldsymbol{V}_{\langle x\rangle}^{T} \boldsymbol{V}_{\langle x\rangle} \boldsymbol{z}_{\langle\boldsymbol{x}\rangle}-\boldsymbol{V}_{\langle\boldsymbol{x}\rangle}^{T} \boldsymbol{x}
$$

satisfies $\left\|\nabla g\left(z_{1}\right)-\nabla g\left(z_{2}\right)\right\|_{1}^{1} \leq \beta\left\|z_{1}-z_{2}\right\|_{1}^{1}$ for any $z_{1}, z_{2} \in \mathbb{R}^{s}$.

Algorithm 2 is the accelerated gradient descent proposed by Yuri Nesterov. Since its output is not constrained to be within simplex $\mathbb{S}$, the projection onto $\mathbb{S}$ outputted by Algorithm 3 , i.e.,

$$
\check{\boldsymbol{z}}=\underset{\boldsymbol{u} \in \mathbb{S}}{\arg \min }\|\boldsymbol{z}-\boldsymbol{u}\|_{2}^{2},
$$

is used in the LAE algorithm of Liu et al. (2010) to force approximate solutions to (2.24) to be within $\mathbb{S}$.

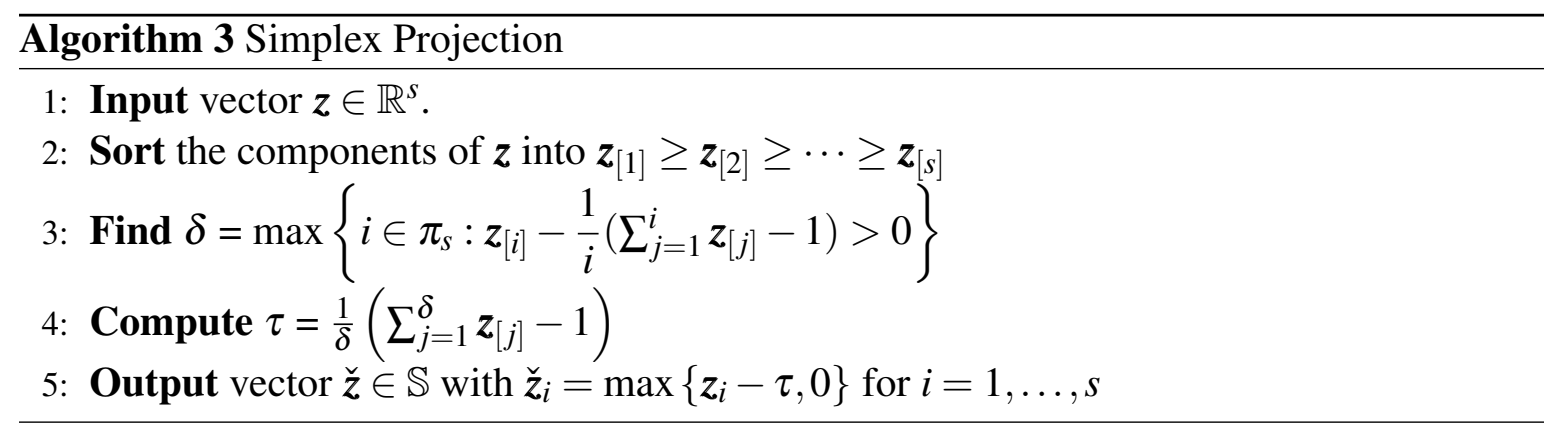

Now, recall the non-negative matrix $\boldsymbol{Z}$ whose $i$ th row consists of the probability weights associating each feature data vector $\boldsymbol{x}_{i}$ for $i=1, \cdots, n$ to its $s$ nearest anchors. When the input is $\boldsymbol{x}_{i}$, the LAE Algorithm 4 outputs the $i$ th row of $\boldsymbol{Z}$ for $i=1, \ldots, n$. Its Step 6 is a simplex projection, and you can see the elements of accelerated gradient descent in Steps 


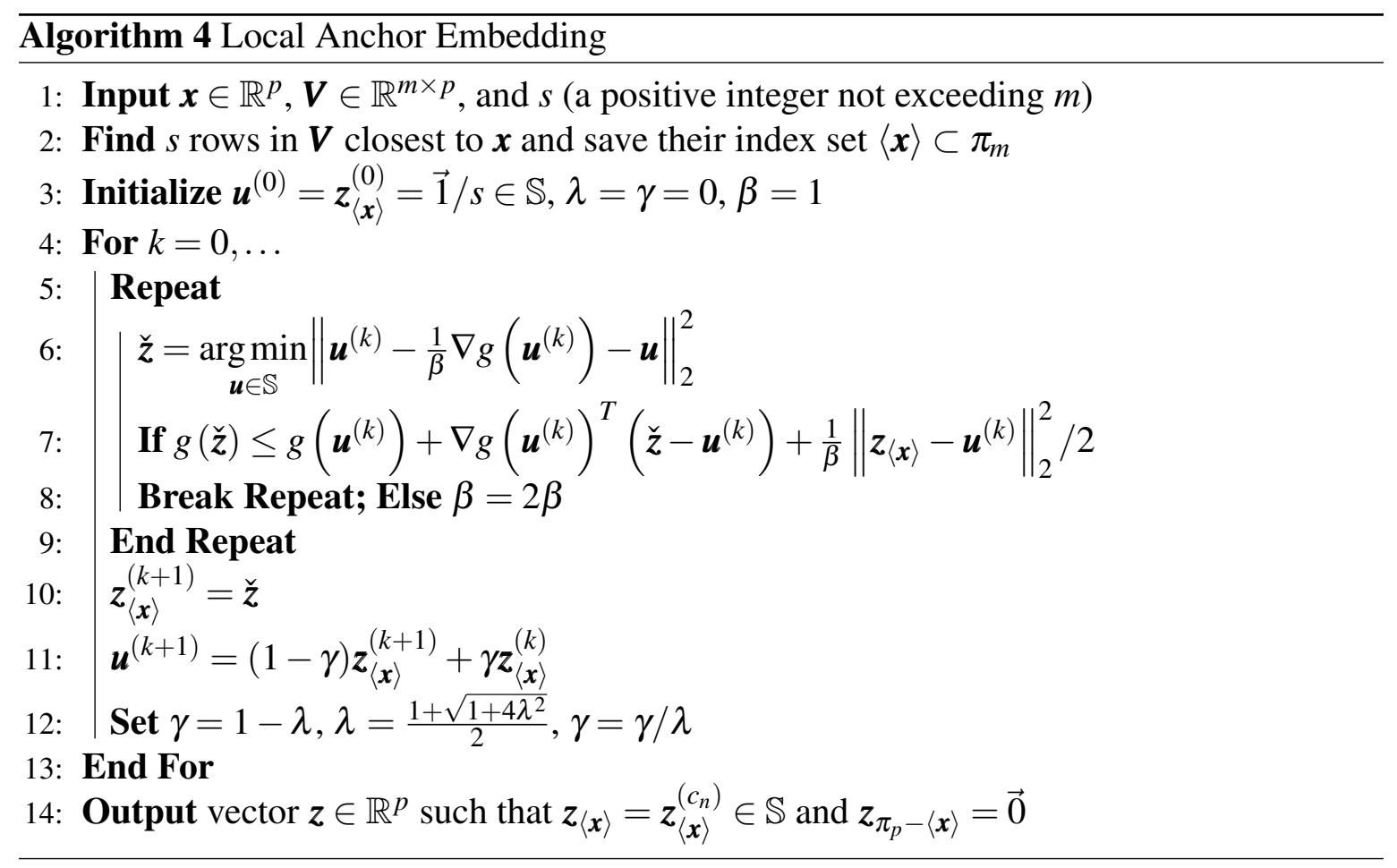

11 and 12. Step 7 looks to reduce the step size $1 / \beta$ until the objective evaluated at the approximate solution within the simplex is no worse than the previous iteration based on a first order linear approximation plus a positive threshold.

\subsubsection{Graph Design}

In the previous Subsection 2.3.2, the LAE Algorithm was documented and deconstructed. This method outputs a non-negative $n \times m$ matrix $\boldsymbol{Z}$ that reduces the dimension of $\boldsymbol{X}$ using $m$ anchor points. A next logical step is to construct an adjacency matrix $\boldsymbol{W}$ and its corresponding graph Laplacian $\Delta$ from $Z$; recall these topics from Subsections 2.2.1 and 2.2.2. The anchor-based graph adjacency matrix is simply

$$
\boldsymbol{W}=Z \Lambda^{-1} Z^{T}
$$



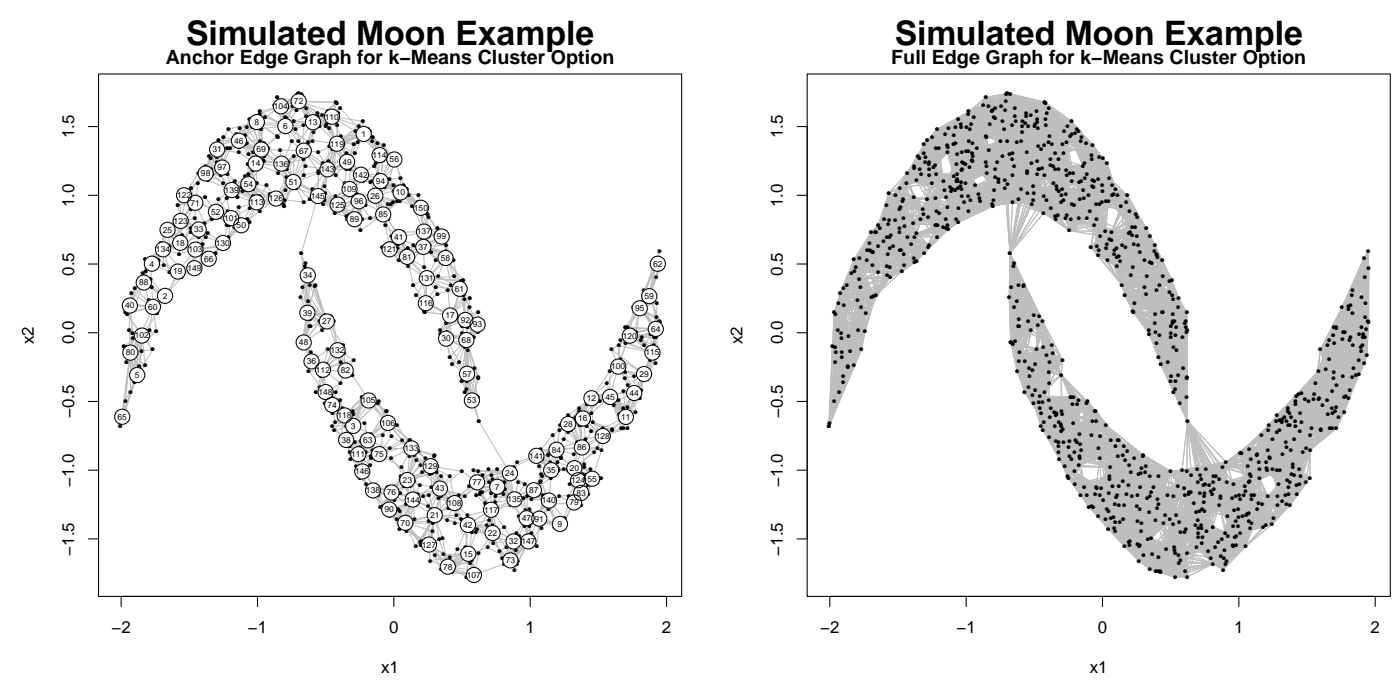

Figure 2.6: Two Moons from Figures 2.3 2.5 Revisited. (a) Anchor Graph Edges Between Anchors $m=150$ and Observations $n=1,000$. (b) Graph $\boldsymbol{W}$ Edges Between Observations.

where $\Lambda=\operatorname{diag}\left(\overrightarrow{1}^{T} \boldsymbol{Z}\right)$ is the diagonal matrix consisting of the column sums of $\boldsymbol{Z}$. This graph forms edges through the anchors. In Figure 2.6 (a), edges are drawn between observation $\boldsymbol{x}_{i}$ and anchor $\boldsymbol{v}_{j}$ if $j \in\left\langle\boldsymbol{x}_{i}\right\rangle$ such that $\boldsymbol{V}_{i j}>0$. In Figure 2.6(b), edges are now drawn between observations $\boldsymbol{x}_{i}$ and $\boldsymbol{x}_{j}$ if $\boldsymbol{W}_{i j}>0$. The panels looks quite similar.

The (unnormalized) graph Laplacian

$$
\boldsymbol{\Delta}=\boldsymbol{D}-\boldsymbol{W}=\boldsymbol{I}-\mathbf{Z} \Lambda^{-1} \boldsymbol{Z}^{T}
$$

follows by construction, since $\boldsymbol{D}=\operatorname{diag}(\boldsymbol{W} \overrightarrow{1})=\boldsymbol{I}$ because $\Lambda^{-1} \boldsymbol{Z}^{T} \overrightarrow{1}=\overrightarrow{1}$ and $\boldsymbol{Z}$ has rows summing to one. This leads to an adjacency matrix well-suited for semi-supervised learning in large $n$ situations because it possesses the following three desirable properties.

1. The $n \times n$ anchor-based graph adjacency matrix $W$ has non-negative components, so its Laplacian $\Delta$ is necessarily positive semi-definite.

2. The sparse weight matrix $\boldsymbol{Z}$ often results in a sparse adjacency matrix $\boldsymbol{W}$ in practice 
because most pairs of data points do not share a common set of nearest anchors.

3. Memory and computation saving result because the $n \times m$ matrix $\boldsymbol{Z}$ is all that is needed to construct the $n \times n$ matrix $\boldsymbol{W}$. However, this can be taken a step further, by computing and storing the smaller $m \times m$ reduced Laplacian matrix $\boldsymbol{Z}^{T} \boldsymbol{\Delta} \boldsymbol{Z}$ directly, i.e., the graph $\boldsymbol{W}$ is never computed in practice.

A heuristic classifier was proposed to obtain classifications using the reduced Laplacian (Liu et al. 2010). Next, this dissertation pursues a more promising optimization framework.

\subsubsection{Regression and Classification with Anchor Graphs}

The labeled loss optimization functional (2.17) is now used to fit the function $f$. Specially, in the special case of $\boldsymbol{f}=\boldsymbol{Z} \boldsymbol{\beta}$ with Laplacian $\Delta=\boldsymbol{I}-\boldsymbol{Z} \Lambda^{-1} \boldsymbol{Z}^{T}$, labeled loss optimization

$$
\min _{f} \mathscr{L}\left(\boldsymbol{Y}_{L}, \boldsymbol{f}_{L}\right)+\lambda \boldsymbol{f}^{T} \boldsymbol{\Delta} f
$$

reduces to

$$
\min _{\boldsymbol{\beta}} \mathscr{L}\left(\boldsymbol{Y}_{L}, \boldsymbol{Z}_{L} \boldsymbol{\beta}\right)+\lambda \boldsymbol{\beta}^{T} \boldsymbol{Z}^{T} \boldsymbol{\Delta} \boldsymbol{\beta}
$$

The solution to 2.25 with squared error loss is generalized ridge regression

$$
\widehat{\boldsymbol{\beta}}=\left(\boldsymbol{Z}_{L}^{T} \boldsymbol{Z}_{L}+\lambda \boldsymbol{Z}^{T} \boldsymbol{\Delta} \boldsymbol{Z}\right)^{-1} \boldsymbol{Z}_{L}^{T} \boldsymbol{Y}_{L}
$$

Logistic loss follows by applying Algorithm 1 with $f=\boldsymbol{Z} \boldsymbol{\beta}$ and $\Delta=\boldsymbol{I}-\boldsymbol{Z} \Lambda^{-1} \boldsymbol{Z}^{T}$. The $\lambda$ parameter controls the semi-supervised smoothness assumption, i.e., $\lambda=0$ the graph is not used while $\lambda>0$ allows the graph to influence the fitted function $\boldsymbol{f}=\boldsymbol{Z} \widehat{\boldsymbol{\beta}}$. A prediction $\boldsymbol{z} \widehat{\boldsymbol{\beta}}$ is computed by applying the LAE algorithm to arbitrary $\boldsymbol{x}$ to get vector $\boldsymbol{z}=\boldsymbol{z}(\boldsymbol{x})$, and this was 
used to determine the classification borders in Figure 2.5 where either category is equally likely.

The anchor graph methodology this Section 2.3 is computationally efficient with a faster convergence rate than other existing graph-based methodologies (Hein et al., 2005, Karlen et al., 2008). One of the main contributions of this method is the use of the $m \times m$ reduced Laplacian matrix $\boldsymbol{Z}^{T} \boldsymbol{\Delta} \boldsymbol{Z}$ in place of the $n \times n$ Laplacian matrix $\boldsymbol{\Delta}$ during optimization. The complexity of the required matrix inverse in the anchor graph method is $\mathscr{O}\left(m^{3}+m^{2} n\right)$ with $m<<n$. This is clearly faster than the computation of 2.20 with squared error loss whose order is $\mathscr{O}\left(|L||U|+|U|^{3}\right)$, so the anchor graph method is promising for large $n$ scenarios from a computational perspective. Table 2.2 rounds out the computational experiment from Subsection 2.2.3. The entries in Table 2.2 are an order of magnitude smaller than the corresponding entries in Table 2.1 and documents the computational efficiency due to anchor graphs.

Table 2.2: Comparison of BLAS Routines in R for the Anchor Graph when $p=5$ and $|L|=300$.

\begin{tabular}{||l||l||c||c||}
\hline \hline \hline$n$ & R Framework & Graph Construction (in sec.) & Optimization (in sec.) \\
\hline \hline \hline 10,000 & R with reference BLAS & 3.41 & 14.71 \\
10,000 & R + OpenBLAS & 1.78 & 0.92 \\
10,000 & R + vecLib & 1.46 & 0.86 \\
60,000 & R with reference BLAS & 22.74 & 88.11 \\
60,000 & R + OpenBLAS & 14.56 & 8.48 \\
60,000 & R + vecLib & 12.74 & 5.91 \\
\hline \hline
\end{tabular}

The classifier is applied to the simulated two moon example using 3-fold cross-validation to estimate $\lambda$ in Figure 2.7 (a). The classification border misclassifies one observation. Furthermore, the fit is jagged which relates directly to LAE algorithm being applied to observations on the edge of a moon (refer to Subsection 2.3.2). The $k$-NN graph with $k=6$ version of labeled logistic loss (Algorithm 1) was also fit with the Laplace kernel $K_{\sigma}\left(\boldsymbol{x}_{i}, \boldsymbol{x}_{j}\right)=\exp \left(-D_{i j} / \sigma\right)$. The parameter combination $(\sigma, \lambda)$ was estimated using 3-fold 

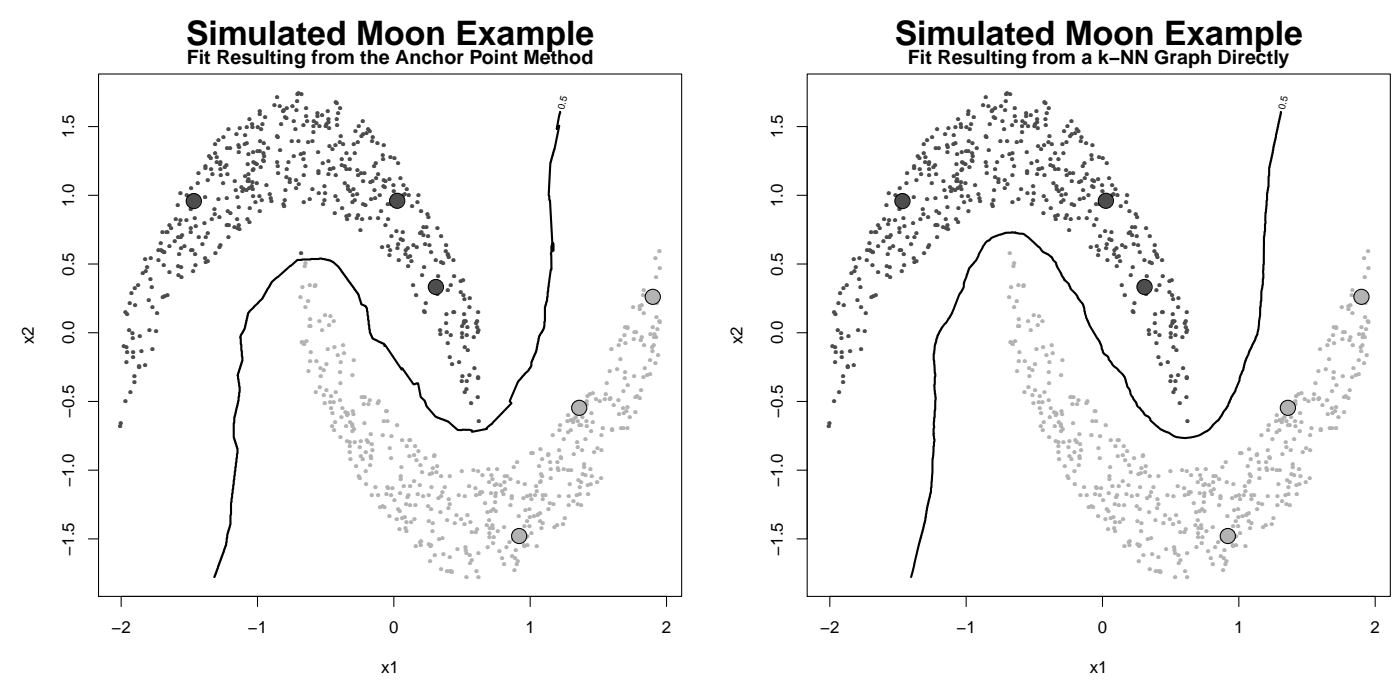

Figure 2.7: Two Moons from Figures 2.3 2.6 Revisited. Prediction Borders Under Logistic Loss with (a) the Anchor Graph Optimization (2.25) and (b) Labeled Loss Optimization (2.15).

cross-validation. The resulting prediction border in Figure 2.7 (b) is smoother with no misclassification errors.

\subsection{Empirical Demonstration}

The competitiveness of three semi-supervised approaches using labeled loss is established on three publicly available datas sets summarized in Table 4.3. Two versions of label loss

Table 2.3: Data Sets.

\begin{tabular}{||l||l||l||l||}
\hline \hline \hline Data Set & $(n, p, m)$ & Type & Reference \\
\hline \hline \hline Meat Spectrometry & $(215,100,64)$ & Regression & $\mid$ Faraway $(2016)$ \\
Power Plant & $(9568,4,500)$ & Regression & Lichman \\
Sonar & $(208,60,62)$ & Classification & Lichman \\
\hline \hline
\end{tabular}

optimization (2.15) were fit with a $k$-NN graph (with $k=6$ ) using cosine distance generated 

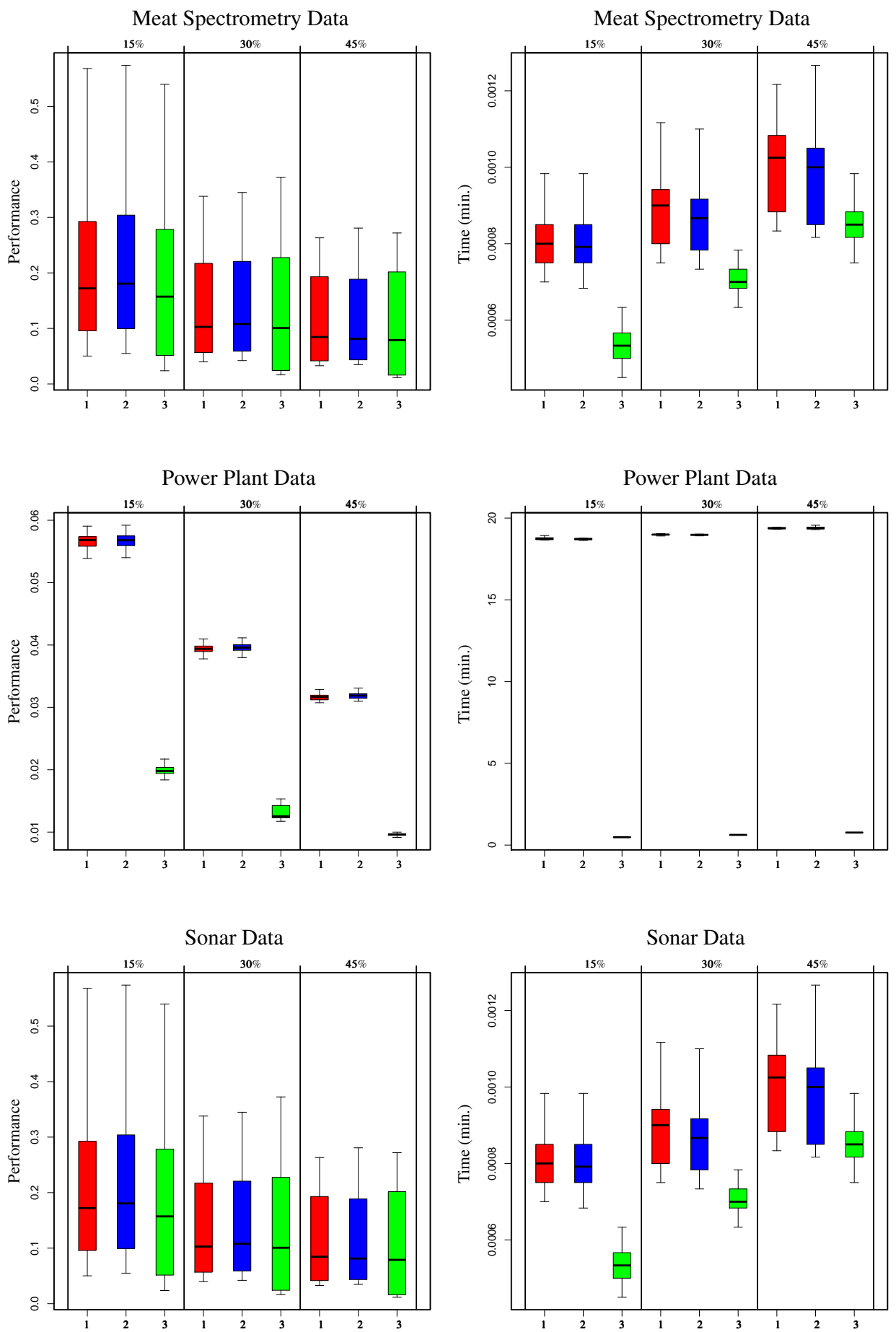

Figure 2.8: Performance (Left) and Time (Right) for Real Data Set Benchmarks. (1) $k$-NN with Laplacian, (2) $k$-NN with Normalized Laplacian, and (3) Anchor Graph. 
from the Laplace kernel function. The two versions only differed in their choice of penalty matrix: (i) Laplacian and (ii) normalized Laplacian. Lagrangian parameter $\lambda$ and kernel parameter $\sigma$ were estimated using 3 -fold cross-validation. The efficient semi-supervised anchor graph approach was fit as the third technique for comparison with $s=5$. The $\lambda$ parameter was estimated via 3-fold cross-validation. In the context of regression, the squared error version was applied. The performance metric was unlabeled root-mean-squared error divided by the standard error of the response. In the context of classification, logistic loss versions were applied with classification error performance metric.

Much work on computational efficiency was done to fit the semi-supervised technique quickly in memory. Since all three approaches were based on inverting a symmetric matrix at some point, a Cholesky decomposition and efficient storage of matrices (e.g., store the upper triangle of the penalty matrix and the lower triangle contains the smoother) in conjunction with optimized LAPACK/ATLAS C-routines boosted speed substantially. An even larger speedup for the $k$-NN graph based approaches was due to the Stagewise Cross Validation (SCV) scheme of Culp et al. (2015).

Performance results and computation times in Figure 2.8 were based on 50 randomly selected labeled sets at each labeled percentage $100|L| / \min \{n, 1500\}=15,30,45 \%$. The anchor method maintains strong performance compared to the other semi-supervised techniques in all cases and is substantially better for the power plan data. A performance boost due to the anchor graph method is not typical or expected in general. The performance difference between the unnormalized and normalized Laplacians is negligible. In terms of time comparisons, the linearity of the anchor graph proved to speed-up the procedure substantially relative to the $k$-NN graph approaches, especially for the larger power plant data set. 


\subsection{Discussion}

Motivation for graph-based semi-supervised techniques typically stems from generalizing a supervised optimization problem. Several supervised minimization problems involve an objective function of the form

\section{"Loss Function” + "Lagrangian Parameter" × "Penalty Function.”}

The choice of loss function depends on the application under examination, but almost always differs based on the response variable type. The main idea is for the loss function to increase as the function deviates from the response. Squared error loss and logistic loss were studied in this research work, but many others could be considered (Hastie et al., 2009). The penalty function accounts for smoothness of the function to be estimated. This is usually constructed in a way so that larger values of the Lagrangian parameter shrink the function towards an extreme endpoint with lower variability and higher bias. For example, ridge regression with a quadratic penalty achieves this trade-off by shrinking the function towards zero, and smoothing splines also achieves this trade-off by shrinking towards a simple linear regression alternative which has lower variance than a non-parametric spline fit. The Lagrangian parameter trades-off predicting the responses well (low bias) versus achieving a stable or smooth estimate (low variance). In practice, estimation of this parameter is critical for the success of a machine learning approach and is almost always optimized via $t$-fold cross-validation.

The graph-based semi-supervised techniques presented in this dissertation were centered around a labeled loss functional as a natural extension to a supervised optimization problem. The goal of this effort was to allow the unlabeled data to influence the fit, and this was accomplished by modifying the smoothness of the fit via the penalty function. This changes the behavior of the fit, especially in cases where manifolds could influence the underlying 
learning process. Penalty functions were constructed to capture these manifolds via a $k$ NN graph operator. Two $k$-NN graph-based approaches were presented. One was based on the combinatorial Laplacian, and the other was based on the normalized Laplacian. Both regularizers have been extensively studied. The role of the Lagrangian tuning parameter still spans the bias/variance trade-off, and the low variance end produces a stable estimator on each manifold, i.e., the function is shrunk towards the mean response in regression (or the majority category in classification) of the manifold. The presented techniques have the advantage of incorporating unlabeled data through a proximity graph, but the disadvantage of the additional computational burden can be dramatic.

An anchor graph approach was presented to approximate these graph-based operators to directly address the computational issues associated with fitting graph operators to big data. This had several advantages. First, an accelerated gradient decent approach summarized in the Local Anchor Embedding or LAE algorithm was shown to dramatically speed up the underlying construction of the graph. Second, a kernel tuning parameter that must be dealt with for $k$-NN graph approaches was removed from the problem and streamlined cross-validation. Third, the approach was demonstrated to perform as good as or better than the $k$-NN graph approaches in some practical applications. The downside of the approach was centered upon the formulation of odd neighborhood structures due to observations that may lie outside of the underlying convex polytopes used to produce the anchor graph. This problem is exacerbated with increased $p$. The main shortcoming of the work is primarily on the heuristic classification technique proposed in Liu et al. (2010). This classifier tends to under-fit the data often performing much worse than standard supervised techniques, semisupervised techniques and frankly, it was the worst technique we compared to in nearly all cases..$^{1}$ Refer to Figure 3.2 in Section 3 and Figure 4.3 in Section 4 . That said the concept of a substantial computational reduction is good, practically, but the authors do not have a

\footnotetext{
${ }^{1}$ An obvious regression extension was used for the continuous responses since the authors do not consider regression.
} 
working technique.

The primary contribution of this dissertation is to build fast semi-supervised techniques based on the anchor graph idea but now the approach will be strongly competitive without sacrificing the fast convergence rate for both graph construction and model optimization. Specifically, in Section 3, a computationally efficient safe version of the general labeled loss optimization and joint training framework is proposed. Representation of a safe multi-view optimization method built on anchor graphs is further proposed in Section 4

Remark 1. Some shortcomings of the original anchor graph technique are of note. First, the K-means cluster centers are primarily used as anchor points, which is known to be unstable with large $n$. Some alternative methodologies for anchor points e.g., cluster centers obtained from any hierarchical clustering method are considered in this dissertation. Figure 2.5 demonstrated the performance of anchor graph method for such a choice and will be further investigated in Appendix A. Second, it is currently unclear how to optimally set the tuning parameters, which is non-trivial, e.g., the number of anchors $m$, closest anchors $s$, and LAE-threshold $c_{n}$. In Appendix A we investigate these issues. Third, the smoothing parameter $\lambda$ was not optimized in prior work. Estimation of the smoothing parameters such as, $\lambda$ in Equation 2.15, $\lambda$ and $\gamma$ in Equation 2.16 is done by cross-validation in this dissertation. Finally, prior work did not adequately address the regression setup. In Chapters 3 and 4 our work build on this prior setup to first improve upon these shortcomings, but once established we are then ready to provide novel improvements in both safe semi-supervised learning (Chapter 3) and multi-view learning (Chapter 4). 


\title{
Chapter 3
}

\section{On Safe Semi-Supervised Learning}

\author{
Mark Vere Culp, Kenneth Joseph Ryan and Prithish BanerJee
}

Submitted in IEEE Transaction on Pattern Analysis and Machine Intelligence

\subsection{Abstract}

Many existing semi-supervised techniques are effective learners only if strong smoothness assumptions hold. These assumptions typically involve a partitioning of the feature space into non-overlapping and possibly non-elliptical clusters. Unlike supervised learners, these semi-supervised techniques essentially predict with the straight arithmetic average or class majority by cluster. While this intuitive cluster assumption is often too strong of a requirement in practice, this does not imply that the unlabeled data have no value for training. It only implies that the unlabeled data used in this fashion degrades performance. Safe semi-supervised learning addresses this issue by adapting "purely semi-supervised" predictions like those described above toward a supervised alternative as needed. Many of the safe methods in the current literature require either accurate density ratio estimation or are 
primarily vetted exclusively on classification problems. As a result, these techniques are impractical on high-dimensional, noisy machine learning problems. The proposed safe semisupervised semi-parametric modeling (S4PM) approach compromises between a supervised and a purely semi-supervised learner, typically boosts performance over these pure alternatives, and is practical for application on real data.

3.1.0.0.1 Keywords: Graph-Based Techniques, Machine Learning, Manifold Assumptions.

\subsection{Introduction}

Consider applications where collecting responses is relatively expensive compared to collecting the corresponding feature information. Data records with complete feature information and the response (labeled data) may be in short supply, while there may be an abundance of observations with missing responses (unlabeled data). Even though supervised techniques are trained from only the labeled data set, these well-developed techniques already: (i) adapt to data with noise in the response or feature space, (ii) select near optimal tuning parameters by CV with robust packages like caret (Kuhn, 2014), and (iii) have computationally efficient implementations. On the other hand, semi-supervised techniques are trained with the labeled and unlabeled data and hold the promise of improved performance through the use of the untapped information within the unlabeled data, but a semi-supervised approach that satisfies (i)-(iii) is necessary in order to compete on noisy real data. This motivates the need for safe semi-supervised approaches that perform comparable to or better than supervised techniques on real data benchmarks (Li and Zhou, 2011; Wang and Chen, 2013; Kawakita and Jun'ichi, 2014).

A learner that finds gaps between regions of feature data is said to satisfy the cluster 
assumption (Chapelle et al., 2006a). Under the cluster assumption, the feature data lie on low dimensional manifolds, and the class or response has low (high) variability within (between) these manifolds (Hein et al., 2005). In other words, these types of so-called semisupervised smoothness assumptions put further restrictions on the true probability model which reinforces the need for these gap finding methods (Lafferty and Wasserman, 2008). Graph cutting (Wang et al., 2013), graph regularization (Zhou et al., 2004a; Belkin et al., 2006; Culp and Ryan, 2013), S3VM methods (Chapelle et al., 2006a, 2008), and several other approaches (Chapelle et al., 2006c) each rely on such assumptions. Henceforth, we refer to these types of gap finding semi-supervised methods as "purely semi-supervised." This paper extends established purely semi-supervised optimization paradigms for graph penalization (Zhou et al., 2004a; Belkin et al., 2006; Chapelle et al., 2006c) into the safe arena. Our approach adapts its smoothness assumption to the characteristics of real data, has real time parameter estimation of nearly all tuning parameters, and is computationally efficient.

Much work has focused on articulating just how bad purely semi-supervised approaches are when semi-supervised assumptions are even slightly perturbed on real data (Culp and Ryan, 2013; Singh et al., 2009). The gap finding mentality of the classifier begins to find non-smooth jagged classifications rules driven primarily by noise and performance deteriorates. This deterioration of performance is profoundly worse than that for supervised learning techniques. This does not bode well for the use of semi-supervised approaches in practice especially given the current level of maturity for supervised learning (Fernández-Delgado et al. 2014). In spite of this, the scale and availability of unlabeled data relative to labeled data makes the use of semi-supervised learning very attractive in applications including drug discovery, text analysis, and bioinformatics.

A novel safe semi-supervised semi-parametric modeling (S4PM) approach that can adapt toward or away from a semi-supervised smoothness assumption is developed in Sections 3.3 and 3.4. Instead of requiring responses to be roughly constant within clusters of feature data 
as discussed above, this is also further relaxed to the residuals between the responses and a supervised fit. When the smoothness assumption fails, an approximate supervised fit results. If the semi-supervised smoothness assumption holds, then the learner is appropriately based on the clusters with a purely semi-supervised fit.

There are many semi-supervised approaches, but few are safe in the manner described here, i.e., they trade-off between a pair of purely supervised and semi-supervised learning approaches. An earlier approach promoted safety by preferring the less-wiggly, supervised fit of non-parametric local kernel regression over a more complicated semi-supervised fit unless overruled by a stepwise criterion (Culp et al., 2009). Recently, existing work typically involved non-noisy structured data problems motivated by either a situation where the ground truth is a large-margin low-density separator (Li and Zhou, 2011) or a kernel density approach is appropriate (Sokolovska et al., 2008, Kawakita and Jun'ichi, 2014; Culp and Ryan, 2013; Azizyan et al., 2013). Furthermore, very few of these approaches are actually implemented, robust, and practical for real data problems. These shortcomings of the other techniques described above help justify why our safe method is advantageous on the practical applications in Section 3.5 .

\subsection{Safe Semi-Supervised Prediction}

Data observations $\left(y_{i}, r_{i}, \boldsymbol{x}_{i}\right) \in \mathbb{R} \times\{0,1\} \times \mathbb{R}^{p}$ for $i=1, \ldots, n$ are a random sample from some joint distribution, where the binary $r_{i}$ indicate whether or not the corresponding response $y_{i} \in \mathbb{R}$ was observed at feature vector $\boldsymbol{x}_{i} \in \mathbb{R}^{p}$. If responses are missing completely at random, then $R_{i} \perp Y_{i} \mid x_{i}$ and the success probability $\pi\left(x_{i}\right)=\pi$ of the Bernoulli trial $R_{i} \mid x_{i}$ does not depend on $\boldsymbol{x}_{i}$. In this case, labeled and unlabeled index sets $L=\left\{i \mid r_{i}=1\right\}$ and $U=\left\{i \mid r_{i}=0\right\}$ are random, but for simplicity, $\left(y_{i}, r_{i}, x_{i}\right)$ were reindexed based on a descending sort by $r_{i}$ so that the first $|L|$ observations are labeled and the remaining $|U|$ are unlabeled. 
In matrix form, the data partition as

$$
\boldsymbol{Y}\left(\boldsymbol{Y}_{U}\right)=\left(\begin{array}{c}
\boldsymbol{Y}_{L} \\
\boldsymbol{Y}_{U}
\end{array}\right) \text { and } \boldsymbol{X}=\left(\begin{array}{c}
\boldsymbol{X}_{L} \\
\boldsymbol{X}_{U}
\end{array}\right)
$$

where $\boldsymbol{Y}_{U}$ is the latent unlabeled responses. For notation, let $\mathscr{N}(\boldsymbol{A})$ be the null space of a matrix $\boldsymbol{A}$ and $\boldsymbol{A} \succeq 0(\boldsymbol{A} \succ 0)$ denote when a symmetric matrix $\boldsymbol{A}$ is positive semi-definite (positive definite).

The focus of this section is the safe semi-supervised approach that solves

$$
\min _{\boldsymbol{f}, \boldsymbol{\beta}}\left\{\sum_{i \in L} c_{i} \mathscr{L}\left(\boldsymbol{y}_{i}, \boldsymbol{x}_{i}^{T} \boldsymbol{\beta}+\boldsymbol{f}_{i}\right)+\lambda_{1} \boldsymbol{f}^{T} \boldsymbol{P}(\boldsymbol{X}) \boldsymbol{f}+\lambda_{2} \boldsymbol{\beta}^{T} \boldsymbol{\beta}\right\},
$$

where the $n \times n$ penalty matrix $\boldsymbol{P}(\boldsymbol{X}) \succeq 0$ accounts for smoothness about observations in $\boldsymbol{X}$, $\mathscr{L}(\cdot, \cdot): \mathbb{R}^{2} \rightarrow \mathbb{R}$ is a loss function, $\boldsymbol{f}$ is a smooth function, $\boldsymbol{\beta}$ is a $p \times 1$ vector of regression coefficients, and $\left\{c_{i}>0\right\}_{i \in L}$ are observation weights. A solution to (3.1) is an example of a safe semi-supervised estimator since it is a compromise between weighted ridge regression $\left(\lambda_{1}=\infty\right)$ and labeled loss graph optimization $\left(\lambda_{2}=\infty\right)$ (Zhou et al., 2004a; Belkin et al. 2006; Culp and Ryan, 2013; Chapelle et al., 2006c).

The matrix $\boldsymbol{P}(\boldsymbol{X})$ with labeled and unlabeled partitioning

$$
\boldsymbol{P}(\boldsymbol{X})=\left(\begin{array}{ll}
\boldsymbol{P}(\boldsymbol{X})_{L L} & \boldsymbol{P}(\boldsymbol{X})_{L U} \\
\boldsymbol{P}(\boldsymbol{X})_{U L} & \boldsymbol{P}(\boldsymbol{X})_{U U}
\end{array}\right)
$$

can be any positive semi-definite penalty matrix although our working examples often form $\boldsymbol{P}(\boldsymbol{X})$ from a proximity graph $\boldsymbol{W}(\boldsymbol{X})$. Two common examples are the graph Laplacian $\boldsymbol{P}(\boldsymbol{X})=\boldsymbol{\Delta}(\boldsymbol{X})$, where $\boldsymbol{\Delta}(\boldsymbol{X})=\boldsymbol{D}(\boldsymbol{X})-\boldsymbol{W}(\boldsymbol{X})$ and $\boldsymbol{D}(\boldsymbol{X})$ is the diagonal row sum matrix of $\boldsymbol{W}(\boldsymbol{X})$, and the normalized Laplacian $\widetilde{\boldsymbol{\Delta}}(\boldsymbol{X})=\boldsymbol{D}(\boldsymbol{X})^{-1 / 2} \boldsymbol{\Delta}(\boldsymbol{X}) \boldsymbol{D}(\boldsymbol{X})^{-1 / 2}$.

A solution $(\widehat{\boldsymbol{f}}, \widehat{\boldsymbol{\beta}})$ to 3.1 can be used to obtain smoothed values $\widehat{\boldsymbol{\eta}}=\boldsymbol{X} \widehat{\boldsymbol{\beta}}+\widehat{\boldsymbol{f}}$ for $\boldsymbol{Y}\left(\boldsymbol{Y}_{U}\right)$. 
Lemma 1 below provides the same smoothed values $\widehat{\boldsymbol{\eta}}$ from an optimization problem in $|U|$ fewer variables. This result is based in-part on the well-known matrix fact: if $\boldsymbol{P}(\boldsymbol{X}) \succeq 0$ and $\boldsymbol{P}(\boldsymbol{X})_{U U}^{-}$is a generalized inverse of $\boldsymbol{P}(\boldsymbol{X})_{U U}$ satisfying $\boldsymbol{P}(\boldsymbol{X})_{U U} \boldsymbol{P}(\boldsymbol{X})_{U U}^{-} \boldsymbol{P}(\boldsymbol{X})_{U U}=$ $\boldsymbol{P}(\boldsymbol{X})_{U U}$, then matrix $\boldsymbol{P}(\boldsymbol{X})_{L U} \boldsymbol{P}(\boldsymbol{X})_{U U}^{-} \boldsymbol{P}(\boldsymbol{X})_{U L}$ is independent of the choice of $\boldsymbol{P}(\boldsymbol{X})_{U U}^{-}$.

Lemma 1. Define the $|L| \times|L|$ matrix

$$
\boldsymbol{P}(\boldsymbol{X})_{L L}^{\star}=\boldsymbol{P}(\boldsymbol{X})_{L L}-\boldsymbol{P}(\boldsymbol{X})_{L U} \boldsymbol{P}(\boldsymbol{X})_{U U}^{-} \boldsymbol{P}(\boldsymbol{X})_{U L}
$$

i.e., the L/U Schur complement of $\boldsymbol{P}(\boldsymbol{X})$. Vectors $\left(\widehat{\boldsymbol{f}}_{L}, \widehat{\boldsymbol{\beta}}\right)$ are a solution to optimization problem

$$
\min _{\boldsymbol{f}_{L}, \boldsymbol{\beta}}\left\{\sum_{i \in L} c_{i} \mathscr{L}\left(\boldsymbol{y}_{i}, \boldsymbol{x}_{i}^{T} \boldsymbol{\beta}+\boldsymbol{f}_{i}\right)+\lambda_{1} \boldsymbol{f}_{L}^{T} \boldsymbol{P}(\boldsymbol{X})_{L L}^{\star} \boldsymbol{f}_{L}+\lambda_{2} \boldsymbol{\beta}^{T} \boldsymbol{\beta}\right\}
$$

if and only if $\left(\widehat{\boldsymbol{f}}_{L}, \widehat{\boldsymbol{\beta}}\right)$ with $\widehat{\boldsymbol{f}}_{U}$ is a solution to 3.1 for any $\widehat{\boldsymbol{f}}_{U}$ satisfying the harmonic property

$$
\boldsymbol{P}(\boldsymbol{X})_{U U} \widehat{\boldsymbol{f}}_{U}=-\boldsymbol{P}(\boldsymbol{X})_{U L} \widehat{\boldsymbol{f}}_{L}
$$

Proof. The objective from 3.1 is proportional to $2 \boldsymbol{f}_{U}^{T} \boldsymbol{P}(\boldsymbol{X})_{U L} \boldsymbol{f}_{L}+\boldsymbol{f}_{U}^{T} \boldsymbol{P}(\boldsymbol{X})_{U U} \boldsymbol{f}_{U}$ as a function of $f_{U}$, and the $f_{U}$ (derivative) score of this expression yields $(3.2)$, which is independent of the loss function, the case weights, and the regression coefficients. Based on applying (3.2) multiple times, vector $f_{U}$ is profiled out of (3.1) through the identity

$$
\begin{aligned}
\boldsymbol{f}^{T} \boldsymbol{P}(\boldsymbol{X}) \boldsymbol{f} & =\boldsymbol{f}_{L}^{T} \boldsymbol{P}(\boldsymbol{X})_{L L} \boldsymbol{f}_{L}+2 \boldsymbol{f}_{U}^{T} \boldsymbol{P}(\boldsymbol{X})_{U L} \boldsymbol{f}_{L}+\boldsymbol{f}_{U}^{T} \boldsymbol{P}(\boldsymbol{X})_{U U} \boldsymbol{f}_{U} \\
& =\boldsymbol{f}_{L}^{T} \boldsymbol{P}(\boldsymbol{X})_{L L} \boldsymbol{f}_{L}-\boldsymbol{f}_{U}^{T} \boldsymbol{P}(\boldsymbol{X})_{U U} \boldsymbol{P}(\boldsymbol{X})_{U U}^{-} \boldsymbol{P}(\boldsymbol{X})_{U U} \boldsymbol{f}_{U} \\
& =\boldsymbol{f}_{L}^{T} \boldsymbol{P}(\boldsymbol{X})_{L L} \boldsymbol{f}_{L}-\boldsymbol{f}_{L}^{T} \boldsymbol{P}(\boldsymbol{X})_{L U} \boldsymbol{P}(\boldsymbol{X})_{U U}^{-} \boldsymbol{P}(\boldsymbol{X})_{U L} \boldsymbol{f}_{L}
\end{aligned}
$$




$$
=\boldsymbol{f}_{L}^{T} \boldsymbol{P}(\boldsymbol{X})_{L L}^{\star} \boldsymbol{f}_{L}
$$

If $\boldsymbol{P}(\boldsymbol{X})_{U U}$ is noninvertible, then each component of $\widehat{\boldsymbol{f}}_{U}$ is not well-defined by 3.2 . By Lemma 1 , the same smoothed values $\widehat{\boldsymbol{\eta}}$ can result from either (3.1) or from 4.3 with (3.2). For example, if 4.3 has the unique solution $\left(\widehat{\boldsymbol{f}}_{L}, \widehat{\boldsymbol{\beta}}\right)$ and $\boldsymbol{P}(\boldsymbol{X})_{U U} \succ 0$, then the smoothed values $\widehat{\boldsymbol{\eta}}$ from either method are

$$
\left(\begin{array}{c}
\widehat{\boldsymbol{\eta}}_{L} \\
\widehat{\boldsymbol{\eta}}_{U}
\end{array}\right)=\left(\begin{array}{c}
\widehat{\boldsymbol{f}}_{L}+\boldsymbol{X}_{L} \widehat{\boldsymbol{\beta}} \\
-\boldsymbol{P}(\boldsymbol{X})_{U U}^{-1} \boldsymbol{P}(\boldsymbol{X})_{U L} \widehat{\boldsymbol{f}}_{L}+\boldsymbol{X}_{U} \widehat{\boldsymbol{\beta}}
\end{array}\right) .
$$

For any loss function, Lemma 1 also establishes that the loss function directly influences just the labeled smoothed values $\widehat{\boldsymbol{\eta}}_{L}$ and then in-turn $\widehat{\boldsymbol{\eta}}_{L}$ determines the unlabeled smoothed values $\widehat{\boldsymbol{\eta}}_{U}$ through the generalized harmonic manipulation 3.2. We view optimization problem (4.3) with smoothness matrix $\boldsymbol{P}(\boldsymbol{X})_{L L}^{\star}$ as a semi-parametric approach. Next, the focus is on regression with the squared error loss $\mathscr{L}\left(y_{i}, \widehat{\boldsymbol{\eta}}_{i}\right)=\left(y_{i}-\widehat{\boldsymbol{\eta}}_{i}\right)^{2}$. Proposition 1 below provides the closed-form matrix solution $\left(\widehat{\boldsymbol{f}}_{L}, \widehat{\boldsymbol{\beta}}\right)$ to the squared error loss version of 4.3 .

Proposition 1. Define the diagonal $|L| \times|L|$ matrix $\boldsymbol{C}_{L L}$ by $\left(\boldsymbol{C}_{L L}\right)_{i i}=c_{i}$ and the $|L| \times|L|$ matrices $\boldsymbol{S}_{L L}=\left(\boldsymbol{C}_{L L}+\lambda_{1} \boldsymbol{P}(\boldsymbol{X})_{L L}^{\star}\right)^{-1} \boldsymbol{C}_{L L}$ and $\tilde{\boldsymbol{S}}_{L L}=\boldsymbol{C}_{L L}\left(\boldsymbol{I}-\boldsymbol{S}_{L L}\right)$. If $\lambda_{2}>0$ or rank $\left(\boldsymbol{X}_{L}\right)=$ $p$, then the unique solution $\left(\widehat{f}_{L}, \widehat{\boldsymbol{\beta}}\right)$ to optimization 4.3 with the squared error loss is

$$
\begin{aligned}
\widehat{\boldsymbol{\beta}} & =\left(\boldsymbol{X}_{L}^{T} \tilde{\boldsymbol{S}}_{L L} \boldsymbol{X}_{L}+\lambda_{2} \boldsymbol{I}\right)^{-1} \boldsymbol{X}_{L}^{T} \tilde{\boldsymbol{S}}_{L L} \boldsymbol{Y}_{L} \\
\widehat{\boldsymbol{f}}_{L} & =\boldsymbol{S}_{L L}\left(\boldsymbol{Y}_{L}-\boldsymbol{X}_{L} \widehat{\boldsymbol{\beta}}\right)
\end{aligned}
$$

Proof. The $\boldsymbol{f}_{L}$-score of 4.3 is $-\boldsymbol{C}_{L L}\left(\boldsymbol{Y}_{L}-\widehat{\boldsymbol{f}}_{L}-\boldsymbol{X}_{L} \widehat{\boldsymbol{\beta}}\right)+\lambda_{1} \boldsymbol{P}(\boldsymbol{X})_{L L}^{\star} \widehat{\boldsymbol{f}}_{L}=\overrightarrow{0}$, so $\widehat{\boldsymbol{f}}_{L}=\boldsymbol{S}_{L L}\left(\boldsymbol{Y}_{L}-\boldsymbol{X}_{L} \widehat{\boldsymbol{\beta}}\right)$. The form for $\widehat{\boldsymbol{\beta}}$ follows from plugging-in this form for $\widehat{\boldsymbol{f}}_{L}$ into the $\boldsymbol{\beta}$-score of 4.3 . 
The smoothed values $\widehat{\boldsymbol{\eta}}=\widehat{\boldsymbol{f}}+\boldsymbol{X} \widehat{\boldsymbol{\beta}}$ from 3.4 are based on an arbitrary loss function. Iterative applications of Proposition 1 (based on weighted squared error loss functions) will converge to this $\widehat{\eta}$ for a broader class of convex loss functions (Culp et al., 2009) including the logistic and Poisson loss functions.

A novelty of this research direction is the simultaneous inclusion of the linear term $\boldsymbol{X}_{L} \boldsymbol{\beta}$ and the non-parametric term $f_{L}$ in the labeled loss functional. It is shown in Section 3.3.1 that the optimal $\widehat{f}$ exploits gaps in the feature data and favors vectors in $\mathscr{N}(\boldsymbol{P}(\boldsymbol{X}))$ close to a weighted mean of the labeled residuals $\boldsymbol{Y}_{L}-\boldsymbol{X}_{L} \boldsymbol{\beta}$ as opposed to $\boldsymbol{Y}_{L}$ by feature data cluster. This added flexibility accounts for the noted improvements in the performance results of Section 3.5 .

\subsubsection{The Cluster Assumption Applied to Residuals}

In Section 4.2, a gap finding property of purely semi-supervised learners was discussed. This subsection rigorously establishes this property in the context of the proposed safe semisupervised approach. For now, let $\boldsymbol{P}(\boldsymbol{X})=\boldsymbol{\Delta}(\boldsymbol{X})$. A cluster assumption learner (Culp and Ryan, 2013) implicitly uses gaps in the full feature data set $\boldsymbol{X}$ to partition the data into subsets $\omega_{i}$ such that $\bigcup_{i} \omega_{i}=L \cup U$ and $\omega_{i} \cap \omega_{j}=\emptyset$ if $i \neq j$. This partitioning follows from favoring vectors $\boldsymbol{f} \in \mathscr{N}(\boldsymbol{\Delta}(\boldsymbol{X}))$ with components $\boldsymbol{f}_{\omega_{i}}$ for subset $\omega_{i}$ consisting of a weighted mean of the available responses $\boldsymbol{y}_{L \cap \omega_{i}}$, but an $\boldsymbol{f}_{U}$ constructed in this fashion may still not be a good predictor of $\boldsymbol{Y}_{U}$ due to trends within the subsets $\omega_{i}$. The proposed semi-parametric

model relaxes this requirement to the residuals $\hat{\boldsymbol{\varepsilon}}_{L}=\boldsymbol{Y}_{L}-\boldsymbol{X}_{L} \widehat{\boldsymbol{\beta}}$ instead of $\boldsymbol{Y}_{L}$ itself and also applies to an arbitrary penalty matrix $\boldsymbol{P}(\boldsymbol{X}) \succeq 0$ as opposed to just the Laplacian as a second generalization of (Culp and Ryan, 2013).

The simple binary graph $\boldsymbol{W}(\boldsymbol{X})$ on the right with $\boldsymbol{P}(\boldsymbol{X})=\boldsymbol{\Delta}(\boldsymbol{X})$ helps illustrate the concepts from the previous paragraph before we present a general theoretical result. This full graph in $n=8$ vertices has null spaces 


$$
\begin{aligned}
& \begin{array}{llllllllllllllll}
1 & 2 & \underline{3} & 4 & \underline{5} & \underline{6} & \underline{7} & \underline{8} & \underline{1} & \underline{2} & \underline{3} & 4 & \underline{5} & \underline{6} & \underline{7} & \underline{8}
\end{array} \\
& \mathscr{N}(\boldsymbol{\Delta}(\boldsymbol{X}))=\operatorname{span}\left\{(1,0,0,1,1,1,0,0)^{T},(0,1,1,0,0,0,1,1)^{T}\right\} \\
& \underline{1} \underline{2} \underline{3} \quad \underline{1} \underline{2} \underline{3} \\
& \mathscr{N}\left(\boldsymbol{\Delta}(\boldsymbol{X})_{L L}^{\star}\right)=\operatorname{span}\left\{(1,0,0)^{T},(0,1,1)^{T}\right\} .
\end{aligned}
$$

A basis for $\mathscr{N}(\boldsymbol{\Delta}(\boldsymbol{X}))$ corresponds to partition $\{\{1,4,5,6\},\{2,3,7,8\}\}$ of all eight vertices based on the connected components of the full graph. Partition $\{\{1\},\{2,3\}\}$ of $L=\{1,2,3\}$ clearly corresponds to the basis for $\mathscr{N}\left(\boldsymbol{\Delta}(\boldsymbol{X})_{L L}^{\star}\right)$

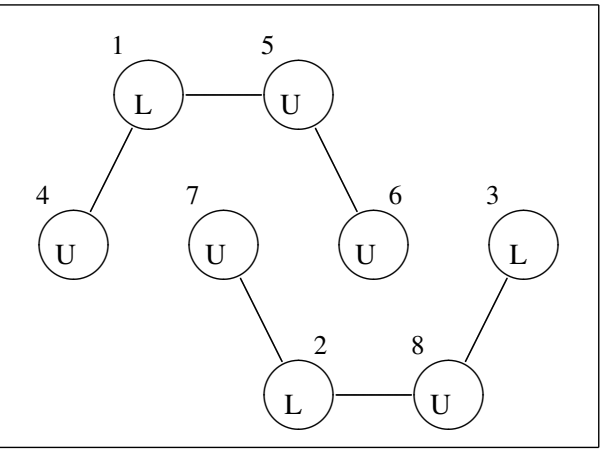
given above, and this partition of connected labeled vertices from the full graph is obtained by simply removing the unlabeled vertices $U=\{4,5,6,7,8\}$ from $\{\{1,4,5,6\},\{2,3,7,8\}\}$. These null spaces characterize the vectors $\boldsymbol{f}$ and $\boldsymbol{f}_{L}$ that do not get penalized in 3.1 and 4.3 . To see this, let $\boldsymbol{v}=(0,1,1,0,0,0,1,1)^{T}$ be the second basis vector for $\mathscr{N}(\boldsymbol{\Delta}(\boldsymbol{X}))$, so $\boldsymbol{v}_{L}=(0,1,1)^{T} \in \mathscr{N}\left(\boldsymbol{\Delta}(\boldsymbol{X})_{L L}^{\star}\right)$. Since $\boldsymbol{\Delta}(\boldsymbol{X}) \boldsymbol{v}=\overrightarrow{0}$ and $\boldsymbol{\Delta}(\boldsymbol{X})_{L L}^{\star} \boldsymbol{v}_{L}=\overrightarrow{0}$, adding any multiple of $\boldsymbol{v}$ to $\boldsymbol{f}$ or of $\boldsymbol{v}_{L}$ to $f_{L}$ incurs no additional penalty in (3.1) or (4.3). By Proposition 2 below, the effect of (3.2) after adding $b \boldsymbol{v}_{L}$ to $f_{L}$ for some scalar $b$ is to add a vector $\tilde{\boldsymbol{v}}_{U}$ to $\boldsymbol{f}_{U}$ such that $\left(b \boldsymbol{v}_{L}^{T}, \tilde{\boldsymbol{v}}_{U}^{T}\right)^{T} \in \mathscr{N}(\boldsymbol{\Delta}(\boldsymbol{X}))$, so $\tilde{\boldsymbol{v}}_{U}=b \boldsymbol{v}_{U}$ in this example. Sets of vectors $\boldsymbol{f}$ or $\boldsymbol{f}_{L}$ that are penalized equally in 3.1 or (4.3) for arbitrary $\boldsymbol{P}(\boldsymbol{X})$ also follows from Proposition 2 .

Proposition 2. If $\boldsymbol{P}(\boldsymbol{X}) \succeq 0$, then $\boldsymbol{f} \in \mathscr{N}(\boldsymbol{P}(\boldsymbol{X})) \Longleftrightarrow \boldsymbol{f}_{L} \in \mathscr{N}\left(\boldsymbol{P}(\boldsymbol{X})_{L L}^{\star}\right)$ and $\boldsymbol{P}(\boldsymbol{X})_{U U} \boldsymbol{f}_{U}=$ $-\boldsymbol{P}(\boldsymbol{X})_{U L} \boldsymbol{f}_{L}$

Proof. The representation $\boldsymbol{A}=\boldsymbol{B}^{T} \boldsymbol{B}$ exists for any (symmetric) matrix $\boldsymbol{A} \succeq 0$ by its eigendecomposition and non-negative eigenvalues, so $\boldsymbol{v} \in \mathscr{N}(\boldsymbol{A})$ if and only if $\boldsymbol{v}^{T} \boldsymbol{A} \boldsymbol{v}=\boldsymbol{v}^{T} \boldsymbol{B}^{T} \boldsymbol{B} \boldsymbol{v}=$ 0. Now, if $\boldsymbol{P}(\boldsymbol{X})_{U U} \boldsymbol{f}_{U}=-\boldsymbol{P}(\boldsymbol{X})_{U L} \boldsymbol{f}_{L}$, then $\boldsymbol{f}^{T} \boldsymbol{P}(\boldsymbol{X}) \boldsymbol{f}=\boldsymbol{f}_{L}^{T} \boldsymbol{P}(\boldsymbol{X})_{L L}^{\star} \boldsymbol{f}_{L}$ was shown in 3.3, and so $\boldsymbol{f}^{T} \boldsymbol{P}(\boldsymbol{X}) \boldsymbol{f}=0, f_{L}^{T} \boldsymbol{P}(\boldsymbol{X})_{L L}^{\star} f_{L}=0, \boldsymbol{f} \in \mathscr{N}(\boldsymbol{P}(\boldsymbol{X}))$, and $f_{L} \in \mathscr{N}\left(\boldsymbol{P}(\boldsymbol{X})_{L L}^{\star}\right)$ 
are all equivalent when $\boldsymbol{P}(\boldsymbol{X})_{U U} \boldsymbol{f}_{U}=-\boldsymbol{P}(\boldsymbol{X})_{U L} \boldsymbol{f}_{L}$. The proof concludes by noting that $\boldsymbol{P}(\boldsymbol{X})_{U U} \boldsymbol{f}_{U}=-\boldsymbol{P}(\boldsymbol{X})_{U L} \boldsymbol{f}_{L}$ always holds either by (" $\Leftarrow$ ") assumption or by (" $\Rightarrow$ ") its equivalence to $(\boldsymbol{P}(\boldsymbol{X}) \boldsymbol{f})_{U}=\overrightarrow{0}$ which follows from $\boldsymbol{f} \in \mathscr{N}(\boldsymbol{P}(\boldsymbol{X}))$.

For a general penalty $\boldsymbol{P}(\boldsymbol{X})$, taking $f_{L}$ to be the orthogonal projection of the residuals $\hat{\boldsymbol{\varepsilon}}_{L}$ onto $\mathscr{N}\left(\boldsymbol{P}(\boldsymbol{X})_{L L}^{\star}\right)$ is not penalized in $\sqrt{4.3}$, and if the components of $\boldsymbol{f}_{L}$ are constant within feature data manifolds, these constants get mapped to the unlabeled set $f_{U}$ accordingly. In other words, Proposition 2 and the above discussion establish that the safe semi-parametric estimator maps residuals properly from appropriate null spaces and adds flexibility over using responses directly.

\subsubsection{Existence and Uniqueness}

The implications of $\boldsymbol{P}(\boldsymbol{X})_{U U} \succ 0$ within Lemma 1 and Proposition 1 are investigated next. If instead $\boldsymbol{P}(\boldsymbol{X})_{U U} \succeq 0$, the optimal $\widehat{\boldsymbol{\beta}}$ and $\widehat{\boldsymbol{f}}_{L}$ from Proposition 1 are still unique, but each component of $\widehat{\boldsymbol{f}}_{U}$ is unique if and only if $\boldsymbol{P}(\boldsymbol{X})_{U U} \succ 0$ by 3.2 from within Lemma 1. A sufficient condition for when $\boldsymbol{P}(\boldsymbol{X})_{U U} \succ 0$ often boils down to every connected component in graph $\boldsymbol{W}(\boldsymbol{X})$ having a label or more precisely $\boldsymbol{P}(\boldsymbol{X})_{U U} \succ 0 \Longleftrightarrow L \cap \omega_{i} \neq \emptyset \forall i$ from Section 3.3.1. While the precise interpretation depends on the exact choice of $\boldsymbol{P}(\boldsymbol{X})$, Proposition 3 provides a general sufficient condition for $\boldsymbol{P}(\boldsymbol{X})_{U U} \succ 0$ that often reduces to this intuitive interpretation.

Proposition 3. If $\boldsymbol{P}(\boldsymbol{X}) \succeq 0$ and $\boldsymbol{P}(\boldsymbol{X})_{L U} \boldsymbol{v}_{U} \neq \overrightarrow{0} \forall$ nonzero $\boldsymbol{v}_{U} \in \mathbb{R}^{|U|}$, then $\boldsymbol{P}(\boldsymbol{X})_{U U} \succ 0$.

Proof. We use the matrix result: if $\boldsymbol{P}(\boldsymbol{X}) \succeq 0$, then $\boldsymbol{v}^{T} \boldsymbol{P}(\boldsymbol{X}) \boldsymbol{v}=0 \Longleftrightarrow \boldsymbol{v} \in \mathscr{N}(\boldsymbol{P}(\boldsymbol{X}))$ for any $\boldsymbol{v} \in \mathbb{R}^{n}$. If $\boldsymbol{P}(\boldsymbol{X})_{U U} \boldsymbol{v}_{U}=\overrightarrow{0}$, then $\boldsymbol{P}(\boldsymbol{X})_{L U} \boldsymbol{v}_{U}=\overrightarrow{0}$ follows when $\boldsymbol{v}_{L}=\overrightarrow{0}$. By contrapositive, if $\mathscr{N}\left(\boldsymbol{P}(\boldsymbol{X})_{L U}\right)=\{\overrightarrow{0}\}$, then $\mathscr{N}\left(\boldsymbol{P}(\boldsymbol{X})_{U U}\right)=\{\overrightarrow{0}\}$.

In general, the sufficient condition for uniqueness from Proposition 3 involving $\boldsymbol{P}(\boldsymbol{X})_{L U}$ is not necessary, e.g., just take a procedure that automatically sets $\boldsymbol{P}(\boldsymbol{X})_{L U}=\mathbf{0}$ such as 
$\boldsymbol{P}(\boldsymbol{X})=\boldsymbol{I}$. On the other hand, this sufficient condition is necessary for some choices of $\boldsymbol{P}(\boldsymbol{X})$ including the Laplacian and normalized Laplacian. With such choices of $\boldsymbol{P}(\boldsymbol{X})$, this necessary and sufficient condition establishes that every unlabeled case must somehow be "related" to a labeled case in order for $\widehat{\boldsymbol{f}}_{U}$ to be unique.

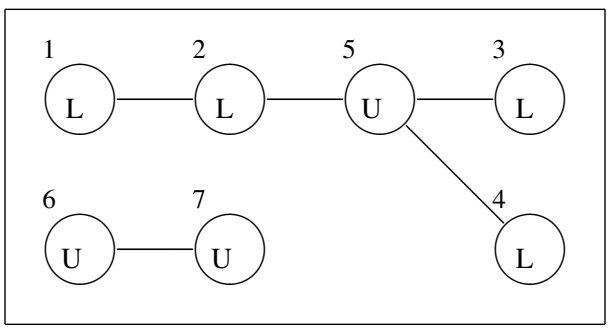

The graph $\boldsymbol{W}(\boldsymbol{X})$ on left with $n=7$ vertices and binary weighted edges is used to illustrate some general concepts on the topic of $\boldsymbol{P}(\boldsymbol{X})_{U U} \succ 0$. If $\boldsymbol{P}(\boldsymbol{X})=\boldsymbol{\Delta}(\boldsymbol{X})$ is the Laplacian of the full graph $\boldsymbol{W}(\boldsymbol{X})$ and $\boldsymbol{P}\left(\boldsymbol{X}_{U}\right)=\boldsymbol{\Delta}\left(\boldsymbol{X}_{U}\right)$ is the Laplacian of the corresponding unlabeled subgraph $\boldsymbol{W}(\boldsymbol{X})_{U U}$, then

$$
\begin{aligned}
& 5 \quad 6 \quad 7 \\
& \underline{5} \underline{6} \underline{7} \quad \underline{5} \quad \underline{6} \underline{7} \\
& \mathscr{N}\left(\boldsymbol{\Delta}(\boldsymbol{X})_{U U}\right)=\operatorname{span}\left\{(0,1,1)^{T}\right\} \subset \operatorname{span}\left\{(1,0,0)^{T},(0,1,1)^{T}\right\}=\mathscr{N}\left(\boldsymbol{\Delta}\left(\boldsymbol{X}_{U}\right)\right) .
\end{aligned}
$$

The provided basis for $\mathscr{N}\left(\boldsymbol{\Delta}\left(\boldsymbol{X}_{U}\right)\right)$ is based on the partition $\{\{5\},\{6,7\}\}$ of $U=\{5,6,7\}$ by the connected components of the subgraph graph $\boldsymbol{W}(\boldsymbol{X})_{U U}$, whereas that for $\mathscr{N}\left(\boldsymbol{\Delta}(\boldsymbol{X})_{U U}\right)$ effectively removes basis vector $(1,0,0)^{T}$ corresponding to subset $\{5\} \subseteq U$ due to its connections to $L=\{1,2,3,4\}$ in the full graph $\boldsymbol{W}(\boldsymbol{X})$. The purely unlabeled connected component $\{6,7\} \subset U$ accounts for the nonzero vectors in $\mathscr{N}\left(\boldsymbol{\Delta}(\boldsymbol{X})_{U U}\right)$ and is the sole reason that $\boldsymbol{P}(\boldsymbol{X})_{U U}=\boldsymbol{\Delta}(\boldsymbol{X})_{U U} \succeq 0$. Equivalently, the corresponding basis vector $(0,1,1)^{T}$ fails the condition from Proposition 3 with $\boldsymbol{\Delta}(\boldsymbol{X})_{L U}(0,1,1)^{T}=\overrightarrow{0}$, since the last two columns of $\boldsymbol{\Delta}(\boldsymbol{X})_{L U}=-\boldsymbol{W}(\boldsymbol{X})_{L U}$ are all zeros. Thus, if $(\widehat{\boldsymbol{f}}, \widehat{\boldsymbol{\beta}})$ is a solution to 3.1 , then adding any multiple of $(0,1,1)^{T}$ to $\widehat{\boldsymbol{f}}_{U}$ produces another solution, and only the components of an optimal $\widehat{f}_{U}$ from 3.1 corresponding to vertices $\{6,7\}$ are arbitrary in this manner. In terms of the condition from Proposition 3, it was only necessary for the two basis vectors $\boldsymbol{v}_{U} \in \mathscr{N}\left(\boldsymbol{\Delta}\left(\boldsymbol{X}_{U}\right)\right)$ from 3.5 to pass the check $\boldsymbol{\Delta}(\boldsymbol{X})_{L U} \boldsymbol{v}_{U} \neq \overrightarrow{0}$ in order to show that $\boldsymbol{\Delta}(\boldsymbol{X})_{U U} \succ 0$ because $\mathscr{N}\left(\boldsymbol{\Delta}(\boldsymbol{X})_{U U}\right) \subseteq \mathscr{N}\left(\boldsymbol{\Delta}\left(\boldsymbol{X}_{U}\right)\right)$. 
Unfortunately, the requirement $\boldsymbol{P}(\boldsymbol{X})_{U U} \succ 0$ for either the Laplacian or normalized Laplacian may be more difficult to satisfy if $|U| / n \rightarrow 1$ as $n \rightarrow \infty$. In this limit, especially if the graphing procedure constrains the number of edges as a function of $n$, the minimum eigenvalue of $\boldsymbol{P}(\boldsymbol{X})_{U U}$ goes to zero as purely unlabeled connected components become more probable. These types of issues are directly addressed with the regularized procedure presented in next Section 3.4.

\subsection{Joint Semi-Parametric Prediction}

The labeled loss optimization problem (3.1) provides a safe semi-supervised semi-parametric graph-based approach. To capitalize on the cluster assumption results from Section 3.3.1 and optimize the bias-variance trade-off, we favor sparse Gram matrices $\boldsymbol{W}(\boldsymbol{X})$, and this motivates our later preprocessing of a sparse $\boldsymbol{W}(\boldsymbol{X})$ with a $k$-NN graph. However, such preprocessing is in conflict with $\boldsymbol{P}(\boldsymbol{X})_{U U} \succ 0$ from Section 3.3.2 and hence the uniqueness of optimal $f_{U}$ by (3.2). Also, even if the required inverse is unique, the approach may still be computationally unstable due to very small nonzero eigenvalues. All these issues are circumvented with the joint optimization

$$
\min _{\boldsymbol{f}, \boldsymbol{\beta}, \boldsymbol{Y}_{U}}\left\{\sum_{i=1}^{n} c_{i}\left(\boldsymbol{Y}_{i}\left(\boldsymbol{Y}_{U}\right)-\boldsymbol{f}_{i}-\boldsymbol{x}_{i}^{T} \boldsymbol{\beta}\right)^{2}+\lambda_{1} f^{T} \boldsymbol{P}(\boldsymbol{X}) \boldsymbol{f}+\lambda_{2} \boldsymbol{\beta}^{T} \boldsymbol{\beta}+\gamma \boldsymbol{Y}_{U}^{T} \boldsymbol{Y}_{U}\right\},
$$

where $\left\{c_{i}>0\right\}_{i=1}^{n}$. The safe semi-supervised semi-parametric model (S4PM) has predictions $\widehat{\boldsymbol{\eta}}=\boldsymbol{X} \widehat{\boldsymbol{\beta}}+\widehat{\boldsymbol{f}}$ based on the solution to 3.6 given in Proposition 4

Proposition 4. Define the $n \times n$ diagonal matrix $(\boldsymbol{C})_{i i}=c_{i}-\left(\frac{c_{i}^{2}}{c_{i}+\gamma}\right) \mathscr{I}_{\{i \in U\}}$ from the indicator function $\mathscr{I}_{\{\cdot\}}$ and matrices $\boldsymbol{M}(\boldsymbol{X})=\left(\boldsymbol{C}+\lambda_{1} \boldsymbol{P}(\boldsymbol{X})\right)^{-1} \boldsymbol{C}$ and $\tilde{\boldsymbol{M}}(\boldsymbol{X})=\boldsymbol{C}(\boldsymbol{I}-\boldsymbol{M}(\boldsymbol{X}))$. If $\lambda_{2}>0$ or $\operatorname{rank}\left(\boldsymbol{X}_{L}\right)=p$ and $\gamma>0$ or $\boldsymbol{P}(\boldsymbol{X})_{U U} \succ 0$, then the unique solution $\left(\widehat{\boldsymbol{f}}, \widehat{\boldsymbol{\beta}}_{\hat{\boldsymbol{Y}}} \widehat{\boldsymbol{Y}}_{U}\right)$ to 
optimization (3.6) is given by

$$
\begin{aligned}
\widehat{\boldsymbol{\beta}} & =\left(\boldsymbol{X}^{T} \tilde{\boldsymbol{M}}(\boldsymbol{X}) \boldsymbol{X}+\lambda_{2} \boldsymbol{I}\right)^{-1} \times \boldsymbol{X}^{T} \tilde{\boldsymbol{M}}(\boldsymbol{X}) \boldsymbol{Y}(\overrightarrow{0}) \\
\widehat{\boldsymbol{f}} & =\boldsymbol{M}(\boldsymbol{X})(\boldsymbol{Y}(\overrightarrow{0})-\boldsymbol{X} \widehat{\boldsymbol{\beta}}) \\
\widehat{\boldsymbol{Y}}_{U} & =\frac{1}{\gamma} \boldsymbol{C}_{U U} \widehat{\boldsymbol{\eta}}_{U} .
\end{aligned}
$$

Proof. Define the $|U| \times|U|$ diagonal matrix $\boldsymbol{V}_{U U}$ such that $\left(\boldsymbol{V}_{U U}\right)_{i i}=c_{i}$. The $\boldsymbol{Y}_{U}$-score yields $\widehat{\boldsymbol{Y}}_{U}=\left(\boldsymbol{V}_{U U}+\gamma \boldsymbol{I}\right)^{-1} \boldsymbol{V}_{U U} \widehat{\boldsymbol{\eta}}_{U}$ and identity

$$
\boldsymbol{V}_{U U}\left(\widehat{\boldsymbol{Y}}_{U}-\widehat{\boldsymbol{\eta}}_{U}\right)=\boldsymbol{C}_{U U} \widehat{\boldsymbol{\eta}}_{U}
$$

follows. Identity 3.7 simplifies the $\boldsymbol{f}$-score $-\boldsymbol{C}(\boldsymbol{Y}(\overrightarrow{0})-\widehat{\boldsymbol{\eta}})+\lambda_{1} \boldsymbol{P}(\boldsymbol{X}) \widehat{\boldsymbol{f}}=\overrightarrow{0}$ to

$$
\left(\boldsymbol{C}+\lambda_{1} \boldsymbol{P}(\boldsymbol{X})\right) \widehat{\boldsymbol{f}}=\boldsymbol{C}(\boldsymbol{Y}(\overrightarrow{0})-\boldsymbol{X} \widehat{\boldsymbol{\beta}})
$$

Identity 3.77 also simplifies the $\boldsymbol{\beta}$-score $-\boldsymbol{X}^{T} \boldsymbol{C}(\boldsymbol{Y}(\overrightarrow{0})-\widehat{\boldsymbol{\eta}})+\lambda_{2} \widehat{\boldsymbol{\beta}}=\overrightarrow{0}$ to

$$
\left(\boldsymbol{X}^{T} \tilde{\boldsymbol{M}}(\boldsymbol{X}) \boldsymbol{X}+\lambda_{2} \boldsymbol{I}\right) \widehat{\boldsymbol{\beta}}=\boldsymbol{X}^{T} \tilde{\boldsymbol{M}}(\boldsymbol{X}) \boldsymbol{Y}(\overrightarrow{0})
$$

The $\gamma=0$ case of optimization $(3.6)$ is the solution to labeled loss $(3.1)$ since $C_{U U}=0 \boldsymbol{I}$. When $\gamma=\infty$, problem 3.6 reduces to

$$
\min _{\boldsymbol{f}, \boldsymbol{\beta}}\left\{\sum_{i=1}^{n} c_{i}\left(\boldsymbol{Y}_{i}(\overrightarrow{0})-\boldsymbol{f}_{i}-\boldsymbol{x}_{i}^{T} \boldsymbol{\beta}\right)^{2}+\lambda_{1} \boldsymbol{f}^{T} \boldsymbol{P}(\boldsymbol{X}) \boldsymbol{f}+\lambda_{2} \boldsymbol{\beta}^{T} \boldsymbol{\beta}\right\}
$$

and shrinks unlabeled predictions $\widehat{\boldsymbol{f}}_{U}+\boldsymbol{X}_{U} \widehat{\boldsymbol{\beta}}$ towards $\overrightarrow{0}$. This is justifiable when there are unlabeled extrapolations and smaller prediction variance is desirable. Similar to labeled loss 


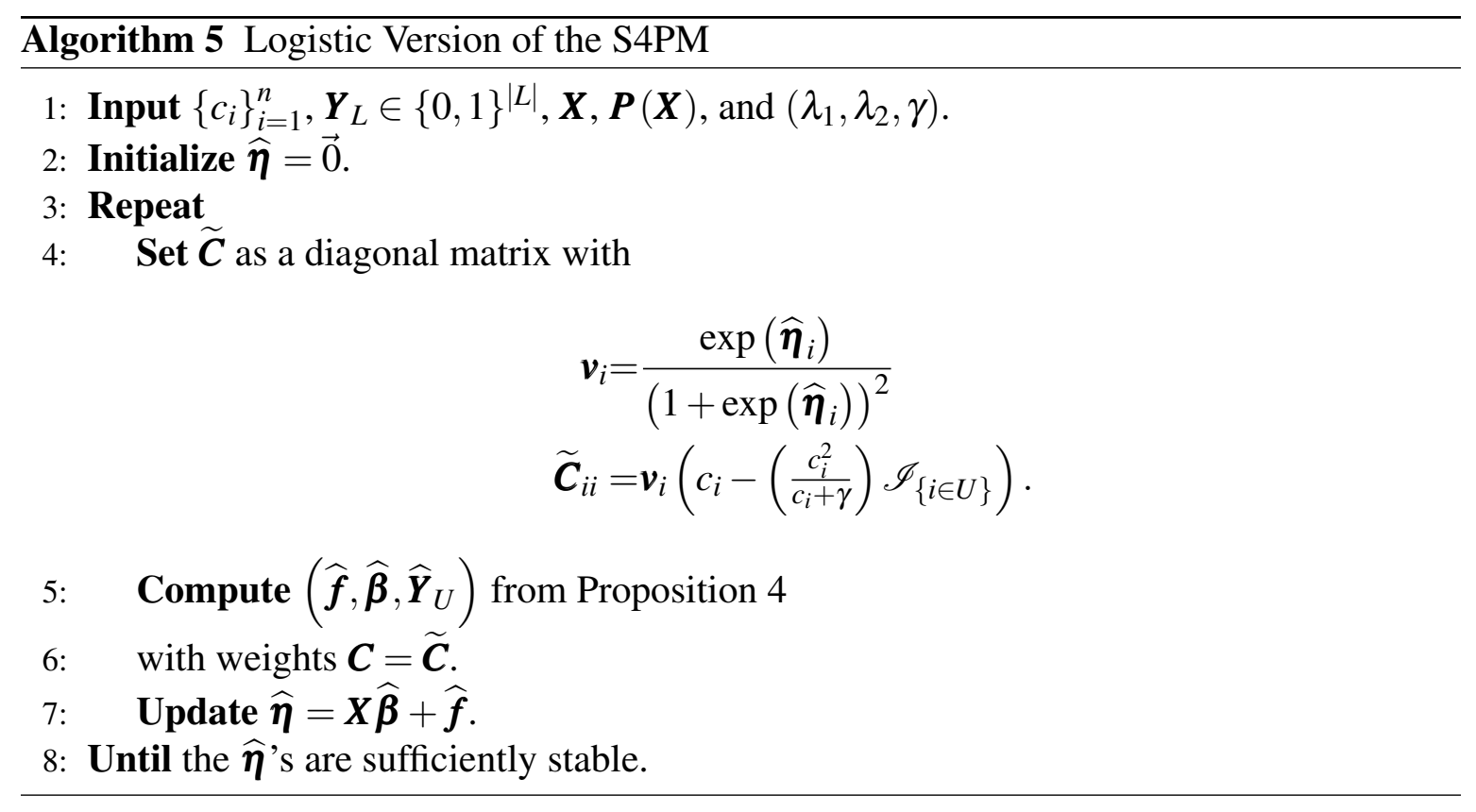

(3.1) other convex loss functions also follow in the natural way. For example, the logistic version of the S4PM classifications $\mathbf{1}_{\{\widehat{\boldsymbol{\eta}}>0\}}$ from Algorithm 6 uses the weighted variance matrix $\widetilde{C}$ in place of the matrix $\boldsymbol{C}$. Algorithm 6 is used to fit the classification data sets in the challenging Section 3.5 benchmarks.

\subsubsection{Joint Laplacian and Joint Spreading Derivatives}

Our contention is that several ("purely semi-supervised") Laplacian or normalized Laplacian graph-based techniques in the literature are variants of the joint optimization problem

$$
\min _{\boldsymbol{f}, \boldsymbol{Y}_{U}}\left\{\sum_{i=1}^{n} c_{i}\left(\boldsymbol{Y}_{i}\left(\boldsymbol{Y}_{U}\right)-\boldsymbol{f}_{i}\right)^{2}+\lambda \boldsymbol{f}^{T} \boldsymbol{P}(\boldsymbol{X}) \boldsymbol{f}+\gamma \boldsymbol{Y}_{U}^{T} \boldsymbol{Y}_{U}\right\}
$$

although they are rarely presented in this manner. This conclusion was drawn from an exhaustive search of the literature on this topic. Pointing out these connections is not in and of itself a novelty of this work, but it does provide a succinct and useful presentation of our work and demonstrates the generality of our novel safe generalization in optimization (3.6). 
When $\boldsymbol{P}(\boldsymbol{X})=\boldsymbol{\Delta}(\boldsymbol{X})$ or $\boldsymbol{P}(\boldsymbol{X})=\widetilde{\Delta}(\boldsymbol{X})$, some well-known, purely semi-supervised graphbased approaches were first defined as the limit of a convergent iterative algorithm, while this limit is also the solution to a particular optimization problem. For example, label spreading to our knowledge was the first of such approaches with the normalized Laplacian (Chapelle et al. 2006c, Chapter 11). Their iterative algorithm converges to

$$
\begin{aligned}
\widehat{\boldsymbol{f}} & =(1-\alpha)(\boldsymbol{I}-\alpha(\boldsymbol{I}-\widetilde{\boldsymbol{\Delta}}(\boldsymbol{X})))^{-1} \boldsymbol{Y}(\overrightarrow{0}) \\
& =(\boldsymbol{I}+\lambda \widetilde{\boldsymbol{\Delta}}(\boldsymbol{X}))^{-1} \boldsymbol{Y}(\overrightarrow{0})
\end{aligned}
$$

with tuning parameter $\alpha=\frac{\lambda}{\lambda+1} \in[0,1)$, and this $\widehat{\boldsymbol{f}}$ is the solution to

$$
\min _{\boldsymbol{f}} \sum_{i=1}^{n}\left(\boldsymbol{Y}_{i}(\overrightarrow{0})-\boldsymbol{f}_{i}\right)^{2}+\lambda \boldsymbol{f}^{T} \widetilde{\boldsymbol{\Delta}}(\boldsymbol{X}) \boldsymbol{f}
$$

i.e., a special case of 3.8 with $\boldsymbol{P}(\boldsymbol{X})=\widetilde{\boldsymbol{\Delta}}(\boldsymbol{X})$ and $\gamma=\infty$. As a second example, a diffusion kernel approach (Zhou et al., 2004a) results from the normalized Laplacian optimization with labeled loss

$$
\min _{\boldsymbol{f}} \sum_{i=1}^{n}\left(\boldsymbol{Y}_{i}\left(\boldsymbol{f}_{U}\right)-\boldsymbol{f}_{i}\right)^{2}+\lambda \boldsymbol{f}^{T} \widetilde{\boldsymbol{\Delta}}(\boldsymbol{X}) \boldsymbol{f}
$$

i.e., another special case of 3.8 with $\gamma=0$ and $\boldsymbol{Y}_{U}=\boldsymbol{f}_{U}$. Regularized Laplacian approaches can also be motivated as special cases of 3.8 using the Laplacian $\boldsymbol{\Delta}(\boldsymbol{X})$ in place of the normalized Laplacian (Belkin et al., 2006, Chapelle et al., 2006c, Chapter 11). For this work, we represent this class of purely semi-supervised approaches by optimizing (3.8) with the Laplacian (Joint Laplacian) or the normalized Laplacian (Joint Spreading). We also provide an efficient implementation that estimates $(h, \lambda, \gamma)$ with cross-validation (refer to Section 3.5.

Not all relevant semi-supervised graph-based techniques are covered by optimization 


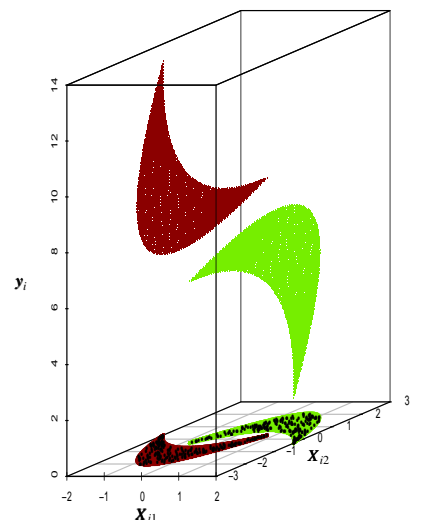

(a)
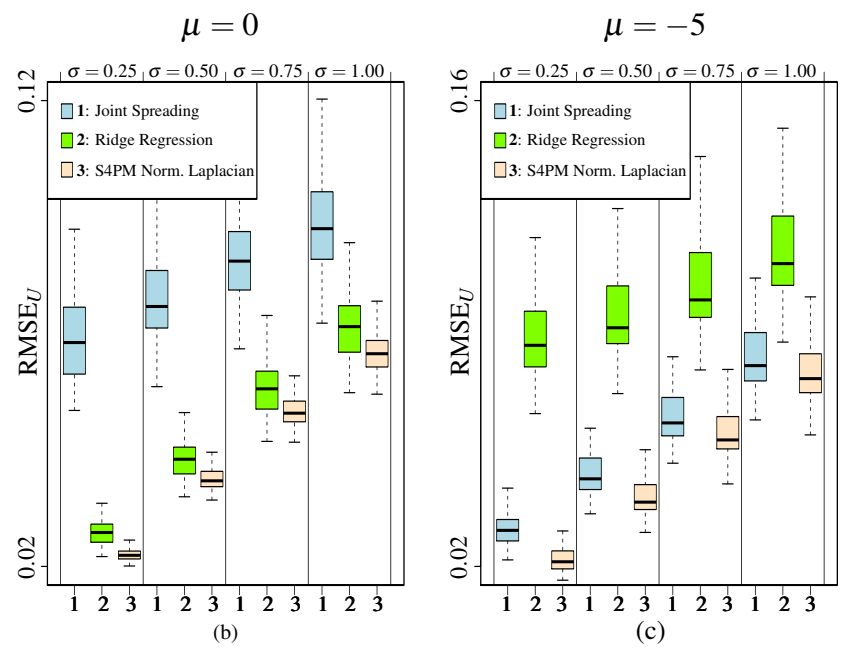

Figure 3.1: Simulation Results for the "Two Moons" Regression Example. (a) A 3D Plot of the True Response Surface with its 2D Shadow on the Feature Space. Unlabeled RMSE: (b) $\mu=0$ and (c) $\mu=-5$.

3.8. An obvious example is the Label Propagation algorithm: initialize $\widehat{f}=\overrightarrow{0}$ and iterate $\widehat{\boldsymbol{f}}=\boldsymbol{S} \boldsymbol{Y}\left(\widehat{\boldsymbol{f}}_{U}\right)$ with $\boldsymbol{S}=(\boldsymbol{W}(\boldsymbol{X})+\boldsymbol{\Delta}(\boldsymbol{X}))^{-1} \boldsymbol{W}(\boldsymbol{X})$ to obtain an estimate $\widehat{\boldsymbol{f}}$ of a harmonic function 11 However, Culp and Ryan (2013) and Culp et al. (2009) related such harmonic function approaches to a joint optimization problem that is similar to 3.8 ) as well as the self-training class of semi-supervised algorithms. Viable prediction methodology for the labeled and unlabeled parts of $\widehat{\boldsymbol{f}}$ are given in these other works. This literature discussion provides considerations for graph-based semi-supervised techniques directly related to this work and is not exhaustive of all such techniques, e.g., the connection to the S3VM and (3.8) is given in Culp and Ryan (2013).

\footnotetext{
${ }^{1}$ The original Label Propagation algorithm (mistakenly) lists its steps as (i) $\widehat{\boldsymbol{f}}=\widehat{\boldsymbol{S f}}$ and (ii) $\widehat{\boldsymbol{f}}_{L}=\boldsymbol{Y}_{L}$ and is hence not equivalent because the labeled predictions are clamped $\widehat{\boldsymbol{f}}_{L}=\boldsymbol{Y}_{L}$ in the last call. But if $\boldsymbol{X}_{i}=\boldsymbol{X}_{j}$ for some $i, j \in L$, then clamping $\widehat{\boldsymbol{f}}_{L}=\boldsymbol{Y}_{L}$ maps two equal covariate vectors to distinct predictions when $\boldsymbol{y}_{i} \neq \boldsymbol{y}_{j}$. Instead just switch the order of the steps and iterate (i) $\widehat{\boldsymbol{f}}_{L}=\boldsymbol{Y}_{L}$ and (ii) $\widehat{\boldsymbol{f}}=\boldsymbol{S} \widehat{\boldsymbol{f}}$ until convergence to get labeled predictions (Culp et al., 2009).
} 


\subsection{Results}

The competitiveness of the safe semi-supervised semi-parametric modeling (S4PM) approach is illustrated in the simulated and real data prediction scenarios of Sections 3.5 .1 and 4.6.2. Two versions of the S4PM 3.6 were fit: (i) $\boldsymbol{P}(\boldsymbol{X})$ was set to the Laplacian and (ii) $\boldsymbol{P}(\boldsymbol{X})$ was set to the normalized Laplacian. The corresponding pure semi-supervised comparisons were made to both (i) Joint Laplacian and (ii) Joint Spreading. The efficient semi-supervised Anchor graph approach (Liu et al., 2010) was also fit for comparisons; its ridge tuning parameter was estimated by cross-validation while its graph parameters were set at $s=5$ and $c_{n}=4$. For supervised comparisons, ridge regression was fit with the glmnet package in $\mathrm{R}$ at its default cross-validation settings (Friedman et al., 2010), and the supervised SVM was fit using the radial basis function optimization setting within the caret package (Kuhn, 2014). The response and feature data were mean centered and scaled to unit variance using the mean and variance of the labeled data.

Parameters tuned either the graph itself or the penalties. A $k=6$ nearest neighbor proximity graph $\boldsymbol{W}(\boldsymbol{X})$ was fit to each data set using the cosine distance metric in the following manner. For each $i \in L \cup U$, initialize $\boldsymbol{d}_{i}$ to the $n \times 1$ cosine distance vector between $\boldsymbol{x}_{i}$ and each $\boldsymbol{x}_{j}$ with $j \in L \cup U$ and then set the components of $d_{i}$ to infinity, except for the $k+1$ smallest distances. Next, set $\boldsymbol{d}_{i j}=\min \left\{\boldsymbol{d}_{i j}, \boldsymbol{d}_{j i}\right\}$ for all $i, j$ to preserve symmetry. The $i$ th row of $\boldsymbol{W}(\boldsymbol{X})$ is the local kernel function $K_{h}(x)=\exp (-x / h)$ applied to $\boldsymbol{d}_{i}$ for each $i \in L \cup U$ with bandwidth parameter $h>0$. Lagrangian parameters $\lambda_{1}$ and $\lambda_{2}$ control the relative importance of the respective graph and linear terms, and parameter $\gamma$ controls the stability of the semi-supervised approach. Our experience suggests that performance is robust to the choice of $k$ as long as it is relatively small.

Much work on computational efficiency was done to fit the semi-supervised technique quickly in memory. Since this approach is based on symmetric matrices, a Cholesky de- 
composition and efficient storage of matrices (e.g., store the upper triangle of $\boldsymbol{P}(\boldsymbol{X})$ and the lower triangle of $\boldsymbol{M}(\boldsymbol{X})$ ) in conjunction with optimized LAPACK/ATLAS C-routines boosted speed substantially. An even larger speedup was due to the following, novel Stagewise Cross Validation (SCV) scheme. For each $h$, adjacencies in the graph

$$
\boldsymbol{W}(\boldsymbol{X})_{L L}+\boldsymbol{\Delta}(\boldsymbol{X})_{L U} \boldsymbol{\Delta}(\boldsymbol{X})_{U U}^{-1} \boldsymbol{\Delta}(\boldsymbol{X})_{U L}
$$

on $L$ were computed to quantify labeled-to-labeled connectives through labeled and unlabeled networks in the larger graph $\boldsymbol{W}(\boldsymbol{X})$ on $L \cup U$ (Culp and Ryan, 2013). Four parameters $h, \lambda_{1}, \lambda_{2}, \gamma$ were then estimated with 3 -fold CV from graph 3.8 and data $\boldsymbol{X}_{L}$. (While $\boldsymbol{X}_{L}$ played the role of $\boldsymbol{X}$ during the fitting of a given fold, the responses in the other 2 folds were omitted and played the role of $Y_{U}$.) Lastly, parameter $\gamma$ was re-estimated with respect to the full graph $\boldsymbol{W}(\boldsymbol{X})$ with fixed, optimal values for $h, \lambda_{1}, \lambda_{2}$ to stabilize the predictions. A comparison is later given to demonstrate that the performance of SCV and ordinary CV were about the same while computational times favored SCV.

\subsubsection{Semi-Supervised Smoothness in Regression}

The regression-based version of the "two moons" example in Figure 3.1 is used to illustrate the method with simulated data sets. Each row $x_{i}$ of the $400 \times 2$ feature data matrix $\boldsymbol{X}$ is within a moon of the $y=0$ plane of Figure 3.1.(a). Responses

$$
\boldsymbol{Y} \sim \mathrm{N}\left(10 \times \overrightarrow{1}+\mu z+\boldsymbol{X}\left(\begin{array}{r}
-1.5 \\
1.5
\end{array}\right), \sigma^{2} \boldsymbol{I}\right)
$$

where $z_{i}=\mathscr{I}_{\left\{x_{i} \in \text { moon } 1\right\}}$. Vector $\boldsymbol{z} \in\{0,1\}^{400}$, which codes the two clusters of feature data, is not in the column space of $\boldsymbol{X}$. The scalar parameter $\mu$ calibrates the semi-supervised smoothness assumption: (i) $\mu=0$ (knowing the moons has no value) and (ii) $\mu=-5$ (know- 

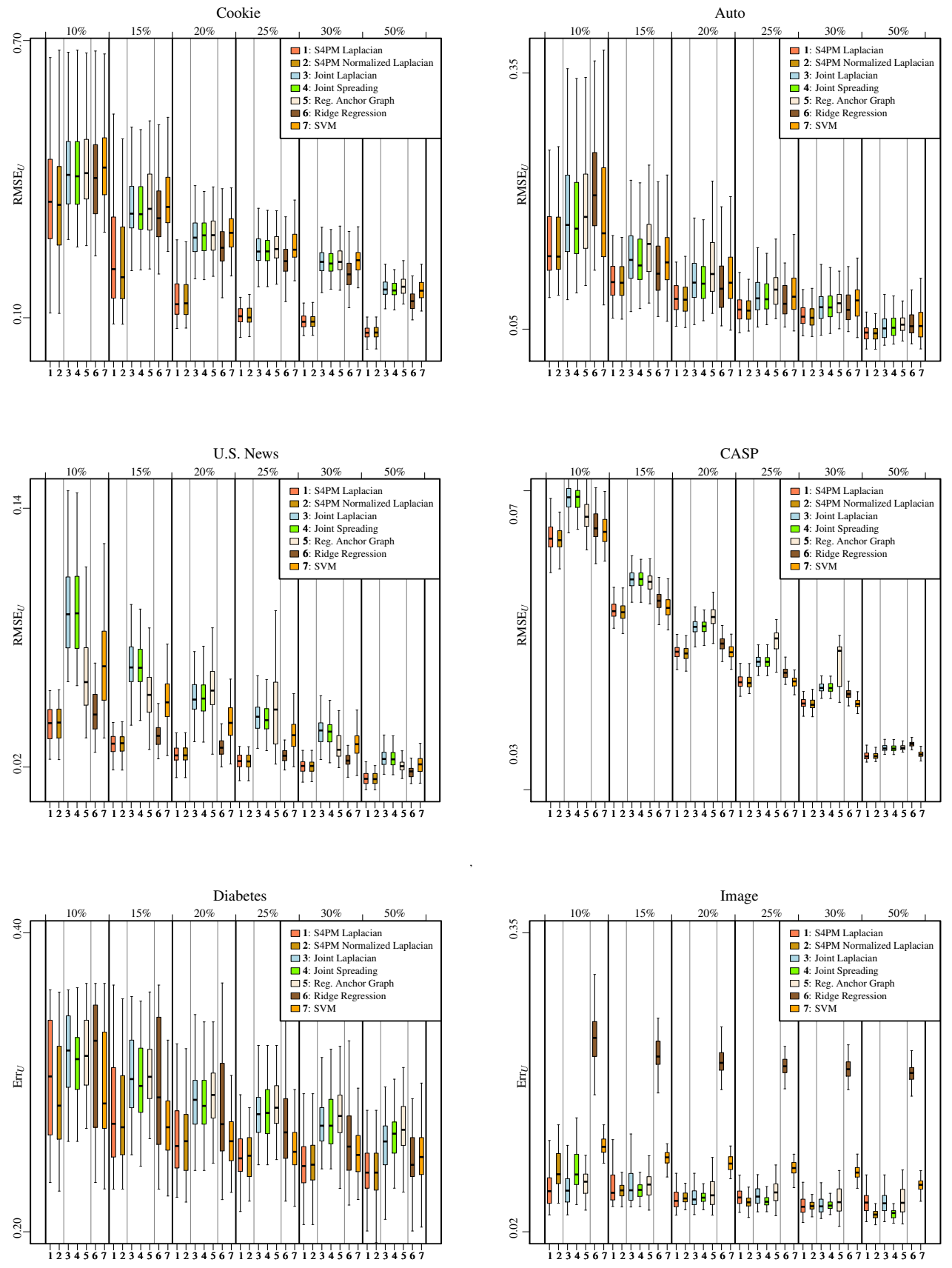

Figure 3.2: Unlabeled Performance on Publicly Available Data Sets: Regression (top two rows) and Classification (bottom row). The labeled sets were selected at random with labeled percentages $100|L| / n=10,15,20,25,30,50 \%$. Performance measures are unlabeled RMSE in the regression examples and unlabeled error rate in the classification examples. 
Table 3.1: Sampling was used to fit the CASP data. The data were partitioned at random into sets $L, U_{1}, U_{2}$ with $|L|=1,000$. Set $U_{1}$ was the unlabeled set used during training. Table entries are averages of fifty partitioning at each $\left|U_{1}\right|$ of composite unlabeled RMSE over $U=U_{1} \cup U_{2}$ for the continuous response and runtime (in minutes).

\begin{tabular}{lcccccc}
\hline \hline$\left|U_{1}\right|$ & 100 & 500 & 1,000 & 3,000 & 5,000 & 44,730 \\
\hline $\operatorname{RMSE}_{U}$ & 0.026 & 0.026 & 0.026 & 0.026 & 0.026 & kill -9 error \\
Time & 0.351 & 0.468 & 0.519 & 1.037 & 2.028 & $240+$ \\
\hline
\end{tabular}

ing the moons has value). The $3 \mathrm{D}$ response surface (ii) $\mu=-5$ is the one plotted in Figure 3.1 (a). Throughout this simulation study, $|L|=50$ observations were randomly labeled at a given $\sigma \in\{0.25,0.5,0.75,1.00\}$. This process was repeated 100 times at each combination of $\mu, \sigma$. For each simulated data set, the unlabeled root mean squared error $\left(\mathrm{RMSE}_{U}\right)$ was computed for three approaches: Joint Spreading, Ridge Regression, and the Normalized Laplacian version of the S4PM. Panels (b) and (c) of Figure 3.1 summarize the results when $\mu=0$ and $\mu=-5$. Ridge regression works well when the semi-supervised assumption is misspecified (i.e., $\mu=0$ ), but struggles when it is satisfied (i.e., $\mu=-5$ ). Joint Spreading, on the other hand, struggles in the case of $\mu=0$ because it favors $\boldsymbol{\eta}=\boldsymbol{f} \in \mathscr{N}(\widetilde{\boldsymbol{\Delta}}(\boldsymbol{X}))$ that emphasize the two moons even when they have no predictive value. The S4PM works well in either case. The non-parametric fit gives it an advantage over ridge regression when $\mu=0$, and the linear fit component gives it an advantage over Joint Spreading when $\mu=-5$.

\subsubsection{Real Comparisons}

Regression tests were performed on four challenging regression data sets with a pre-defined, continuous response: the U.S. News data (Ryan and Culp, 2015) and the Cookie, Auto, and Physicochemical Properties of Protein Tertiary Structure (CASP) data sets (Lichman, 2013). With the exception of CASP, all data scaling and preprocessing are explained in Ryan and Culp (2015).

In order to quickly fit the larger $45,730 \times 9$ CASP data, we first explore a sampling 
concept and randomly partition the unlabeled data $U$ into two sets: $U_{1}, U_{2}$. Observations $U_{1}$ were used as the unlabeled data for fitting $\widehat{\boldsymbol{\eta}}$, while observations $U_{2}$ were set aside for prediction. Prediction of the responses on $U_{1}\left(\right.$ on $U_{2}$ ) is sometimes called transductive (outof-sample) prediction. Unlike transduction with predictions $\widehat{\boldsymbol{\eta}}_{U_{1}}$ on $U_{1}$, our procedure does not lend itself to an obvious out-of-sample prediction rule for an observation in $U_{2}$ with feature vector $\boldsymbol{x}_{0}$, but out-of-sample prediction is always possible with continuous feature data by interpolating the predictions $\widehat{\boldsymbol{\eta}}$ on $L \cup U_{1}$. To this end, recall that $\boldsymbol{W}(\boldsymbol{X})$ is the Gram matrix of local kernel $K_{h}\left(\boldsymbol{x}_{i}, \boldsymbol{x}_{j}\right)$ and let $\boldsymbol{W}(X)_{i 0}=K_{h}\left(\boldsymbol{x}_{i}, \boldsymbol{x}_{0}\right)$ for $i \in L \cup U_{1}$. The interpolation function

$$
\widehat{\boldsymbol{\eta}}\left(\boldsymbol{x}_{0}\right)=\boldsymbol{x}_{0}^{T} \widehat{\boldsymbol{\beta}}+\frac{\sum_{i} a_{i} \widehat{\boldsymbol{f}}_{i}}{\sum_{i} a_{i}} \text { with } a_{i}=\frac{\boldsymbol{W}(\boldsymbol{X})_{i 0}}{1-\boldsymbol{W}(\boldsymbol{X})_{i 0}}
$$

was used to make the out-of-sample predictions on $U_{2}$. The performance and times in Table 3.1 justify this sampling approach for the CASP data set. The approach was beyond our computational reach when $\left|U_{1}\right|=44,730$ and $U_{2}=\emptyset$, but choices with $\left|U_{1}\right| \leq 1,000$ gave good performance in under a minute.

Results in Figure 3.2 were based on 100 randomly selected labeled sets at each labeled percentage $100|L| / n=10,15,20,25,30,50 \%$. We fixed $n=\left|L \cup U_{1}\right|=2,000$ for the CASP data and then predicted all the cases $U=U_{1} \cup U_{2}$. The three pure semi-supervised techniques, i.e., Joint Laplacian, Joint Spreading, and Regularized Anchor Graph, also used $n=2,000$ in this manner with the CASP data. Partitioning $U$ into $U_{1}, U_{2}$ was not needed for the other data sets. Both versions of our S4PM dominant the pure supervised and semi-supervised extremes across the four regression examples.

Algorithm 6 was used to predict the pre-defined, binary outcomes in two UCI classification examples: Diabetes and Images (Lichman, 2013). The results are also provided in Figure 3.2. For the Images data, there is no substantial improvement over the purely semi- 
Table 3.2: Average performance and computation time in minutes with $95 \%$ margins of error for publicly available data sets with $15 \%$ labeled sets selected at random. Performance measures are unlabeled RMSE in the regression examples with a continuous response (first four rows) and the unlabeled error rate in the classifications examples with a binary response (last two rows).

\begin{tabular}{|c|c|c|c|c|c|}
\hline Data & $n$ & $\overline{p p}$ & & SCV & $\overline{\mathrm{CV}}$ \\
\hline \multirow[t]{2}{*}{ Cookie } & 72 & 700 & $\operatorname{RMSE}_{U}$ & $1.397 \pm 0.061$ & $1.400 \pm 0.064$ \\
\hline & & & Time & $0.125 \pm 0.001$ & $0.159 \pm 0.001$ \\
\hline \multirow[t]{2}{*}{ Auto } & 205 & 24 & $\operatorname{RMSE}_{U}$ & $0.342 \pm 0.014$ & $0.337 \pm 0.013$ \\
\hline & & & Time & $0.003 \pm 0.001$ & $0.022 \pm 0.001$ \\
\hline \multirow[t]{2}{*}{ U.S. News } & 1,004 & 19 & $\operatorname{RMSE}_{U}$ & $0.110 \pm 0.004$ & $0.110 \pm 0.004$ \\
\hline & & & Time & $0.026 \pm 0.001$ & $0.332 \pm 0.001$ \\
\hline \multirow[t]{2}{*}{ CASP } & 45,730 & 9 & $\mathrm{RMSE}_{U}$ & $0.050 \pm 0.001$ & $0.050 \pm 0.001$ \\
\hline & & & Time & $0.181 \pm 0.001$ & $1.496 \pm 0.028$ \\
\hline \multirow[t]{2}{*}{ Diabetes } & 768 & 8 & $\operatorname{Err}_{U}$ & $0.278 \pm 0.011$ & $0.268 \pm 0.008$ \\
\hline & & & Time & $0.025 \pm 0.001$ & $0.355 \pm 0.001$ \\
\hline \multirow[t]{2}{*}{ Image } & 2,310 & 18 & $\operatorname{Err}_{U}$ & $0.070 \pm 0.006$ & $0.063 \pm 0.003$ \\
\hline & & & Time & $2.204 \pm 0.843$ & $120.3 \pm 9.647$ \\
\hline
\end{tabular}

supervised extreme. This is not overly surprising given the poor performance of ridge regression in this example. While the performance improvement of the safe method is much more pronounced in regression, a safety (as defined in Section 4.2 with performance comparable to or better than the pure supervised alternatives) is observed in all examples.

Runtimes were also assessed to compare SCV to CV when $100|L| / n=15 \%$. These results in Table 3.2 further support the claim that our SCV approach performs as well as CV while also providing a computational savings of practical importance.

\subsection{Discussion}

Many semi-supervised learners in the existing literature often require strong smoothness assumptions in order to capitalize on the use of unlabeled data during training. As discussed in the introduction, such assumptions are usually not met in practice, while the unlabeled 
data are likely to have at least some value during training. A computationally efficient safe semi-supervised approach was proposed to address this issue. This approach was specifically designed to adapt to real data by trading-off between the predictions of supervised ridge regression and those of a purely semi-supervised graph-based technique. The approach provided a practical tool which was shown to be competitive on real data in regression and classification settings.

\section{Acknowledgments}

The work of Mark Vere Culp was supported in part by the NSF CAREER/DMS-1255045 grant. The opinions and views expressed in this paper are not reflective of those by the NSF. 


\title{
Chapter 4
}

\section{On Multi-View Learning with Sparse}

\section{Graphs}

\author{
Prithish Banerjee, Michael Morehead, Mark Vere Culp and Kenneth \\ JOSEPH RYAN
}

Submitted in International Conference on Machine Learning

\subsection{Abstract}

Many practical multi-view learning applications involve analyzing data with some degree of noise in the response or the feature data. Existing approaches that rely on view agreement principles or semi-supervised smoothness assumptions are compromised in these circumstances. In this work, an empirical approach is proposed with proven aspects that are ideally suited for such applications by accounting for several key trade-offs encountered in practical multi-view learning problems. The trade-offs include accounting for the bias/variance trade- 
off of prediction error on unlabeled cases, the possibility that the data may be fully viewed or have no appreciable view relationships, and using sparse anchor point methods for detecting manifolds within views. This yields a computationally efficient approach whose effectiveness is demonstrated on both real and simulated data against some established competitors.

\subsection{Introduction}

We develop a semi-supervised regression/classification approach for when feature data come to an analyst in multiple views. The views are a known partitioning of the feature variables and are assumed to naturally follow from the manner in which the data were initially collected. For example, biology (view 1) and chemistry (view 2) data might be used to predict the effectiveness of a drug in a clinical trial setting (Culp and Michailidis, 2009). While the views are known, a given view may have unknown manifolds, and the approach developed herein implicitly finds and uses these manifolds to improve performance only if they have predictive value. Our empirical method is computationally efficient and performs strongly on real data.

Multi-view learning is principled on the concept that the synergy between data generated from multiple sources can be used to improve performance primarily in classification. The survey of Sun (2013) outlines three multi-view learning approaches: (i) combining kernels constructed from each view individually, (ii) view agreement principles, and (iii) subspace learning approaches. Existing multi-view learners based on any of (i)-(iii) tend to work well only if two assumptions hold: sufficiency (i.e., each view is predictive in its own right) and conditional independence (i.e., views are conditionally independent given the response). Relaxing these assumptions has led to many methods including co-training (Wang and Zhou, 2010), manifold co-regularization (Sindhwani and Rosenberg, 2008), model based (Culp, 2011c), and multiple-kernel learning (Bach, 2008; Subrahmanya and Shin, 2010). 
Much work in semi-supervised learning involves semi-supervised smoothness $\left(\mathrm{s}^{2}\right.$-smoothness) assumptions (Lafferty and Wasserman, 2008). The main idea is to relate the conditional density of $y \mid x$ to the marginal density of $x$ to justify the use of unlabeled $x$ 's, so fitting classification borders in the gaps between manifolds in the $x$-space is desirable. Many semisupervised techniques (Chapelle et al., 2006c) can fit the data in this manner including graph cutting (Wang et al., 2013), graph regularization (Belkin et al., 2006; Culp and Ryan, 2013), and S3VM methods (Chapelle et al., 2008). While such a gap-based learner can reduce bias if an $\mathrm{s}^{2}$-smoothness assumption holds, it tends to increase variance by increasing the wiggle-ness of the classification border relative to a supervised approach. Practical issues are known to degrade the performance of many semi-supervised techniques, especially in high-dimensional learning problems where noise blurs the separation of manifolds or is in the response itself (Singh et al., 2009; Culp and Ryan, 2013).

The semi-supervised shrinking ( $\mathrm{s}^{2}$-shrinking) assumption proposed in this work suggests that the unlabeled data could instead be used for variance reduction (as opposed to the bias reduction mentioned in the previous paragraph). The unlabeled data help to identify the view-level manifold structure using proximity graphs constructed by view. They also help regulate the relative degree of the shrinking of unlabeled predictions to decrease prediction variance. For example, after standardizing the labeled responses it is sensible to shrink the predictions of unlabeled extrapolations in the far reaching corners of a manifold, especially in learning problems with noisy data.

In this regard, consider the one-dimensional regression examples with three manifolds in Figure 4.1. Each manifold has a labeled case 'o' and a number of unlabeled cases '"'. The $\mathrm{s}^{2}$-smoothness fit (gray) is a step-function. The proposed $\mathrm{s}^{2}$-shrinking fit (black) is piecewise linear and shrinks predicted values on each manifold more (less) when the unlabeled $x$ is on the opposite (same) side of the manifold as the labeled $x$. (The steepness of the slope is controlled by a tuning parameter in our formulation.) The sign of the slope depends on 

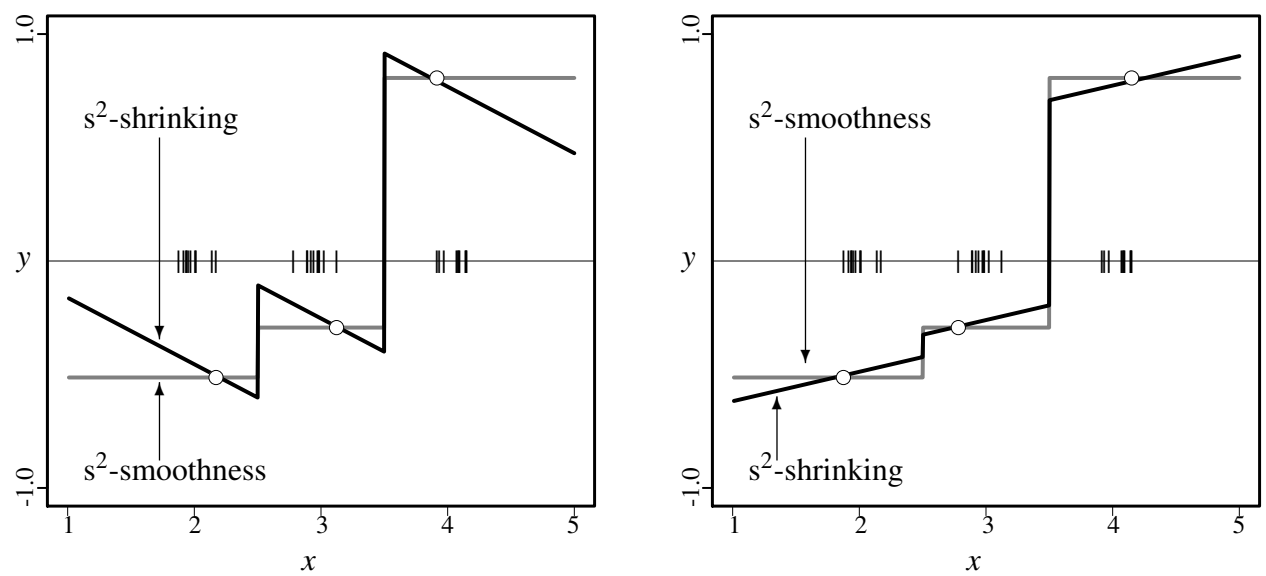

Figure 4.1: Semi-Supervised Shrinking Examples.

the relative position (left or right) of the labeled case within a manifold and the sign of the response. Notice how the sign of the slope changes across the examples in Figure 4.1 when the labeled cases are placed on the opposite side of each manifold, while the step-function is unaffected by this reorientation. In Section $4.3, \mathrm{~s}^{2}$-shrinking is proven to out-perform pure $\mathrm{s}^{2}$-smoothness for noisy learning problems.

Our main contribution is to extend the logic of $\mathrm{s}^{2}$-shrinking into the multi-view learning setting. The proposed optimization in Section 4.4 accounts for practical multi-view tradeoffs including bias versus variance of unlabeled data predictions, noisy data situations, the possibility of misspecification of views via a no-view-distinction term, and adjustments for the relative importance of views. Solving this optimization directly is computationally inefficient even for modest $n$, so the anchor graph methods of Liu et al. (2010), used primarily in large graph construction and hashing problems (Liu et al., 2014), are adjusted to extend the computationally feasibility of our framework in Section 4.5 to a computationally-practical, linear-time version of our technique. This practical technique is shown to perform strongly on real and simulated data in Section 4.6. Concluding remarks are in Section 4.7, and proofs of the main Theorems are in the Appendix. 


\subsection{Semi-Supervised Shrinking}

This section culminates in the presentation of Theorem 2, a single-view result demonstrating the advantages of $\mathrm{s}^{2}$-shrinking over $\mathrm{s}^{2}$-smoothness. Extensions to multiple-views are in Section 4.4 and to sparse, anchor graphs are in Section 4.5 .

Responses $y_{i} \in \mathbb{R}$ for $i=1, \ldots, n$ are assumed to be missing completely at random. Without loss of generality, the first $m$ (first $n-m$ ) observations are the labeled set $L$ (unlabeled set $U$ ). For now, let the $n \times p$ feature matrix $\boldsymbol{X}$ be for a single-view and $\boldsymbol{W}$ be the adjacency matrix for the proximity graph constructed from $X$. The response, feature data, and graph partition as

$$
\left(\begin{array}{c}
\boldsymbol{Y}_{L} \\
\boldsymbol{Y}_{U}
\end{array}\right),\left(\begin{array}{c}
\boldsymbol{X}_{L} \\
\boldsymbol{X}_{U}
\end{array}\right), \text { and }\left(\begin{array}{c}
\boldsymbol{W}_{L L} \boldsymbol{W}_{L U} \\
\boldsymbol{W}_{U L} \boldsymbol{W}_{U U}
\end{array}\right)
$$

with standardized responses $\boldsymbol{Y}_{L}$ and an arbitrary $\boldsymbol{Y}_{U}$.

Regularized joint training

$$
\min _{\boldsymbol{Y}_{U}, \boldsymbol{f}}\left\{\sum_{i \in L} \mathscr{L}\left(\boldsymbol{y}_{i}, \boldsymbol{f}_{i}\right)+\left\|\boldsymbol{Y}_{U}-\boldsymbol{f}_{U}\right\|_{2}^{2}+\lambda \boldsymbol{f}^{T} \boldsymbol{\Delta} \boldsymbol{f}+\gamma\left\|\boldsymbol{Y}_{U}\right\|_{2}^{2}\right\}
$$

with loss function $\mathscr{L}$, combinatorial Laplacian $\Delta$ of $W$ with a partition like $\boldsymbol{W}$ in $(4.1), \lambda>0$, and $\gamma \geq 0$ produces estimators $\left(\widehat{\boldsymbol{f}}, \widehat{\boldsymbol{Y}}_{U}\right)$. An $\widehat{\boldsymbol{f}}$ solving 4.2 with $\gamma>0$ shrinks extrapolations within manifolds in the $\mathrm{s}^{2}$-shrinking manner described in Section 4.2. When $\gamma=0$,

$$
\left(\begin{array}{l}
\widehat{\boldsymbol{f}}_{0 L} \\
\widehat{\boldsymbol{f}}_{0 U}
\end{array}\right) \equiv \arg \min _{\boldsymbol{f}} \sum_{i \in L} \mathscr{L}\left(\boldsymbol{y}_{i}, \boldsymbol{f}_{i}\right)+\lambda f^{T} \boldsymbol{\Delta} f
$$

is an $\widehat{\boldsymbol{f}}$ solving 4.2 since $\boldsymbol{Y}_{U}=f_{U}$ in this case. It is well-understood that optimizations of form (4.3) yield estimators that pass between gaps in manifolds which exhibit empirical 
traits of $\mathrm{s}^{2}$-smoothness (Chapelle et al. 2006c, Culp and Ryan, 2013).

Lemma 11 provides deeper insight into regularized joint training (4.2) and is required for Theorem 2. Define the $m \times m$ matrix $\boldsymbol{\Delta}_{L L}^{\star}$ with $p_{\gamma}=\gamma /(1+\gamma)$ as $\boldsymbol{\Delta}_{L L}^{\star}=\boldsymbol{\Delta}_{L L}-$ $\lambda \Delta_{L U}\left(\lambda \Delta_{U U}+p_{\gamma} \boldsymbol{I}\right)^{-} \Delta_{U L}$. The generalized inverse is only necessary when $\gamma=0$ and $\boldsymbol{\Delta}_{U U}$ is singular even though $\boldsymbol{\Delta}_{L L}^{\star}$ is unique $\forall \gamma \geq 0$.

Lemma 1. If $\left(\widehat{\boldsymbol{f}}, \widehat{\boldsymbol{Y}}_{U}\right)$ solves 4.2$)$, then

$$
\widehat{\boldsymbol{f}}_{L}=\arg \min _{\boldsymbol{f}_{L}} \sum_{i \in L} \mathscr{L}\left(\boldsymbol{y}_{i}, \boldsymbol{f}_{i}\right)+\lambda \boldsymbol{f}_{L}^{T} \boldsymbol{\Delta}_{L L}^{\star} \boldsymbol{f}_{L}
$$

if and only if $\widehat{\boldsymbol{Y}}_{U}=\left(1-p_{\gamma}\right) \widehat{\boldsymbol{f}}_{U}$ and $\widehat{\boldsymbol{f}}_{U}$ satisfies

$$
\left(\lambda \boldsymbol{\Delta}_{U U}+p_{\gamma} \boldsymbol{I}\right) \widehat{\boldsymbol{f}}_{U}=-\lambda \boldsymbol{\Delta}_{U L} \widehat{\boldsymbol{f}}_{L}
$$

Proof. The $\boldsymbol{Y}_{U}$-score of objective 4.2 is $\boldsymbol{Y}_{U}-\boldsymbol{f}_{U}+\gamma \boldsymbol{Y}_{U}=\overrightarrow{0}$ and verifies $\boldsymbol{Y}_{U}=\left(1-p_{\gamma}\right) \boldsymbol{f}_{U}$ as optimal. Vector $\boldsymbol{Y}_{U}$ can then be profiled out of (4.2) because

$$
\gamma\left\|\boldsymbol{Y}_{U}\right\|_{2}^{2}+\left\|\boldsymbol{f}_{U}-\boldsymbol{Y}_{U}\right\|_{2}^{2}=p_{\gamma}\left\|\boldsymbol{f}_{U}\right\|_{2}^{2}
$$

The $f_{U}$-score is thus $\lambda \Delta_{U L} f_{L}+\lambda \Delta_{U U} f_{U}+p_{\gamma} f_{U}=\overrightarrow{0}$ and profiling out $f_{U}$ gives

$$
\begin{aligned}
\lambda \boldsymbol{f}^{T} \boldsymbol{\Delta} \boldsymbol{f}+p_{\gamma} \boldsymbol{f}_{U}^{T} \boldsymbol{f}_{U} & =\lambda \boldsymbol{f}_{L}^{T} \boldsymbol{\Delta}_{L L} \boldsymbol{f}_{L}+2 \lambda \boldsymbol{f}_{L}^{T} \Delta_{L U} \boldsymbol{f}_{U}+\boldsymbol{f}_{U}^{T}\left(\lambda \boldsymbol{\Delta}_{U U}+p_{\gamma} \boldsymbol{I}\right) \boldsymbol{f}_{U} \\
& =\lambda \boldsymbol{f}_{L}^{T} \boldsymbol{\Delta}_{L L} \boldsymbol{f}_{L}+\lambda \boldsymbol{f}_{L}^{T} \boldsymbol{\Delta}_{L U}\left(\lambda \boldsymbol{\Delta}_{U U}+p_{\gamma} \boldsymbol{I}\right)^{-} \boldsymbol{\Delta}_{U L} f_{L} \\
& =\lambda \boldsymbol{f}_{L}^{T} \boldsymbol{\Delta}_{L L}^{\star} \boldsymbol{f}_{L} .
\end{aligned}
$$

By Lemma 1, solving (4.2) $\forall \mathscr{L}$ reduces to a three-step approach: (i) solve (4.4) to get 
$\widehat{\boldsymbol{f}}_{L}$, (ii) use $\widehat{\boldsymbol{f}}_{L}$ in 4.5 to get $\widehat{\boldsymbol{f}}_{U}$, and (iii) set $\widehat{\boldsymbol{Y}}_{U}$ using $\widehat{\boldsymbol{f}}_{U}$.

We seek a single-view learning bound to identify circumstances when $\mathrm{s}^{2}$-shrinking $(\gamma>$ 0 ) out-performs s $\mathrm{s}^{2}$-smoothness $(\gamma=0)$. With this in mind, let $\left\{d_{i}, \boldsymbol{o}_{i}\right\}$ be an eigen-decomposition of $\lambda \Delta_{U U}$ where $d_{1} \geq \cdots \geq d_{n-m}>0$ (because a bound turns out to be trivial if $d_{n-m}=$ 0 ). For any $\gamma \geq 0$, project vector $-\lambda \Delta_{U L} \widehat{f}_{L}=\sum_{i=1}^{n-m} \widehat{a}_{\gamma i} \boldsymbol{o}_{i}$ and consider Assumptions 2.1 and 2.2 .

Assumption 2.1: $\forall \varepsilon>0 \exists \widetilde{\gamma}>0$ such that $\max _{i} \frac{\left|\widehat{a}_{0 i}-\widehat{a}_{\gamma_{i}}\right|}{d_{i}} \leq \frac{\varepsilon}{\sqrt{n-m}} \forall \gamma<\widetilde{\gamma}$.

Assumption 2.2: $\forall \widehat{\boldsymbol{f}}_{L}$ solving 4.4$], \quad a_{i}=-\boldsymbol{o}_{i}^{T} \mathbb{E}\left[\lambda \boldsymbol{\Delta}_{U L} \widehat{\boldsymbol{f}}_{0 L}\right]$ and $\left.\operatorname{Var}\left[\boldsymbol{o}_{i}^{T} \lambda \boldsymbol{\Delta}_{U L} \widehat{\boldsymbol{f}}_{0 L}\right]=\boldsymbol{o}_{i}^{T} \boldsymbol{\Sigma} \boldsymbol{o}_{i} \sigma^{2}<\infty .1\right]$

Under squared error loss, 4.4 yields the labeled estimator $\widehat{\boldsymbol{f}}_{L}=\left(\boldsymbol{I}+\lambda \boldsymbol{\Delta}_{L L}^{\star}\right)^{-1} \boldsymbol{Y}_{L}$ satisfying Assumption 2.1. This $\widehat{\boldsymbol{f}}_{L}$ also satisfies Assumption 2.2 if $\operatorname{Var}\left[\boldsymbol{Y}_{L}\right]=\sigma^{2} \boldsymbol{I}$.

Theorem 2. Let Assumptions 2.1 and 2.2 hold, $\boldsymbol{m}_{U}=\sum_{i=1}^{n-m} b_{i} \boldsymbol{o}_{i}$ be the vector of expected responses given $\boldsymbol{X}_{U}$, and $M=\sup _{\gamma} \sum_{i=1}^{n-m} \mathbb{E}\left[\left|\frac{\widehat{a}_{0 i}}{d_{i}+p_{\gamma}}-b_{i}\right|\right]$. If

$$
\sigma^{2}>\left(\frac{\sum_{i=1}^{n-m}\left(a_{i} b_{i} d_{i}-a_{i}^{2}\right) / d_{i}^{3}}{\sum_{i=1}^{n}\left\|\mathbf{\Sigma}^{1 / 2} \boldsymbol{o}_{i}\right\|_{2}^{2} / d_{i}^{3}}\right)_{+},
$$

then $\mathbb{E}\left[\left\|\widehat{\boldsymbol{f}}_{U}-\boldsymbol{m}_{U}\right\|_{2}^{2}\right]<\mathbb{E}\left[\left\|\widehat{\boldsymbol{f}}_{0 U}-\boldsymbol{m}_{U}\right\|_{2}^{2}\right]+\mathscr{O}\left(\frac{2 M}{\sqrt{n-m}} \varepsilon+\varepsilon^{2}\right)$.

The terms in performance bound (4.8) are positive if $b_{i} d_{i}-a_{i}>0$ or $\boldsymbol{o}_{i}^{T} \boldsymbol{m}_{U}>-\boldsymbol{o}_{i}^{T} \boldsymbol{\Delta}_{U U}^{-1}$ $\Delta_{U L} \mathbb{E}\left[\widehat{\boldsymbol{f}}_{0 L}\right]$, i.e., $\mathrm{s}^{2}$-shrinking essentially works unless $\boldsymbol{o}_{i}^{T} \boldsymbol{m}_{U}$ is much larger than expected under $\mathrm{s}^{2}$-smoothness for larger $i$ corresponding to the smaller eigenvalues $d_{i}$. This is intuitive since it is difficult to predict large $\boldsymbol{m}_{U}$ and nothing concrete about performance can be assessed for either assumption in this case. By Theorem 2 , larger $\sigma^{2}$ only further favors $\mathrm{s}^{2}$-shrinking.

\footnotetext{
${ }^{1}$ All expectations are conditional given $\boldsymbol{W}$ and fixed $\lambda$.
} 
Table 4.1: Square Matrix $\boldsymbol{A}^{(\ell)}, \boldsymbol{A}$ and Vector $\boldsymbol{a}^{(\ell)}, \boldsymbol{a}$ Notation.

\begin{tabular}{|c|c|}
\hline Notation & Definition \\
\hline $\boldsymbol{A}^{(1)} \otimes \boldsymbol{A}^{(2)}$ & The Kronecker product. \\
\hline $\boldsymbol{A} \succeq 0$ & $\boldsymbol{A}$ is positive semi-definite. \\
\hline $\operatorname{vec}_{\ell \leq q}\left(\boldsymbol{a}^{(\ell)}\right)$ & Vector from stacking $\left\{\boldsymbol{a}^{(\ell)}\right\}_{\ell=1}^{q}$. \\
\hline $\operatorname{vec}_{\ell \leq q}\left(\boldsymbol{a}, \boldsymbol{a}^{(\ell)}\right)$ & Now stack $\boldsymbol{a}$ on top of vec $\operatorname{vec}_{\ell \leq q}\left(\boldsymbol{a}^{(\ell)}\right)$. \\
\hline $\operatorname{diag}_{\ell \leq q}\left(\boldsymbol{A}^{(\ell)}\right)$ & Block diagonal matrix $\left\{\boldsymbol{A}^{(\ell)}\right\}_{\ell=1}^{q}$. \\
\hline $\operatorname{diag}_{\ell \leq q}\left(\boldsymbol{A}, \boldsymbol{A}^{(\ell)}\right)$ & Now the blocks are $\boldsymbol{A}, \operatorname{diag}_{\ell \leq q}\left(\boldsymbol{A}^{(\ell)}\right)$ \\
\hline $\operatorname{diag}(\boldsymbol{a})$ & 1 matrix w \\
\hline
\end{tabular}

\subsection{Joint Multi-View Learning Framework}

A multi-view model is presented. Formally, assume that the $n \times p$ matrix $\boldsymbol{X}$ is partitioned column-wise into $q$ views $\left\{\boldsymbol{X}^{(\ell)}\right\}_{\ell=1}^{q}$ where each $\boldsymbol{X}^{(\ell)}$ is an $n \times p_{\ell}$ matrix. The $\ell$ th adjacency graph matrix $\boldsymbol{W}^{(\ell)}$ and its Laplacian $\boldsymbol{\Delta}^{(\ell)}$ constructed from $\boldsymbol{X}^{(\ell)}$ each emit partitions (4.1). Other important notational conventions are listed in Table 4.1

Our contention is that the expected response for extrapolations within manifolds may not fully conform to a manifold or $\mathrm{s}^{2}$-smoothness assumption, so the variance of unlabeled predictions can be improved by shrinking without necessarily incurring additional squared bias. This rationale helps to motivate the optimization problem

$$
\begin{aligned}
\min _{\substack{\boldsymbol{Y}_{U}, \alpha, \boldsymbol{\beta}, f^{(1)}, \ldots, f^{(q)}}}\left\{\sum_{i \in L} \mathscr{L}\left(\boldsymbol{y}_{i}, \boldsymbol{\eta}_{i}\right)\right. & +\left\|\boldsymbol{Y}_{U}-\boldsymbol{\eta}_{U}\right\|_{2}^{2}+\lambda_{0}\|\boldsymbol{\beta}\|_{2}^{2} \\
& \left.+\sum_{\ell=1}^{q} \lambda_{\ell} \boldsymbol{f}^{(\ell)^{T}} \boldsymbol{\Delta}^{(\ell)} \boldsymbol{f}^{(\ell)}+\gamma\left\|\boldsymbol{Y}_{U}\right\|_{2}^{2}\right\},
\end{aligned}
$$

where $\boldsymbol{\eta}=\alpha \overrightarrow{1}+\boldsymbol{X} \boldsymbol{\beta}+\sum_{\ell=1}^{q} \boldsymbol{f}^{(\ell)}$

$\mathscr{L}$ is a loss function, $\gamma \geq 0$, and $\lambda_{\ell} \geq 0$ for $\ell=0, \ldots, q$. 
Predictions (4.10) take into account many trade-offs that arise in practical multi-view problems. First, assume $\boldsymbol{\beta}=\overrightarrow{0}\left(\lambda_{0}=\infty\right), \gamma=0$, and $q=1$. In this case, 4.9 is the labeled loss problem (4.3) from Section 4.3. The $\gamma$ parameter regulates prediction variability on extrapolation extremes of a manifold as described previously. For $q>1$, the $\lambda_{\ell}$ act to tradeoff the importance of each view relative to the overall function estimate. The $\boldsymbol{\beta}$ term accounts for the possibility that either there are no actual manifolds in the data or the manifolds are not practically useful for prediction. In either case, regularization can compromise towards an overall no-view-distinction estimator, $\alpha \overrightarrow{1}+\boldsymbol{X} \boldsymbol{\beta}$. Accounting for all of these trade-offs on real data by appropriately constructing the $q$ graphs and estimating the $q+2$ tuning parameters is a key practical challenge with fitting $\widehat{\boldsymbol{\eta}}$.

Proposition 5 extends Lemma 1 to the multi-view setting, provides insight into the computational burden, and motivates its sparse version to come in Section 4.5 .

Proposition 5. There exists $a(1+p+m q) \times(1+p+m q)$ matrix $\boldsymbol{P} \succeq 0$ s.t. $\left(\widehat{\alpha}, \widehat{\beta}, \widehat{\boldsymbol{f}}_{L}^{(1)}, \ldots, \widehat{\boldsymbol{f}}_{L}^{(q)}\right)$ solves

$$
\min _{\substack{\alpha, \boldsymbol{\beta}, \boldsymbol{f}_{L}^{(1)}, \ldots, \boldsymbol{f}_{L}^{(q)}}} \sum_{i \in L} \mathscr{L}\left(\boldsymbol{y}_{i}, \boldsymbol{\eta}_{i}\right)+\left\|\boldsymbol{P}^{\frac{1}{2}} \underset{\ell \leq q}{\ell \leq q}\left(\left(\begin{array}{l}
\alpha \\
\beta
\end{array}\right), \boldsymbol{f}_{L}^{(\ell)}\right)\right\|_{2}^{2}
$$

if and only if $\left(\widehat{\alpha}, \widehat{\boldsymbol{\beta}}, \widehat{\boldsymbol{Y}}_{U}, \widehat{\boldsymbol{f}}^{(1)}, \ldots, \widehat{\boldsymbol{f}}^{(q)}\right)$ solves 4.9p for any $\left(\widehat{\boldsymbol{Y}}_{U}, \widehat{\boldsymbol{f}}_{U}^{(1)}, \ldots, \widehat{\boldsymbol{f}}_{U}^{(q)}\right)$ satisfying $\widehat{\boldsymbol{Y}}_{U}=\left(1-p_{\gamma}\right) \widehat{\boldsymbol{\eta}}_{U}$ and

$$
\left(\operatorname{diag}_{\ell \leq q}\left(\lambda_{\ell} \boldsymbol{\Delta}_{U U}^{(\ell)}\right)+p_{\gamma} \boldsymbol{J} \otimes \boldsymbol{I}\right) \underset{\ell \leq q}{\operatorname{vec}}\left(\widehat{\boldsymbol{f}}_{U}^{(\ell)}\right)=-\operatorname{vec}_{\ell \leq q}\left(\lambda_{\ell} \boldsymbol{\Delta}_{U L}^{(\ell)} \widehat{\boldsymbol{f}}_{L}^{(\ell)}+\alpha \overrightarrow{1}+\boldsymbol{X}_{U} \boldsymbol{\beta}\right),
$$

where $\mathbf{J}$ is a $q \times$ q matrix of 1's.

Proof. The proof concept is based on the observation that the sum of the four penalty terms in (4.9) not involving loss function $\mathscr{L}$ equals a quadratic form with the vector of $n-m+$ 
$1+p+n q$ unknown variables and a known, symmetric, positive semi-definite matrix. When this observation is combined with the use of labeled loss, optimal $\boldsymbol{Y}_{U}$ and the $\boldsymbol{f}_{U}^{(\ell)}$ must be linear in $\alpha, \boldsymbol{\beta}$, and the $f_{L}^{(\ell)}$, so the existence of matrix $\boldsymbol{P}$ follows.

To see this, proceed as in the proof of Lemma 1 to get $\boldsymbol{Y}_{U}=\left(1-p_{\gamma}\right) \eta_{U}$ analogous to 4.6 and profile out $\boldsymbol{Y}_{U}$. The score for any $\boldsymbol{f}_{U}^{(\ell)}$ reduces to $\lambda_{\ell} \boldsymbol{\Delta}_{U L}^{(\ell)} \boldsymbol{f}_{L}^{(\ell)}+\lambda_{\ell} \boldsymbol{\Delta}_{U U}^{(\ell)} \boldsymbol{f}_{U}^{(\ell)}+p_{\gamma} \boldsymbol{\eta}_{U}=$ $\overrightarrow{0}$, verifies 4.12), and shows that each $\boldsymbol{f}_{U}^{(\ell)}$ is linear in $\alpha, \boldsymbol{\beta}$, and $\left\{\boldsymbol{f}_{L}^{(\ell)}\right\}_{\ell=1}^{q}$. In addition, reduction (4.7) generalizes to

$$
\begin{gathered}
\left\|\left(\operatorname{diag}_{\ell \leq q}\left(\lambda_{\ell} \Delta_{L L}^{(\ell)}\right)\right)^{\frac{1}{2}} \underset{\ell \leq q}{\operatorname{vec}}\left(f_{L}^{(\ell)}\right)\right\|_{2}^{2}- \\
\left\|\left(\operatorname{diag}_{\ell \leq q}\left(\lambda_{\ell} \Delta_{U U}^{(\ell)}\right)+p_{\gamma} \boldsymbol{J} \otimes \boldsymbol{I}\right)^{-\frac{1}{2}} \underset{\ell \leq q}{\operatorname{vec}}\left(\lambda_{\ell} \boldsymbol{\Delta}_{U L}^{(\ell)} \boldsymbol{f}_{L}^{(\ell)}\right)\right\|_{2}^{2},
\end{gathered}
$$

which equals the penalty due to the lower-right $q m \times q m$ block of $\boldsymbol{P}$.

The matrix inverses and multiplications necessary for computing the unlabeled estimates (4.12) is accounted for by computing $\boldsymbol{P}$ from Proposition 5 . If $\boldsymbol{\xi}(m, q, p)$ is the order of the computational burden for fitting 4.11 given $\boldsymbol{P}$, then $\xi(m, q, p)=(m q+p)^{3}$ under squared error loss. Putting this together, the overall computational complexity for the full multi-view fit $\widehat{\boldsymbol{\eta}}$ in 4.10 is of order $\xi(m, q, p)+(q(n-m))^{3}+(n-m) p^{2}$ after $q$ quadratic in $n$ graph construction phases.

Remark 2. Proposition 5 provides a reduction that can produce a unique solution $\widehat{\boldsymbol{\eta}}$ depending on the loss function $\mathscr{L}$. Functional uniqueness of the terms $\widehat{\boldsymbol{f}}^{(\ell)}$ in $\widehat{\boldsymbol{\eta}}$ is non-trivial due to non-zero vectors in the intersection of the null spaces of the $\boldsymbol{\Delta}^{(\ell)}$ Culp and Ryan. 2013), but is possible with modified back-fitting algorithms (Buja et al. 1989). 


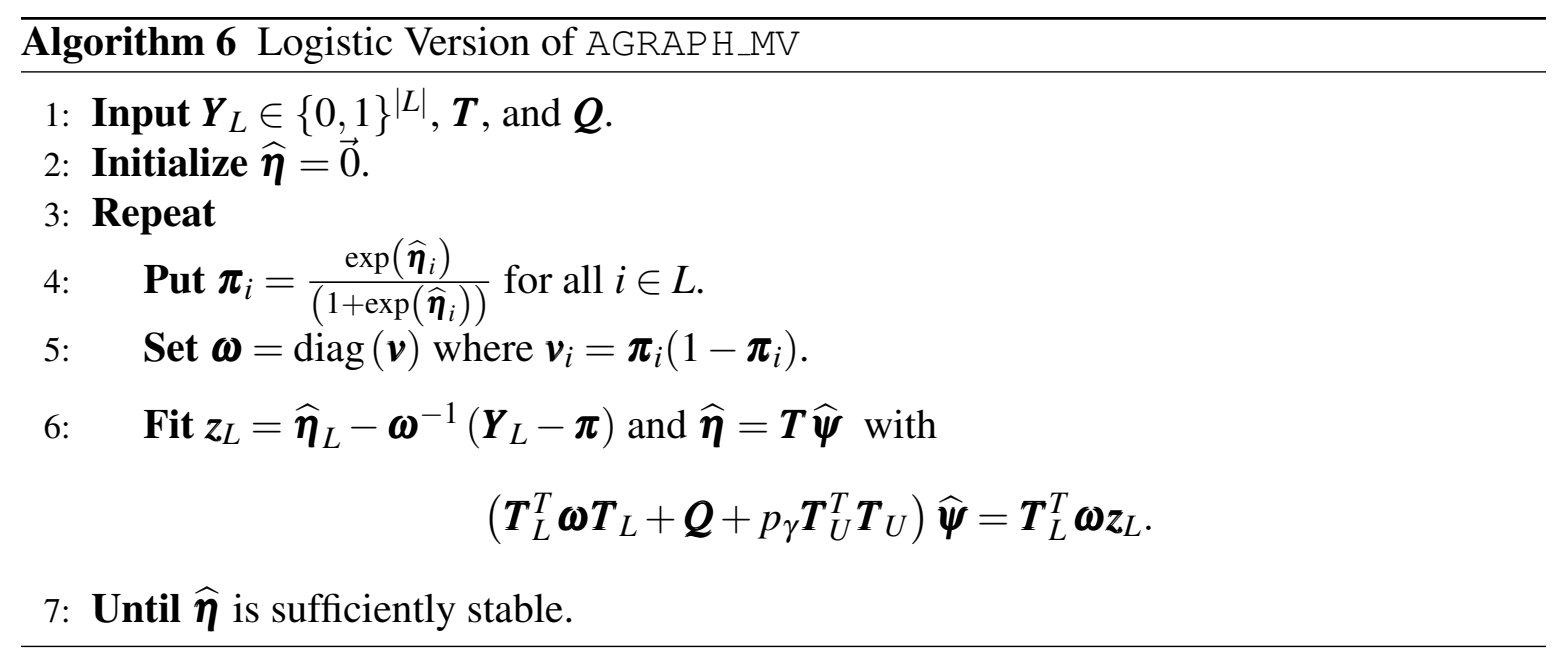

\subsection{Joint Training with Sparse Graphs}

The goal in this section is to adapt the anchor graph approach of Liu et al. (2010) to solve the joint multi-view learning criterion (4.9). The general Anchor graph discussion from Section 2.3 and the LAE algorithm specifically from Section 2.3.2 are used to generate a matrix $\boldsymbol{Z}^{(\ell)}$ for each view $i$ individually with $k_{\ell}$ (similar as $m$ in Section 2.3.1). That is, for each view $\ell$ the $n \times p_{\ell}$ feature data $\boldsymbol{X}^{(\ell)}$ is processed to generate reduced matrix $\boldsymbol{Z}^{(\ell)}$ which then in-turn generates the Anchor Graphs $\boldsymbol{W}^{(\ell)}=\boldsymbol{Z}^{(\ell)} \operatorname{diag}\left(\boldsymbol{Z}^{(\ell)^{T}} \overrightarrow{1}\right)^{-1} \boldsymbol{Z}^{(\ell)^{T}}$, corresponding Laplacians $\boldsymbol{\Delta}^{(\ell)}=\boldsymbol{I}-\boldsymbol{W}^{(\ell)}$, and reduced Laplacian $\widetilde{\boldsymbol{\Delta}}^{(\ell)}=\boldsymbol{Z}^{(\ell)^{T}} \boldsymbol{\Delta}^{(\ell)} \boldsymbol{Z}^{(\ell)}$. This process is fully discussed in Section 2.3.

Using anchor graphs in 4.9 with smooth functions $\boldsymbol{f}^{(\ell)}=\boldsymbol{Z}^{(\ell)} \boldsymbol{\beta}^{(\ell)}$ yields a linear fit $\widehat{\boldsymbol{\eta}}=\boldsymbol{X} \widehat{\boldsymbol{\beta}}+\sum_{\ell=1}^{q} \boldsymbol{Z}^{(\ell)} \widehat{\boldsymbol{\beta}}^{(\ell)}$. Let $\widetilde{p}=\sum_{\ell=1}^{q} k_{\ell}+p$. Optimization 4.9 then simplifies to

$$
\begin{aligned}
\min _{\substack{\boldsymbol{Y}_{U}, \boldsymbol{\beta}, \boldsymbol{\beta}^{(1)}, \ldots, \boldsymbol{\beta}^{(q)}}}\left\{\sum_{i \in L} \mathscr{L}\left(\boldsymbol{y}_{i}, \boldsymbol{T}_{i} \boldsymbol{\psi}\right)\right. & +\left\|\boldsymbol{Y}_{U}-\boldsymbol{T}_{U} \boldsymbol{\psi}\right\|_{2}^{2}+\lambda_{0}\|\boldsymbol{\beta}\|_{2}^{2} \\
& \left.+\sum_{\ell=1}^{q} \lambda_{\ell} \boldsymbol{\beta}^{(\ell)^{T}} \widetilde{\boldsymbol{\Delta}}^{(\ell)} \boldsymbol{\beta}^{(\ell)}+\gamma\left\|\boldsymbol{Y}_{U}\right\|_{2}^{2}\right\},
\end{aligned}
$$

where $\boldsymbol{\psi}=\operatorname{vec}_{\ell \leq q}\left(\boldsymbol{\beta}, \boldsymbol{\beta}^{(\ell)}\right)$, 
and $\boldsymbol{T}$ is the $n \times \widetilde{p}$ column-wise concatenation of $\boldsymbol{X}$ and $\left\{\boldsymbol{Z}^{(\ell)}\right\}_{\ell=1}^{q}$. Proposition 6 profiles $\boldsymbol{Y}_{U}$ out of 4.13).

Proposition 6. A solution to 4.13 is given by $\widehat{\boldsymbol{\eta}}=\boldsymbol{T} \widehat{\boldsymbol{\psi}}$, where the $\widetilde{p}$-length vector $\widehat{\boldsymbol{\psi}}$ is a solution to

$$
\begin{gathered}
\min _{\boldsymbol{\psi}} \sum_{i \in L} \mathscr{L}\left(\boldsymbol{y}_{i}, \boldsymbol{T}_{i} \boldsymbol{\psi}\right)+\boldsymbol{\psi}^{T}\left(p_{\gamma} \boldsymbol{T}_{U}^{T} \boldsymbol{T}_{U}+\boldsymbol{Q}\right) \boldsymbol{\psi} \\
\text { with } \boldsymbol{Q}=\operatorname{diag}_{\ell \leq q}\left(\lambda_{0} \boldsymbol{I}, \lambda_{\ell} \widetilde{\boldsymbol{\Delta}}^{(\ell)}\right)
\end{gathered}
$$

Proof. The $\boldsymbol{Y}_{U}$ terms in objective in 4.13 have score $\boldsymbol{Y}_{U}-\boldsymbol{T}_{U} \boldsymbol{\psi}+\gamma \boldsymbol{Y}_{U}=\overrightarrow{0}$ which verifies $\boldsymbol{Y}_{U}=\left(1-p_{\gamma}\right) \boldsymbol{T}_{U} \boldsymbol{\psi}$. Vector $\boldsymbol{Y}_{U}$ is then profiled out of 4.2$]$ through the identity $\gamma\left\|\boldsymbol{Y}_{U}\right\|_{2}^{2}+$ $\left\|\boldsymbol{T}_{U} \boldsymbol{\psi}-\boldsymbol{Y}_{U}\right\|_{2}^{2}=p_{\gamma}\left\|\boldsymbol{T}_{U} \boldsymbol{\psi}\right\|_{2}^{2}$

The computational complexity of solving (4.14) is substantially less than that of solving 4.9. with general $\boldsymbol{W}^{(\ell)}$, although the actual complexity depends on the loss function. A solution to 4.13, referred to as AGRAPH_MV, is directly fit in Section 4.6 under both squared error loss and logistic loss. For squared error loss, $\widehat{\boldsymbol{\psi}}$ is a solution to $\left(\boldsymbol{T}_{L}^{T} \boldsymbol{T}_{L}+p_{\gamma} \boldsymbol{T}_{U}^{T} \boldsymbol{T}_{U}+\boldsymbol{Q}\right) \widehat{\boldsymbol{\psi}}$ $=\boldsymbol{T}_{L}^{T} \boldsymbol{Y}_{L}$, and the computational complexity is of order $\widetilde{p}^{3}+\widetilde{p}^{2} m$ after $q$ linear in $n$ anchor graph construction phases. Logistic loss was solved iteratively using Algorithm 1 and has a similar complexity.

\subsubsection{Multi-View Learning Bound}

A corresponding learning bound is proven for the AGRAPH_MV based on Assumptions 4.1 and 4.2.

Assumption 4.1: Any $\widehat{\boldsymbol{\psi}}_{\gamma}$ solving 4.13 satisfies $\left(\boldsymbol{R}+p_{\gamma} \boldsymbol{T}_{U}^{T} \boldsymbol{T}_{U}\right) \widehat{\boldsymbol{\psi}}_{\gamma}=\boldsymbol{R} \widehat{\boldsymbol{c}}$ for some $\boldsymbol{R} \succeq 0$ 
Assumption 4.2: For each $j \leq \widetilde{p}, \mathbb{E}\left[\boldsymbol{w}_{j}^{T} \widehat{\boldsymbol{c}}\right]=\mu_{j}$ and $\operatorname{Var}\left[\boldsymbol{w}_{j}^{T} \widehat{\boldsymbol{c}}\right]=\boldsymbol{w}_{j}^{T} \widetilde{\boldsymbol{\Sigma}} \boldsymbol{w}_{j} \sigma^{2}<\infty$, where $\widetilde{\boldsymbol{\Sigma}} \succeq 0$ and $\left\{\tau_{j}, \boldsymbol{w}_{j}\right\}$ is a generalized eigen-decomposition satisfying $\boldsymbol{T}_{U}^{T} \boldsymbol{T}_{U} \boldsymbol{w}_{j}=\tau_{j} \boldsymbol{R} \boldsymbol{w}_{j}$ with $\boldsymbol{w}_{j}^{T} \boldsymbol{R} \boldsymbol{w}_{j}=1$.

Assumption 4.1 is satisfied by the squared error loss version of AGRAPH MV with $\boldsymbol{R}=$ $\boldsymbol{T}_{L}^{T} \boldsymbol{T}_{L}+\boldsymbol{Q}$ where $\boldsymbol{Q}$ is from 4.14 ) and with $\widehat{\boldsymbol{c}}$ satisfying $\boldsymbol{R} \widehat{\boldsymbol{c}}=\boldsymbol{T}_{L}^{T} \boldsymbol{Y}_{L}$. In addition, the linear model $\mathbb{E}\left[\boldsymbol{Y}_{L}\right]=\boldsymbol{T}_{L} \boldsymbol{\theta}$ and $\operatorname{Var}\left[\boldsymbol{Y}_{L}\right]=\sigma^{2} \boldsymbol{I}$ implies Assumption 4.2. A similar analysis shows that Assumptions 4.1 and 4.2 hold for the logistic version of AGRAP H_MV if $\mathbb{E}\left[\boldsymbol{z}_{L}\right]=\boldsymbol{T}_{L} \boldsymbol{\theta}$.

In general, $\boldsymbol{\theta}=\sum_{j=1}^{\widetilde{p}} q_{j} \boldsymbol{w}_{j}$ is the coefficient vector for the expected linearized responses $\boldsymbol{z}_{L}$ from (4.14).

Theorem 3. Assume $\boldsymbol{R} \succ 0$ and that Assumptions 4.1 and 4.2 hold. If $\sigma^{2}>\left(\frac{\sum_{j=1}^{\widetilde{p}}\left(\mu_{j} q_{j}-\mu_{j}^{2}\right) \tau_{j}^{2}}{\sum_{j=1}^{\tilde{p}}\left\|\tau_{j} \widetilde{\boldsymbol{\Sigma}}^{1 / 2} \boldsymbol{w}_{j}\right\|_{2}^{2}}\right)$, then $\mathbb{E}\left[\left\|\boldsymbol{T}_{U}\left(\widehat{\boldsymbol{\psi}}_{\gamma}-\boldsymbol{\theta}\right)\right\|_{2}^{2}\right]<\mathbb{E}\left[\left\|\boldsymbol{T}_{U}\left(\widehat{\boldsymbol{\psi}}_{0}-\boldsymbol{\theta}\right)\right\|_{2}^{2}\right]$.

Theorem 3 generalizes the $\mathrm{s}^{2}$-shrinking bound in Theorem 2 to our multi-view, sparsegraph setting 4.13 .

\subsection{Empirical Performance}

The empirical competitiveness of the proposed approach, AGRAPH_MV, is now demonstrated on simulated and real data with $q=2$ views. The AGRAPH MV was fit with $s_{1}=s_{2}=5$ and $k_{1}=k_{2}=k$ with $k=\lceil 0.1 n\rceil$ whenever $n<1500$ and $k=500$ otherwise. Anchor points were determined by $\mathrm{k}$-means, and the LAE algorithm was used to fit the anchor graphs (Liu et al., 2010). A natural robustness to these choices has been demonstrated in prior work (Liu et al. 2014) and is consistent with our experience. All additional tuning parameters were estimated with 3 -fold cross-validation over the grid $\left(\lambda_{0}, \lambda_{1}, \lambda_{2}, \gamma\right) \in$ $\{0.01,0.1,1.0,10.0\}^{3} \times\{0.0,0.001,0.01\}$. In fitting this approach, computational and exact 


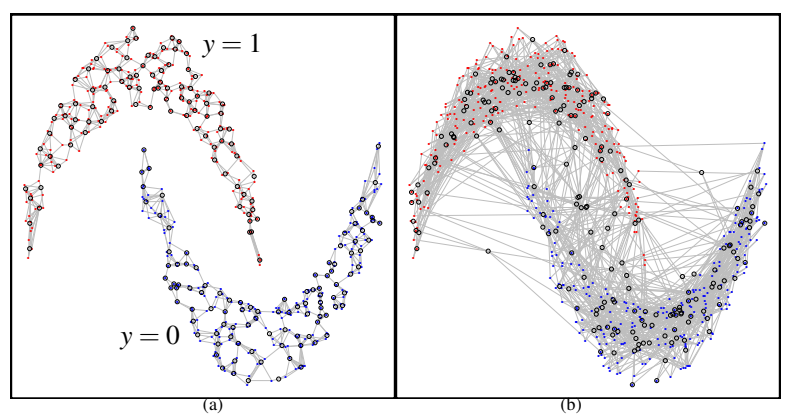

Figure 4.2: Simulated Two Moons Example.

singularities arise in practice and can dramatically slow-down implementation due to a non-

unique $\widehat{\boldsymbol{\psi}}$. This computational issue was addressed by replacing matrix $\boldsymbol{Q}$ from optimization (4.14) with $\boldsymbol{Q}+1 e-5 \times \boldsymbol{I}$.

Three competing techniques were fit in $\mathrm{R}(\mathrm{R}$ Core Team, 2015). Co-FTF is an easy-toimplement variation of co-training using generic supervised learners (Culp and Michailidis 2009). The SVM with RBF was used as the generic learner for each view with the kernlab package (Karatzoglou et al., 2004). Manifold co-regularization with RBF was also fit where the tuning parameters were all optimized as described in Sindhwani and Rosenberg (2008). Last, the semi-supervised AnchorGraphReg was fit with graph parameters $s=5$ and $k=\lceil 0.1 n\rceil$ whenever $n<1500$ and $k=500$ otherwise and with cross-validation for the regularization parameter (Liu et al., 2010).

\subsubsection{Simulation}

As noted in Section 4.2, the effectiveness of a view agreement approach depends on the assumption that each view is sufficiently predictive on its own (Sun, 2013). Here, simulated data sets with $n=500$ observations demonstrate issues with this type of sufficiency assumption in the presence of noise. The first view is the two moons plotted in Figure 4.2 (a) that determine the binary response. The small plotted points are observations while the large circles are anchor points. The graph connections are those linking observations with only the 
Table 4.2: Unlabeled Error Rates: Two Moons and Noise View $p_{2}$.

\begin{tabular}{|r|c|c|c|}
\hline Technique & $|L| / n$ & $p_{2}=2$ & $p_{2}=5$ \\
\hline AGRAPH_MV & $15 \%$ & $0.01 \pm 0.00$ & $0.01 \pm 0.00$ \\
& $30 \%$ & $0.01 \pm 0.00$ & $0.01 \pm 0.00$ \\
\hline Co-FTF (SVM) & $15 \%$ & $0.05 \pm 0.01$ & $0.06 \pm 0.01$ \\
& $30 \%$ & $0.03 \pm 0.01$ & $0.04 \pm 0.01$ \\
\hline Man_Co & $15 \%$ & $0.13 \pm 0.02$ & $0.15 \pm 0.02$ \\
& $30 \%$ & $0.09 \pm 0.00$ & $0.11 \pm 0.01$ \\
\hline Anchor- & $15 \%$ & $0.03 \pm 0.00$ & $0.06 \pm 0.00$ \\
GraphReg & $30 \%$ & $0.02 \pm 0.00$ & $0.05 \pm 0.00$ \\
\hline
\end{tabular}

first $p_{1}=2$ columns of $\boldsymbol{X}$. The second view is $p_{2}=2$ (simulation 1$)$ or $p_{2}=5$ (simulation 2 ) columns of standardized random normal noise. Each simulation was run with $15 \%$ and $30 \%$ labeled cases, and the unlabeled classification error rates were recored. This was repeated 50 times to produce the mean unlabeled error rate \pm margin of error entries in Table 4.2 .

The presence of noise corrupts all techniques (i.e., no approach perfectly identifies the moons). The proposed AGRAPH_MV mitigates the effect of noise by decreasing $\lambda_{1}$ (increasing $\lambda_{2}$ ) and is most successful. The Co-FTF (SVM) compromises with the noisy view in an attempt to improve performance that backfires. For Man_Co, the view agreement term $\left\|\boldsymbol{f}_{1 U}-\boldsymbol{f}_{2 U}\right\|_{2}^{2}$ misleads the approach as it also compromises an informative view with a noise view. For the AnchorGraphReg, noise corrupts the ability to identify edges appropriately as shown in Figure 4.2 (b), i.e., the graph connections are those using all $p_{1}+p_{2}$ columns of $\boldsymbol{X}$ to generate the anchor graph. Softer variations of this albeit extreme example are likely occurring in the real data examples of Section 4.6.2 and helps explain the performance improvement due to AGRAPH_MV.

\subsubsection{Real Data}

Real data tests were performed on the four, challenging, multi-view examples listed in Table 4.3. For the neuroscience (brain) data set, entry statistics were mined from NeuroMor- 

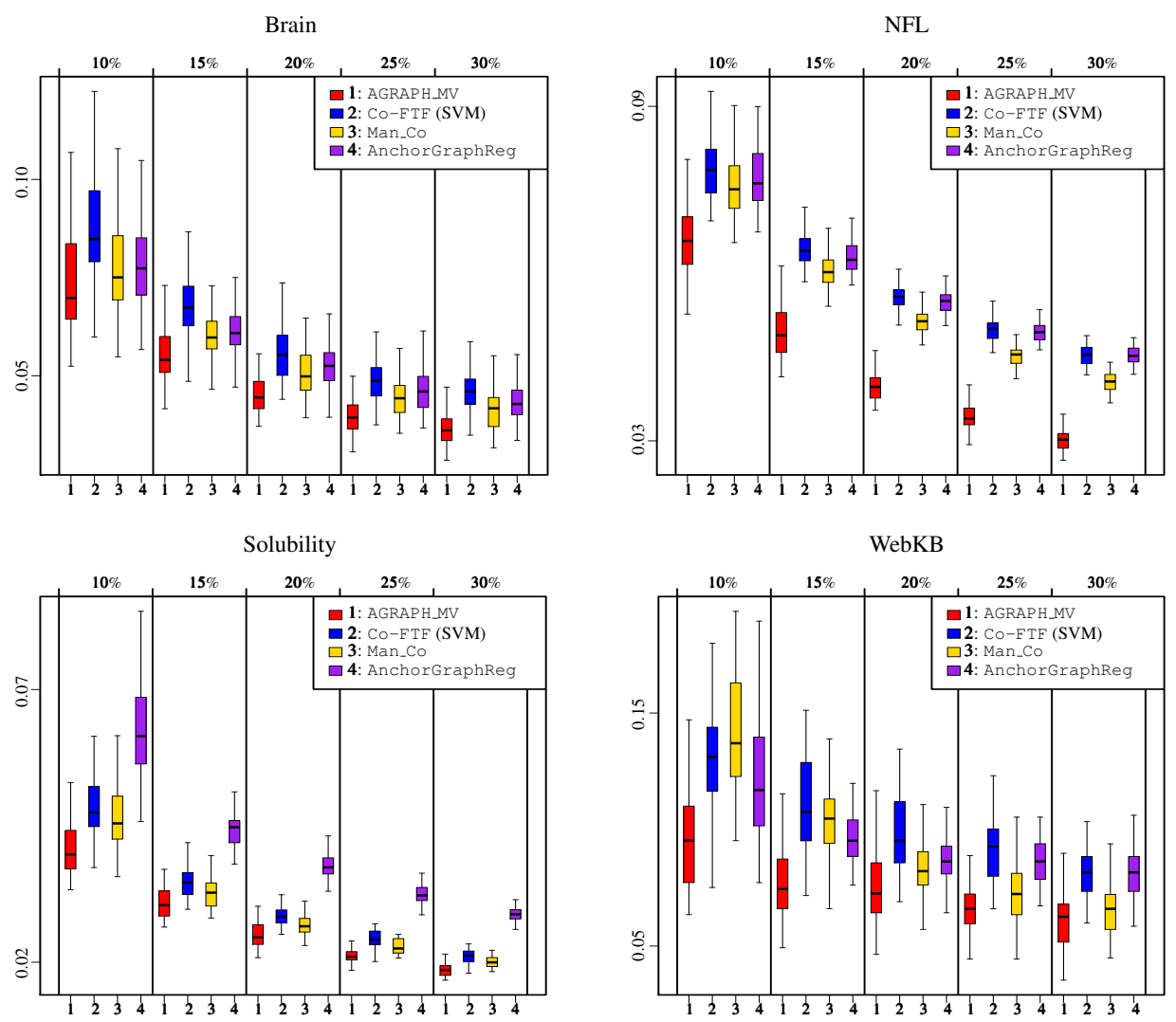

Figure 4.3: Unlabeled Performance on Real Data Sets.

pho.Org with the two views obtained as either the extended L-Measure or distribution statistics mined via the TREES toolbox (Ascoli et al., 2007; Cuntz et al., 2010) with the intent of predicting median age. Certain measurements such as species were not part of either view, but were used in $X$ for the proposed method. The NFL data, which was mined from profootball-reference.com, consists of offensive and defensive season summary statistics for all NFL games from 2000 through the 2015 playoffs with the intent of predicting the observed point spread (home minus away). The solubility data consists of compounds that are either described by chemical fingerprints (e.g., the presence or absence of chemical structures) or chemical properties (e.g., molecular weight), and the goal is to predict compound solubility (Kuhn and Johnson, 2013). The final data set was the classification problem of predicting whether or not a university website was a course page using the link and text views from the 
Table 4.3: Multi-View Data Sets.

\begin{tabular}{|l|l|l|}
\hline Data Set $\left(n, p, p_{1}, p_{2}\right)$ & View 1 & View 2 \\
\hline Brain $(11505,229,44,144)$ & L-Measure & Tree \\
NFL $(4240,354,177,177)$ & Home & Away \\
Solubility $(1267,228,208,20)$ & Chemistry & Property \\
WebKB $(1051,868,367,501)$ & Link & Text \\
\hline
\end{tabular}

WebKB data set (Sun, 2013). All results will be reproducible upon publication.

Results in Figure 4.3 were based on 50 randomly selected labeled sets at each labeled percentage $100|L| / n_{1}=10,15,20,25,30 \%$, where $n_{1}=n$ for the solubility and WebKB data and $n_{1}=1500$ for the brain and NFL data. Furthermore, 2000 randomly selected unlabeled cases were used to train competitors Co-FTF (SVM) and Man_Co on the brain and NFL data sets to make these techniques computationally feasible. For the regression data sets, a scaled root mean squared error for the unlabeled data $\left(\operatorname{RMSE}_{U} / \hat{\sigma}\right)$ was the performance metric, where $\operatorname{RMSE}_{U}$ is the unlabeled root mean squared error and $\hat{\sigma}$ is the standard error of $\boldsymbol{Y}_{L}$. Unlabeled classification error rate was used for the WebKB data having a binary response. The proposed AGRAPH_MV approach dominates its competitors on these four examples and empirically validates its adoption for wide-ranging, practical, prediction challenges.

\subsection{Discussion}

A multi-view learning approach called AGRAPH_MV was proposed. An important theoretical result motivated AGRAPH_MV by proving an advantage due to joint optimization in noisy learning problems where the true function is small on extrapolations within manifolds. These type of situations are not ideal for existing approaches principled on view agreement or an $\mathrm{s}^{2}$-smoothness assumption. On the other hand, AGRAPH $\_$MV had additional properties that accounted for key trade-offs encountered in practical multi-view learning problems. First, its parameter $\gamma$ regulated a trade-off of $\mathrm{s}^{2}$-smoothness with $\mathrm{s}^{2}$-shrinking. Anchor graphs 
allowed the development of a computationally efficient tool for the detection of manifolds within views. The no-view-distinction term, $\boldsymbol{X} \boldsymbol{\beta}$, added flexibility to trade-off the need to make view distinctions by identifying manifolds within views against using a global regression function. Putting these together, AGRAPH_MV was able to detect manifolds, judge relative view importance with respect to prediction error, shrink the predictions of extreme unlabeled observations away from labeled data on a particular manifold (to reduce variance), and estimate trends in the response within a manifold.

There are several future directions. The computational burden of increased $p$ for the pro-

posed approach is to be mitigated by other $\boldsymbol{\beta}$ regularizers in optimization (4.9). Betweenand within-view variable selection is another interesting problem. The use of sparse graphs by AGRAPH_MV provides an important step towards making these future directions realistic in large data applications. This work justified AGRAPH_MV as a computationally and empirically grounded approach for challenging real-data applications.

\section{Acknowledgements}

The work of Mark Vere Culp was supported in part by the NSF CAREER/DMS-1255045 grant. The opinions and views expressed in this paper are those of the authors and do not reflect the opinions or views at the NSF. 


\section{Appendix: Proofs of Main Theorems}

\section{Proof of Theorem 2}

Let $\widetilde{\boldsymbol{f}}_{U}=-\left(\boldsymbol{\Delta}_{U U}+\frac{p_{\gamma}}{\lambda} \boldsymbol{I}\right)^{-1} \boldsymbol{\Delta}_{U L} \widehat{\boldsymbol{f}}_{0 L}=\sum_{i=1}^{n-m} \frac{\widehat{a}_{0 i} \boldsymbol{o}_{i}}{d_{i}+p_{\gamma}}$. The unlabeled error simplifies to

$$
\begin{aligned}
Q_{1}(\gamma) & \equiv \mathbb{E}\left[\left\|\widehat{\boldsymbol{f}}_{U}-\boldsymbol{m}_{U}\right\|_{2}^{2}\right] \\
& =\mathbb{E}\left[\left\|\widetilde{\boldsymbol{f}}_{U}-\boldsymbol{m}_{U}\right\|_{2}^{2}\right]+2 \mathbb{E}\left[\left(\widetilde{\boldsymbol{f}}_{U}-\boldsymbol{m}_{U}\right)^{T}\left(\widehat{\boldsymbol{f}}_{U}-\widetilde{\boldsymbol{f}}_{U}\right)\right]+\mathbb{E}\left[\left\|\widetilde{\boldsymbol{f}}_{U}-\widehat{\boldsymbol{f}}_{U}\right\|_{2}^{2}\right] \\
& =\mathbb{E}\left[\left\|\widetilde{\boldsymbol{f}}_{U}-\boldsymbol{m}_{U}\right\|_{2}^{2}\right]+\sum_{i=1}^{n-m} \frac{\mathbb{E}\left[\left(\widehat{a}_{0 i}-\widehat{a}_{\gamma i}\right)^{2}\right]}{\left(d_{i}+p_{\gamma}\right)^{2}}+2 \sum_{i=1}^{n-m} \mathbb{E}\left[\left(\frac{\widehat{a}_{0 i}}{d_{i}+p_{\gamma}}-b_{i}\right) \frac{\left(\widehat{a}_{0 i}-\widehat{a}_{\gamma i}\right)}{d_{i}+p_{\gamma}}\right] \\
& \leq \mathbb{E}\left[\left\|\widetilde{\boldsymbol{f}}_{U}-\boldsymbol{m}_{U}\right\|_{2}^{2}\right]+\varepsilon^{2}+\frac{2 \varepsilon}{\sqrt{n-m}} \sum_{i=1}^{n-m} \mathbb{E}\left[\left|\frac{\widehat{a}_{0 i}}{d_{i}+p_{\gamma}}-b_{i}\right|\right] \\
& \leq \mathbb{E}\left[\left\|\widetilde{\boldsymbol{f}}_{U}-\boldsymbol{m}_{U}\right\|_{2}^{2}\right]+\frac{2 M}{\sqrt{n-m}} \boldsymbol{\varepsilon}+\boldsymbol{\varepsilon}^{2} .
\end{aligned}
$$

From Assumption 2.2, let $r_{i}=\boldsymbol{o}_{i}^{T} \boldsymbol{\Sigma} \boldsymbol{o}_{i}$, so

$$
\begin{aligned}
Q_{2}\left(p_{\gamma}\right) & \equiv \mathbb{E}\left[\left\|\widetilde{\boldsymbol{f}}_{U}-\boldsymbol{m}_{U}\right\|_{2}^{2}\right]=\sum_{i=1}^{n-m} \mathbb{E}\left[\left(\frac{\widehat{a}_{0 i}}{d_{i}+p_{\gamma}}-b_{i}\right)^{2}\right] \\
& =\sum_{i=1}^{n-m} \frac{r_{i} \sigma^{2}+\left(a_{i}-b_{i}\left(d_{i}+p_{\gamma}\right)\right)^{2}}{\left(d_{i}+p_{\gamma}\right)^{2}} .
\end{aligned}
$$

The derivative of 4.15) w.r.t. $p_{\gamma}$ evaluated at $p_{\gamma}=0$ is

$$
\begin{aligned}
\left.\frac{\partial Q_{2}\left(p_{\gamma}\right)}{\partial p_{\gamma}}\right|_{p_{\gamma}=0} & =-\left.2 \sum_{i=1}^{n-m}\left(\frac{r_{i} \sigma^{2}+\left(a_{i}-b_{i}\left(d_{i}+p_{\gamma}\right)\right)^{2}}{\left(d_{i}+p_{\gamma}\right)^{3}}+\frac{\left(b_{i}\left(a_{i}-b_{i}\left(d_{i}+p_{\gamma}\right)\right)\right)}{\left(d_{i}+p_{\gamma}\right)^{2}}\right)\right|_{p_{\gamma}=0} \\
& =-2 \sum_{i=1}^{n-m} \frac{r_{i} \sigma^{2}+a_{i}^{2}-a_{i} b_{i} d_{i}}{d_{i}^{3}}
\end{aligned}
$$

and is negative if (4.8) holds. 


\section{Proof of Theorem 3}

If $\widetilde{\boldsymbol{w}}_{j}=\boldsymbol{R}^{1 / 2} \boldsymbol{w}_{j}$, then $\left\{\tau_{j}, \widetilde{\boldsymbol{w}}_{j}\right\}$ is an eigen-decomposition of $\boldsymbol{R}^{-1 / 2}{ }^{T} \boldsymbol{T}_{U}^{T} \boldsymbol{T}_{U} \boldsymbol{R}^{-1 / 2} \succeq 0$ such that

$$
\begin{aligned}
\boldsymbol{w}_{j^{\prime}}^{T} \boldsymbol{T}_{U}^{T} \boldsymbol{T}_{U} \boldsymbol{w}_{j} & =\widetilde{\boldsymbol{w}}_{j^{\prime}}^{T} \boldsymbol{R}^{-1 / 2^{T}} \boldsymbol{T}_{U}^{T} \boldsymbol{T}_{U} \boldsymbol{R}^{-1 / 2} \widetilde{\boldsymbol{w}}_{j} \\
& =\tau_{j} \widetilde{\boldsymbol{w}}_{j^{\prime}}^{T} \widetilde{\boldsymbol{w}}_{j}=\tau_{j} \mathscr{I}_{\left\{j^{\prime}=j\right\}} .
\end{aligned}
$$

Let $\widetilde{\boldsymbol{c}}=R^{1 / 2} \widehat{\boldsymbol{c}}$. Project $\widetilde{\boldsymbol{c}}=\sum_{j=1}^{\widetilde{p}} \widehat{a}_{j} \widetilde{\boldsymbol{w}}_{j}$, so $\widehat{\boldsymbol{c}}=\sum_{j=1}^{\widetilde{p}} \widehat{a}_{j} \boldsymbol{w}_{j}$ and $\widehat{\boldsymbol{\psi}}=\sum_{j=1}^{\widetilde{p}} \frac{\widehat{a}_{j}}{1+p_{\gamma} \tau_{j}} \boldsymbol{w}_{j}$. Let $r_{j}=$ $\boldsymbol{w}_{j}^{T} \widetilde{\boldsymbol{\Sigma}} \boldsymbol{w}_{j}$. Then 4.16 results in

$$
\begin{aligned}
Q_{3}\left(p_{\gamma}\right) & \equiv \mathbb{E}\left[\left\|\boldsymbol{T}_{U}\left(\widehat{\boldsymbol{\psi}}_{\gamma}-\boldsymbol{\theta}\right)\right\|_{2}^{2}\right] \\
& =\sum_{j=1}^{\widetilde{p}} \tau_{j} \mathbb{E}\left[\left(\frac{\widehat{a}_{j}}{1+p_{\gamma} \tau_{j}}-q_{j}\right)^{2}\right] \\
& =\sum_{j=1}^{\widetilde{p}} \frac{\tau_{j}}{\left(1+p_{\gamma} \tau_{j}\right)^{2}}\left(r_{j} \sigma^{2}+h_{j}\left(p_{\gamma}\right)\right),
\end{aligned}
$$

where $h_{j}\left(p_{\gamma}\right)=\left(\mu_{j}-q_{j}\left(1+p_{\gamma} \tau_{j}\right)\right)^{2}$. Taking the derivate of 4.17) with respect to $p_{\gamma}$ yields

$$
\left.\frac{\partial Q_{3}\left(p_{\gamma}\right)}{\partial p_{\gamma}}\right|_{p_{\gamma}=0}=\sum_{j=1}^{\tilde{p}}-2 \tau_{j}^{2}\left(r_{j} \sigma^{2}+\mu_{j}^{2}-\mu_{j} q_{j}\right),
$$

which is negative if $\sigma^{2}$ satisfies the given bound. 


\section{Chapter 5}

\section{Discussion and Future Directions}

\subsection{General Discussion}

Semi-supervised optimization frameworks based on labeled loss and joint training were explored for the graph-based setting. Our ideas were motivated from a theoretical perspective to solve practical data problems and were then followed-up by an extensive empirical analysis

providing clear dominance over the state-of-the-art. All results were based on CV. We beat these existing techniques at their best. This effort culminate into two new semi-supervised approaches S4PM and AGRAPH_MV that are novel, useful, and computationally efficient.

In Section 2.1, the problem was carefully outlined and motivated. Several optimization problems in the literature were described. Section 2.2 provided a brief history of the semisupervised learning problem from early techniques to the much more recent graph-based approaches. Motivation involving various manifold assumptions for incorporate proximity graphs in semi-supervised learning was provided in Section 2.2.1. Section 2.2.3 illustrated the problems associated with graph-based learning which motivated pursuant Section 2.3 The anchor graph technique discussed in detail in Section 2.3 mitigates the high computational burdens of graph-based semi-supervised learning by reducing the computational com- 
plexity, but it suffers from a vital drawback. The performance with respect to the accuracy of the status quo is reduced drastically, and the anchor graph method is outperformed by almost every machine learning technique tried. The research work in this dissertation is stimulated by creating a competitive graph-based semi-supervised methodology that retains the speed of these techniques with improved prediction performance.

The first major contribution of this thesis is the proposed safe semi-parametric semisupervised model (S4PM) in Section 3. This approach moves away from the strong smoothness assumptions required for existing semi-supervised methodologies yielding a more robust fit to real data problems. Moreover, this safe graph-based technique is computationally efficient with minimal learning time for large data problems. Empirical results show that the S4PM method is computationally efficient and achieves competitive accuracies that outperform the state-of-the-art techniques in both regression and classification. These are the same settings where the original anchor graph method failed.

A multi-view semi-supervised shrinking $\left(s^{2}\right.$-shrinking) based learning method was proposed in Section 4 which jointly learns on several views and improvements were proven for some realistic noisy data problems. The AGARPH_MV enhanced the learning mechanism by amending manifold detection, view importance, and prediction shrinking into a single unified framework. Thorough rationales behind the improvement due to AGRAPH_MV were also presented. Furthermore, the computational complexity of this method is less than that of existing multi-view techniques.

\subsection{Future Research Directions}

There are several research questions to be pursued. Parallel processing is an integral component for improving the efficiency of computational approaches especially for high dimensional data problems. Several tools for parallelization are readily available including R pack- 
ages snow, snowfall, parallel, foreach, Hadoop, and Apache Spark. The anchor graph construction mechanism utilized in this research is an embarrassingly parallel process and can be distributed among several nodes in any parallel framework. One future goal is to convert the entire process into a parallel implementation and prepare a $R$ package for wide-spread use.

Chapter 3 presents the S4PM. A natural extension is to generalize its optimization to penalized regression problems under Hilbert space $\mathscr{H}_{K}$ optimization which builds on the definitions given in Section 2.1. This direction ultimately requires extending the joint optimization into the Hilbert space setting with

$$
\min _{\boldsymbol{Y}_{U}, \beta \in \mathbb{R}^{m}, \boldsymbol{f} \in \mathscr{H}_{K}}\left\{L\left(\boldsymbol{Y}\left(\boldsymbol{Y}_{U}\right), \boldsymbol{Z}_{L} \beta+\boldsymbol{f}(\boldsymbol{x})\right)+\lambda \beta^{T} \tilde{\boldsymbol{\Delta}} \beta+\|\boldsymbol{f}\|_{\mathscr{H}_{K}}^{2}+\gamma \mathbb{P}\left(\boldsymbol{Y}_{U}\right)\right\}
$$

where the space for $Y_{U}$ provides an interesting challenge to be investigated. Fitting this technique with linear, polynomial and the RBF kernels of (2.13) are to be examined. This entire extension is expected to yield fruitful improvements.

Section 4 demonstrated the incorporation of multiple views of data with improved accuracy into a single model. Though AGRAPH_MV is computationally efficient, learning from multiple views with a large number of variables is a daunting task and increases the computational burden of any graph-based approach. Thus another, more practical research direction is the variable selection problem within and between views. 


\section{Appendix A}

\section{Investigation of Tuning Parameters}

The anchor graph procedure described in section 2.3 relies heavily on several tuning parameters, the number of anchor points $m$, the number of closest anchors to an observation $s$ and a threshold parameter $c_{n}$ of LAE algorithm. This section concentrates on the choice of the tuning parameters and their effects on the anchor graph algorithm. Two datasets are chosen for this task: (1) Meatspec data and (2) Power data.

\section{A.1 Investigation on the Anchor Points}

Section 2.3.1 described the anchor points and the role of properly chosen anchor points on the entire outcome of the algorithm. In this section, three separate clustering mechanisms are implemented in order to choose an adequate set of anchor points. The clustering algorithms are (1) K-Means clustering, (2) Hierarchical clustering, and (3) Fuzzy C-Means clustering. Three different number of anchors $m$ are chosen for each dataset and the means, and standard deviations in each case are reported. It should be noted that for a dataset with a large number of observation $(n>1667)$, the maximum number of anchors are chosen as 500. Since larger choices of $m$ will incur more instability and additional computation time which diminishes 
Table A.1: Anchor Graph Based Prediction Analysis with the Meatspec Data. The Number of Anchors are 30, 50, and 70 Respectively.

\begin{tabular}{|c|c|c|c|c|c|c|c|c|c|}
\hline \multirow{2}{*}{ Error Rate } & \multicolumn{3}{|c|}{ K-Means Anchors } & \multicolumn{3}{c|}{ HClust Anchors } & \multicolumn{3}{c|}{ C-Means Anchors } \\
\cline { 2 - 10 } & 30 & 50 & 70 & 30 & 50 & 70 & 30 & 50 & 70 \\
\hline \hline Mean of error & 0.071 & 0.068 & 0.066 & 0.208 & 0.209 & 0.208 & 0.069 & 0.071 & 0.070 \\
\hline SD of error & 0.020 & 0.021 & 0.018 & 0.030 & 0.030 & 0.030 & 0.018 & 0.019 & 0.018 \\
\hline
\end{tabular}

Table A.2: Anchor Graph Based Prediction Analysis with the Power Data Taking the Number of Anchors as 100, 250 and 500 Respectively.

\begin{tabular}{|c|c|c|c|c|c|c|c|c|c|}
\hline \multirow{2}{*}{ Error Rate } & \multicolumn{3}{|c|}{ K-Means Anchors } & \multicolumn{3}{c|}{ HClust Anchors } & \multicolumn{3}{c|}{ C-Means Anchors } \\
\cline { 2 - 10 } & 100 & 250 & 500 & 100 & 250 & 500 & 100 & 250 & 500 \\
\hline \hline Mean of error & 0.015 & 0.014 & 0.013 & 0.032 & 0.032 & 0.032 & 0.109 & 0.109 & 0.108 \\
\hline SD of error & $<0.01$ & $<0.01$ & $<0.01$ & $<0.01$ & $<0.01$ & $<0.01$ & 0.013 & 0.012 & 0.012 \\
\hline
\end{tabular}

the overall performance of the anchor graph technique both in accuracy and speed. The number of labeled cases for each run is 50 for the Meatspec data and 1000 for the Power dataset, and these experiments were repeated 20 times.

It is evident from Table A.1 and Table A.2 that the anchor points chosen via K-Means clustering mechanism yields better performance than other clustering techniques investigated here. Moreover, the anchor graph algorithm with 'large enough' anchors performs fairly well when compared with smaller number counterparts. Clearly the choice of 'large enough' number of anchors prevails, and our choice is empirically justified.

\section{A.2 Investigation on the Number of Anchors ' $s$ '}

Each observation in anchor graph technique is mapped to its nearest $s$ anchors. Therefore the choice of $s$ is critical to the entire graph construction process (same as $k$ in $\mathrm{K}-\mathrm{NN}$ described in Section 2.1. Smaller $s$ values tend to not have enough connectivity information in the graph, whereas larger values tend to gives more noisy graphs. Analysis on the Meatspec and Power data are given below with choices of nearest anchors from 2 to 10 and same number 
of labeled cases as Section A.1.

Table A.3: The Mean and Variance of Prediction Accuracies for the Meatspec Data

\begin{tabular}{|c|c|c|c|c|c|c|c|c|c|}
\hline \multirow{2}{*}{ Error Rate } & \multicolumn{9}{|c|}{ Choice of } \\
\cline { 2 - 11 } & 2 & 3 & 4 & 5 & 6 & 7 & 8 & 9 & 10 \\
\hline \hline Mean of error & 0.0618 & 0.0639 & 0.0644 & 0.0615 & 0.0616 & 0.0653 & 0.0630 & 0.0657 & 0.0638 \\
\hline SD of error & 0.016 & 0.021 & 0.020 & 0.016 & 0.016 & 0.017 & 0.021 & 0.016 & 0.015 \\
\hline
\end{tabular}

Table A.4: The Mean and Variance of Prediction Accuracies for the Power Data

\begin{tabular}{|c|c|c|c|c|c|c|c|c|c|}
\hline \multirow{2}{*}{ Error Rate } & \multicolumn{10}{|c|}{ Choice of $s$} \\
\cline { 2 - 10 } & 2 & 3 & 4 & 5 & 6 & 7 & 8 & 9 & 10 \\
\hline \hline Mean of error & 0.0139 & 0.0139 & 0.0139 & 0.0138 & 0.0138 & 0.0140 & 0.0140 & 0.0141 & 0.0141 \\
\hline SD of error & $<0.01$ & $<0.01$ & $<0.01$ & $<0.01$ & $<0.01$ & $<0.01$ & $<0.01$ & $<0.01$ & $<0.01$ \\
\hline
\end{tabular}

Tables A.3 and A.4 shows that the anchor graph with 5 nearest anchors performs better than other choices of $s$ which helps justify our usage of this value throughout the dissertation.

\section{A.3 Investigation on the LAE Threshold ' $c_{n}$ '}

The final parameter to be optimized is the threshold parameter that controls the optimum number of evaluation of the LAE algorithm described in Section 2.3.2. Utilizing the Meatspec and Power dataset we investigate several choices of threshold parameter $c_{n}$ ranging from 2 iterations to maximum 10 iterations. The results shown in Table A.5 and A.6 shows the average error rate and error dispersion with other parameters fixed to their CV-optimum. The observations indicate no clear impact of the choice of threshold parameter $c_{n}$ on the entire anchor graph method. Thus the parameter value is kept at a smaller value 4 for the other analysis. 
Table A.5: The Mean and Variance of Errors with Different Evaluation of LAE Algorithm int he Meatspec Data.

\begin{tabular}{|c|c|c|c|c|c|c|c|c|c|}
\hline \multirow{2}{*}{ Error Rate } & \multicolumn{9}{|c|}{ Choice of $c_{n}$} \\
\cline { 2 - 11 } & 2 & 3 & 4 & 5 & 6 & 7 & 8 & 9 & 10 \\
\hline \hline Median of error & 0.0616 & 0.0616 & 0.0616 & 0.0616 & 0.0616 & 0.0616 & 0.0616 & 0.0617 & 0.0617 \\
\hline SD of error & 0.021 & 0.021 & 0.021 & 0.021 & 0.021 & 0.021 & 0.020 & 0.020 & 0.020 \\
\hline
\end{tabular}

Table A.6: The Mean and Variance of Errors with Different Evaluation of LAE Algorithm in the Power Data.

\begin{tabular}{|c|c|c|c|c|c|c|c|c|c|}
\hline \multirow{2}{*}{ Error Rate } & \multicolumn{9}{|c|}{ Choice of $c_{n}$} \\
\cline { 2 - 11 } & 2 & 3 & 4 & 5 & 6 & 7 & 8 & 9 & 10 \\
\hline \hline Mean of error & 0.0139 & 0.0139 & 0.0139 & 0.0139 & 0.0139 & 0.0139 & 0.0139 & 0.0139 & 0.0138 \\
\hline SD of error & $<0.01$ & $<0.01$ & $<0.01$ & $<0.01$ & $<0.01$ & $<0.01$ & $<0.01$ & $<0.01$ & $<0.01$ \\
\hline
\end{tabular}




\section{Bibliography}

Agarwala. Probability of error of some adaptive pattern-recognition machines. IEEE Transactions on Information Theory, 11:363-371, 1970.

G Ascoli, D Donohue, and M Halavi. NeuroMorpho.Org: A central resource for neuronal morphologies. The Journal of Neuroscience, 27(35):9247-9251, 2007.

M Azizyan, A Singh, and L Wasserman. Density-sensitive semisupervised inference. The Annals of Statistics, 41(2):751-771, 2013.

FR Bach. Consistency of the group lasso and multiple kernel learning. Journal of Machine Learning Research, 9:1179-1225, 2008.

M Belkin, P Niyogi, and V Sindhwani. Manifold regularization: a geometric framework for learning from labeled and unlabeled examples. Journal of Machine Learning Research, 7: 2399-2434, 2006.

AW Bowman. An alternative method of cross-validation for the smoothing of density estimates. Biometrika, 71(2):353-360, 1984.

MW Browne, R Cudeck, KA Bollen, and JS Long. Alternative ways of assessing model fit. Sage Focus Editions, 154:136-136, 1993.

A Buja, T Hastie, and R Tibshirani. Linear smoothers and additive models. The Annals of Statistics, 17:453-510, 1989. 
O Chapelle, M Chi, and A Zien. A continuation method for semi-supervised SVMs. In Proceedings of the 23rd International Conference on Machine Learning, pages 185-192, New York, NY, USA, 2006a. ACM.

O Chapelle, M Chi, and A Zien. A continuation method for semi-supervised svms. In Proceedings of the 23rd international conference on Machine learning, pages 185-192. ACM, 2006b.

O Chapelle, B Schölkopf, and A Zien, editors. Semi-supervised learning. MIT Press, Cambridge, MA, 2006c. URL http://www.kyb.tuebingen.mpg.de/ssl-book.

O Chapelle, V Sindhwani, and S Keerthi. Optimization techniques for semi-supervised support vector machines. Journal of Machine Learning Research, 9:203-233, 2008.

DB Cooper and JH Freeman. On the asymptotic improvement in the out-come of supervised learning provided by additional nonsupervised learning. Computers, IEEE Transactions on, 100(11):1055-1063, 1970.

M Culp. On propagated scoring for semisupervised additive models. Journal of the American Statistical Association, 106(493):248-259, 2011a.

M Culp. spa: A semi-supervised $\mathrm{r}$ package for semi-parametric graph-based estimation. Journal of Statistical Software, 40(10):1-29, 2011 b.

M Culp and G Michailidis. Graph-based semisupervised learning. Pattern Analysis and Machine Intelligence, IEEE Transactions on, 30(1):174-179, 2008.

M Culp, G Michailidis, and K Johnson. On multi-view learning with additive models. Annals of Applied Statistics, 3(1):292-318, 2009.

M Culp, K Ryan, and P Banerjee. On safe semi-supervised learning. IEEE Pattern Analysis and Machine Intelligence (submitted), 2015. 
MV Culp. On propagated scoring for semisupervised additive models. Journal of the American Statistical Association, 106(493):248-259, 2011c.

MV Culp and G Michailidis. A co-training algorithm for multi-view data with applications in data fusion. Journal of Chemometrics, 23:294-303, 2009.

MV Culp and KJ Ryan. Joint harmonic functions and their supervised connections. Journal of Machine Learning Research, 14:3721-3752, 2013.

H Cuntz, F Forstner, A Borst, and M Häusser. One rule to grow them all: A general theory of neuronal branching and its practical application. PLoS Computational Biology, 8(6), 2010.

AP Dempster, NM Laird, and DB Rubin. Maximum likelihood from incomplete data via the em algorithm. Journal of the royal statistical society. Series B (methodological), pages $1-38,1977$.

L Deng, J Li, JT Huang, K Yao, D Yu, F Seide, M Seltzer, G Zweig, X He, J Williams, et al. Recent advances in deep learning for speech research at microsoft. In Acoustics, Speech and Signal Processing (ICASSP), 2013 IEEE International Conference on, pages 8604-8608. IEEE, 2013.

B Efron. Estimating the error rate of a prediction rule: improvement on cross-validation. Journal of the American Statistical Association, 78(382):316-331, 1983.

B Everitt and T Hothorn. An introduction to applied multivariate analysis with $R$. Springer Science \& Business Media, 2011.

J Faraway. faraway: Functions and Datasets for Books by Julian Faraway, 2016. URL https: / /CRAN.R-project.org/package=faraway, R package version 1.0.7. 
M Fernández-Delgado, E Cernadas, S Barro, and D Amorim. Do we need hundreds of classifiers to solve real world classification problems? Journal of Machine Learning Research, 15:3133-3181, 2014.

J Friedman, T Hastie, and R Tibshirani. Regularization paths for generalized linear models via coordinate descent. Journal of Statistical Software, 33(1):1-22, 2010.

T Hastie, R Tibshirani, and J Friedman, editors. The Elements of Statistical Learning (Data Mining, Inference and Prediction, Second Edition). Springer, New York, NY, 2009.

G Heckman. Harmonic analysis and special functions on symmetric spaces. Academic Press, 1995.

M Hein, J Audibert, and UV Luxburg. From graphs to manifolds-weak and strong pointwise consistency of graph Laplacians. In Proceedings of the 18th Annual Conference on Learning Theory, pages 470-485, New York, NY, USA, 2005. Springer.

DW Hosmer Jr. A comparison of iterative maximum likelihood estimates of the parameters of a mixture of two normal distributions under three different types of sample. Biometrics, pages $761-770,1973$.

AK Jain, A Ross, and S Prabhakar. An introduction to biometric recognition. IEEE Transactions on Circuits and Systems for Video Technology, 14(1):4-20, 2004.

J Ji, F Shao, R Sun, N Zhang, and G Liu. A TSVM based semi-supervised approach to SAR image segmentation. In Education Technology and Training, 2008. and 2008 International Workshop on Geoscience and Remote Sensing. ETT and GRS 2008. International Workshop on, volume 1, pages 495-498. IEEE, 2008.

T Joachims. Transductive inference for text classification using support vector machines. In ICML, volume 99, pages 200-209, 1999. 
A Karatzoglou, A Smola, K Hornik, and A Zeileis. kern lab - an S4 package for kernel methods in R. Journal of Statistical Software, 11(9):1-20, 2004. URL http: / /www . jstatsoft.org/v11/i09/

M Karlen, J Weston, A Erkan, and R Collobert. Large scale manifold transduction. In Proceedings of the 25th international conference on Machine learning, pages 448-455. ACM, 2008.

M Kawakita and T Jun'ichi. Safe semi-supervised learning based on weighted likelihood. Neural Networks, 53(1):146-164, 2014.

M Kuhn. Building predictive models in R using the caret package. Journal of Statistical Software, 28(5):1-26, 2014.

M Kuhn and K Johnson, editors. Applied Predictive Modeling. Springer, New York, NY, 2013.

SY Kung, MW Mak, and SH Lin. Biometric authentication: A machine learning approach. Prentice Hall Professional Technical Reference Upper Saddle River, 2005.

J Lafferty and L Wasserman. Statistical analysis of semi-supervised regression. In J.C. Platt, D. Koller, Y. Singer, and S.T. Roweis, editors, Advances in Neural Information Processing Systems 20, pages 801-808. Curran Associates, Inc., 2008.

Z Lao, D Shen, Z Xue, B Karacali, SM Resnick, and C Davatzikos. Morphological classification of brains via high-dimensional shape transformations and machine learning methods. Neuroimage, 21(1):46-57, 2004.

Y Li and Z Zhou. Towards making unlabeled data never hurt. In Proceedings of the $28^{\text {th }}$ International Conference on Machine Learning, pages 1081-1088, New York, NY, USA, 2011. ACM. 
YF Li, JT Kwok, and ZH Zhou. Cost-sensitive semi-supervised support vector machine. In Proceedings of the National Conference on Artificial Intelligence, volume 1, page 500, 2010.

M Lichman. UCI machine learning repository, 2013. URL http://archive.ics . uci.edu/ml.

W Liu, J He, and S Chang. Large graph construction for scalable semi-supervised learning. In Proceedings of the 27rd International Conference on Machine Learning, pages 679687, Haifa, Israel, 2010. ACM.

W Liu, C Mu, S Kumar, and S Chang. Discrete graph hashing. In Z. Ghahramani, M. Welling, C. Cortes, N. Lawrence, and K.Q. Weinberger, editors, Advances in Neural Information Processing Systems 27, pages 3419-3427. Curran Associates, Inc., 2014.

GJ McLachlan and S Ganesalingam. Updating a discriminant function on the basis of unclassified data. Communications in Statistics-Simulation and Computation, 11(6):753-767, 1982.

Y Nesterov. Smooth minimization of non-smooth functions. Mathematical programming, 103(1):127-152, 2005.

L Page, S Brin, R Motwani, and T Winograd. The PageRank citation ranking: bringing order to the web. Stanford InfoLab, 1999.

R Palaniappan and DP Mandic. Biometrics from brain electrical activity: A machine learning approach. IEEE Transactions on Pattern Analysis and Machine Intelligence, 29(4):738$742,2007$.

P Pudil and J Novovičová. Novel methods for feature subset selection with respect to 
problem knowledge. In Feature Extraction, Construction and Selection, pages 101-116. Springer, 1998.

J Ratsaby and SS Venkatesh. Learning from a mixture of labeled and unlabeled examples with parametric side information. In Proceedings of the eighth annual conference on Computational learning theory, pages 412-417. ACM, 1995.

J Richiardi, S Achard, H Bunke, and D Van De Ville. Machine learning with brain graphs: predictive modeling approaches for functional imaging in systems neuroscience. Signal Processing Magazine, IEEE, 30(3):58-70, 2013.

KJ Ryan and MV Culp. On semi-supervised linear regression in covariate shift problems. Journal of Machine Learning Research, In Press., 2015.

HJ Scudder. Probability of error of some adaptive pattern-recognition machines. IEEE Transactions on Information Theory, 11:363-371, 1965.

F Sebastiani. Machine learning in automated text categorization. ACM computing surveys (CSUR), 34(1):1-47, 2002.

V Sindhwani and DS Rosenberg. An RKHS for multi-view learning and manifold coregularization. In Proceedings of the 25th International Conference on Machine Learning, pages 976-983, New York, NY, 2008. ACM.

A Singh, R Nowak, and X Zhu. Unlabeled data: Now it helps, now it doesn't. In D. Koller, D. Schuurmans, Y. Bengio, and L. Bottou, editors, Advances in Neural Information Processing Systems 21, pages 1513-1520. Curran Associates, Inc., 2009.

N Sokolovska, O Cappé, and F Yvon. The asymptotics of semi-supervised learning in discriminative probabilistic models. In Proceedings of the 25th international conference on Machine learning, pages 984-991. ACM, 2008. 
N Subrahmanya and YC Shin. Sparse multiple kernel learning for signal processing applications. IEEE Transactions on Pattern and Machine Learning, 32:788-798, 2010.

S Sun. A survey of multi-view machine learning. Neural Computing and Applications, 23 (7):2031-2038, 2013.

R Core Team. R: A Language and Environment for Statistical Computing. R Foundation for Statistical Computing, Vienna, Austria, 2015. URL https : / /www.R-project. $\operatorname{org} /$.

RC Tryon. Cluster analysis: correlation profile and orthometric (factor) analysis for the isolation of unities in mind and personality. Edwards brother, Incorporated, lithoprinters and publishers, 1939.

VN Vapnik and AY Chervonenkis. Theory of Pattern Recognition [in Russian]. Nauka, USSR, 1974.

J Wang, T Jebara, and S Chang. Semi-supervised learning using greedy max-cut. Journal of Machine Learning Research, 14:771-800, 2013.

W Wang and Z Zhou. A new analysis of co-training. In Proceedings of the 27rd International Conference on Machine Learning, pages 1135-1142, Haifa, Israel, 2010. ACM.

Y Wang and S Chen. Safety-aware semi-supervised classification. IEEE Transactions on Neural Networks and Learning Systems, 24(11):1763-1772, 2013.

D Zhou, O Bousquet, TN Lal, J Weston, and B Schölkopf. Learning with local and global consistency. In S. Thrun, L.K. Saul, and B. Schölkopf, editors, Advances in Neural Information Processing Systems 16, pages 321-328. MIT Press, 2004a.

D Zhou, O Bousquet, TN Lal, J Weston, and B Schölkopf. Learning with local and global consistency. Advances in neural information processing systems, 16(16):321-328, 2004b. 
X Zhu, Z Ghahramani, J Lafferty, et al. Semi-supervised learning using gaussian fields and harmonic functions. In ICML, volume 3, pages 912-919, 2003. 